\title{
NUMBERING OF FULLERENES
}

\section{(IUPAC Recommendations 2005)}

\author{
F. COZZI ${ }^{1}$, W. H. POWELL ${ }^{2, \ddagger}$, AND C. THILGEN ${ }^{3}$ \\ ${ }^{1}$ Dipartimento di Chimica Organica e Industriale via Golgi, 19-I-20133, Milano, Italy; ${ }^{2} 1436$ \\ Havencrest Ct., Columbus, OH 43220, USA; ${ }^{3}$ Laboratorium für Organische Chemie - ETH Zürich, \\ Wolfgang-Pauli-Strasse 10, CH-8093, Zürich, Switzerland
}

*Membership of the Division Committee during preparation of this report (2002-2004) was as follows:

President: A. D. McNaught (UK, 2002-2005); Vice-President: G. P. Moss (UK, 2004-2005); Secretary: W. H. Powell (USA, 2002-2005); Titular Members: T. Damhus (Denmark, 2004-2007); M. Dennis (USA, 2002-2003); R. M. Hartshorn (New Zealand, 2004-2007); M. Hess (Germany, 2002-2003); H. D. Kaesz (USA, 2002-2005); J. Kahovec (Czech Republic, 2004-2007); G. J. Leigh (UK, 2002-2003); G. P. Moss (UK, 2002-2003); J. Nyitrai (Hungary, 2004-2007); W. G. Town (UK, 2002-2003); A. Williams (USA, 2002-2005); A. Yerin (Russia, 2004-2007); Associate Members: J. Brecher (USA, 2004-2005); S. R. Heller (USA, 2002-2005); M. Hess (Germany, 2004-2005); A. J. Lawson (Germany, 2002-2005); G. J. Leigh (UK, 2004-2005); B. M. Novak (USA, 2002-2003); M. J. Toussant (USA, 2004-2005).

¥Corresponding author: E-mail: wpowell2@juno.com

Republication or reproduction of this report or its storage and/or dissemination by electronic means is permitted without the need for formal IUPAC permission on condition that an acknowledgment, with full reference to the source, along with use of the copyright symbol $\odot$, the name IUPAC, and the year of publication, are prominently visible. Publication of a translation into another language is subject to the additional condition of prior approval from the relevant IUPAC National Adhering Organization. 


\title{
Numbering of fullerenes
}

\section{(IUPAC Recommendations 2005)}

\begin{abstract}
Rules for numbering $\left(\mathrm{C}_{60}-I_{\mathrm{h}}\right)[5,6]$ fullerene and $\left(\mathrm{C}_{70}-D_{5 \mathrm{~h}(6)}\right)[5,6]$ fullerene were codified in a publication "Nomenclature for the $\left(\mathrm{C}_{60^{-}} \mathrm{I}_{\mathrm{h}}\right)[5,6]$ and $\left(\mathrm{C}_{70}-D_{5 \mathrm{~h}(6)}\right)[5,6]$ fullerenes" published in Pure Appl. Chem. 74 (4), 629-695 (2002). The current publication contains recommendations for numbering a wide variety of fullerenes of different sizes, with rings of different sizes, from $\mathrm{C}_{20}$ to $\mathrm{C}_{120}$, and of various point group symmetries, including low symmetries such as $C_{\mathrm{s}}, C_{\mathrm{i}}$, and $C_{1}$, as well as many fullerenes that have been isolated and well characterized as pristine carbon allotropes or as derivatives. These recommendations are based on the principles established in the earlier publication and aim at the identification of a well-defined and preferably contiguous helical pathway for numbering. Rules for systematically completing the numbering of fullerene structures for which a contiguous numbering pathway becomes discontiguous are presented.
\end{abstract}

Keywords: fullerenes; numbering; recommendations; nomenclature; helical numbering pathway; Division VIII.

\section{INTRODUCTION}

In 1995, a preliminary survey on numbering and nomenclature for fullerenes was published [1]. It described two types of names for fullerenes, one proposed by some workers in the field and another one used by the Chemical Abstracts Service (CAS); and two systems for numbering fullerene skeletons, one proposed in a publication by R. Taylor [2], and one published by CAS [3]. In addition, alternative methods for naming derivatives of fullerenes were discussed. More recently, a document appeared reporting IUPAC recommendations for the nomenclature for the $\mathrm{C}_{60}-I_{\mathrm{h}}$ and $\mathrm{C}_{70}-D_{5 \mathrm{~h}(6)}$ fullerenes and their derivatives [4]. This report was limited to these fullerenes because there are only a small number of known derivatives of only a few other fullerenes.

The present document contains recommendations for the numbering of fullerenes other than $\left(\mathrm{C}_{60} \mathrm{I}_{\mathrm{h}}\right)[5,6]$ fullerene and $\left(\mathrm{C}_{70}-D_{5 \mathrm{~h}(6)}\right)[5,6]$ fullerene. The parenthetical prefix gives the carbon content and the point group symbol; the bracketed numbers indicate the ring sizes in the fullerene. The latter is important in fullerenes with rings other than five- and six-membered. The subscript parenthetical (6) following the point group symbol $D_{5 \mathrm{~h}}$ in the latter name indicates that the five-membered ring on the five-fold symmetry axis is surrounded by six-membered rings. This distinguishes this fullerene from an isomeric $\left(\mathrm{C}_{70}-D_{5 \mathrm{~h}}\right)[5,6]$ fullerene having five-membered rings surrounding the five-membered ring on the five-fold symmetry axis designated by the name $\left(\mathrm{C}_{70}-D_{5 \mathrm{~h}(5)}\right)[5,6]$ fullerene. In the present document, fullerene structures are unambiguously identified by CAS Registry Numbers and the reference numbers of An Atlas of Fullerenes, if available [5].

The recommendations are largely based on those reported in ref. [4], and can in principle be used for numbering all fullerenes. One of the aims pursued with the development of these recommendations was to devise a numbering method that is simpler than the existing CAS procedures [3], that uses symmetry elements as reference entities, and that can be applied "manually" by the chemical public to a structural drawing of a fullerene. It soon became clear that such a method, allowing a relatively easy search for a preferentially contiguous and pretty unambiguous numbering of "small" fullerenes, does not constitute a mathematically robust and absolutely unambiguous numbering procedure for any given 
fullerene structure - an endeavor that would require computational methods. This antagonism is underpinned by the number of possible $\mathrm{C}_{n}$ isomers, which increases overwhelmingly with $n$ : for example, there are 31924 isomers of $\left(\mathrm{C}_{80}\right)$ [5,6]fullerene (enantiomeric pairs being counted as one), and contiguous paths of interconnected vertices cannot be found for all of them [5]. On the other hand, the practicability of the present system was tested successfully on 69 fullerenes (including most of the isolated and structurally characterized carbon cages), and it can be expected to perform well in the entire range of "small" fullerenes. It should finally be mentioned that this method conveniently provides the same numberings as the CAS procedure [3] for $\left(\mathrm{C}_{60}-I_{\mathrm{h}}\right)[5,6]$ fullerene and $\left(\mathrm{C}_{70}-D_{5 \mathrm{~h}(6)}\right)[5,6]$ fullerene, which are the objects of the vast majority of publications on fullerenes.

\section{GENERAL CONSIDERATIONS ON FULLERENE NUMBERING}

The identification of a well-defined and preferably contiguous helical numbering pathway is the cornerstone of fullerene numbering. Such a pathway represents a double-conical helix in the sense that, starting from a given atom (a "pole" of the spheroid), reaches all the other atoms of the fullerene moving in a clockwise or counterclockwise direction through the two "hemispheres" to reach the opposite "pole". (In a Schlegel diagram with the starting point of the numbering located at the center of the representation, the pathway corresponds to an expanding spiral.) The construction of the pathway starts with the numbering of a whole elementary ring of the fullerene. Then, the numbering proceeds to cover all of the other atoms keeping the pitch of the helix as small as possible (keeping the movement "as tight as possible") and maintaining its clockwise or counterclockwise directionality.

\subsection{Numbering for $\left(C_{60}-I_{h}\right)[5,6]-$ and $\left(C_{70}-D_{5 h(6)}\right)[5,6]$ fullerene}

The following rules were adopted for numbering $\left(\mathrm{C}_{60}-I_{\mathrm{h}}\right)[5,6]$ fullerene and $\left(\mathrm{C}_{70}-D_{5 \mathrm{~h}(6)}\right)[5,6]$ fullerene [4].

Rule Fu-3.1.1: Proper rotation axes $\left(C_{\mathrm{n}}\right)$ are examined in sequence from the highest-order to the lowest-order axis, until at least one contiguous helical pathway is found that begins in a ring through which a proper rotation axis passes, or at one end of a bond bisected by a proper rotation axis, or at an atom through which a proper rotation axis passes. Numbering begins at the end of such a contiguous helical pathway, and the corresponding axis is called the "reference axis".

Rule Fu-3.1.2: If there is a choice for the selection of a reference axis or for the end of the reference axis to begin the numbering, a ring is preferred to a bond, which, in turn, is preferred to an atom.

Rule Fu-3.1.3: When there is a choice among helical numbering pathways, the preferred pathway terminates as close as possible, in terms of the number of bonds, to the reference axis.

The numberings of $\left(\mathrm{C}_{60}-I_{\mathrm{h}}\right)[5,6]-$ and $\left(\mathrm{C}_{70}-D_{5 \mathrm{~h}(6)}\right)[5,6]$ fullerene [4] illustrate the application of these rules.

\subsubsection{Systematic numbering for $\left(C_{60^{-I} h}\right)[5,6]$ fullerene (Fig. 1) (Atlas [5] Ref. No. 60:1; CAS Reg. No. 99685-96-8)}

This fullerene has six symmetry-equivalent $C_{5}$ axes passing through opposite pentagons, each of which gives identical contiguous helical pathways in either direction from any atom of any pentagon (all atoms are symmetry-equivalent in $\left(\mathrm{C}_{60}-I_{\mathrm{h}}\right)[5,6]$ fullerene $)$. Any of these $C_{5}$ axes can be the reference axis. According to rule $\mathbf{F u - 3 . 1 . 1}$, it is not necessary to consider any of the lower-order $C_{3}$ and $C_{2}$ axes. The systematic numbering is given in Fig. 1 . 


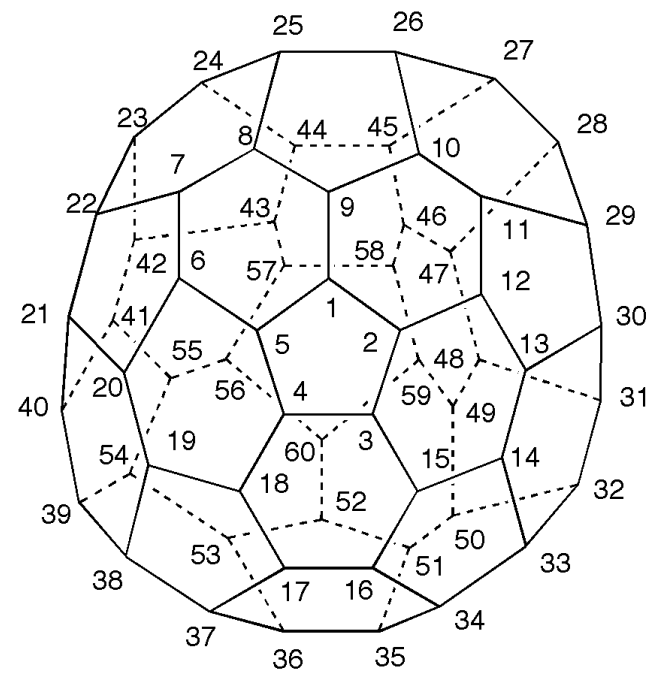

Fig. 1 Systematic numbering of $\left(\mathrm{C}_{60}-I_{\mathrm{h}}\right)[5,6]$ fullerene.

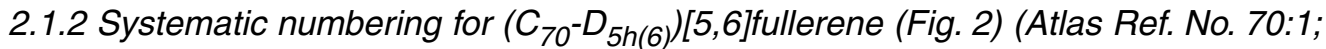

\section{CAS Reg. No. 115383-22-7)}

The principal axis for this fullerene is the $C_{5}$ axis passing through opposite pentagons. There are no contiguous helical pathways from any atom in either pentagon (all symmetry-equivalent), and, therefore, the $C_{5}$ axis cannot be the reference axis. Following rule $\mathbf{F u - 3 . 1 . 1}$, one of the five equivalent $C_{2}$ axes that pass through the center of a six-membered ring at one end and bisect the bond between two six-membered rings at the other end (see Fig. 2a) must be evaluated. Since, according to rule Fu-3.1.2, a ring is preferred to a bond for the beginning of numbering, the search for a helical pathway must start in the six-membered ring. A priori, there are 12 pathways, one in each direction, clockwise and counterclockwise, from each atom of the six-membered ring to be examined. However, because of symmetry,

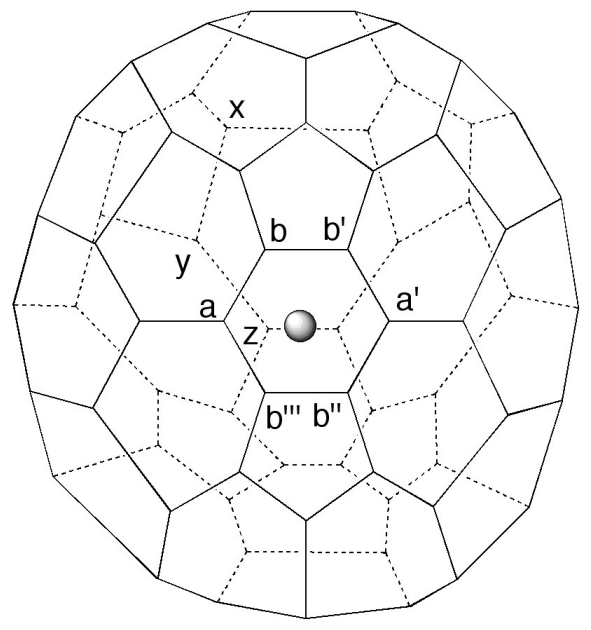

$2 \mathbf{a}$

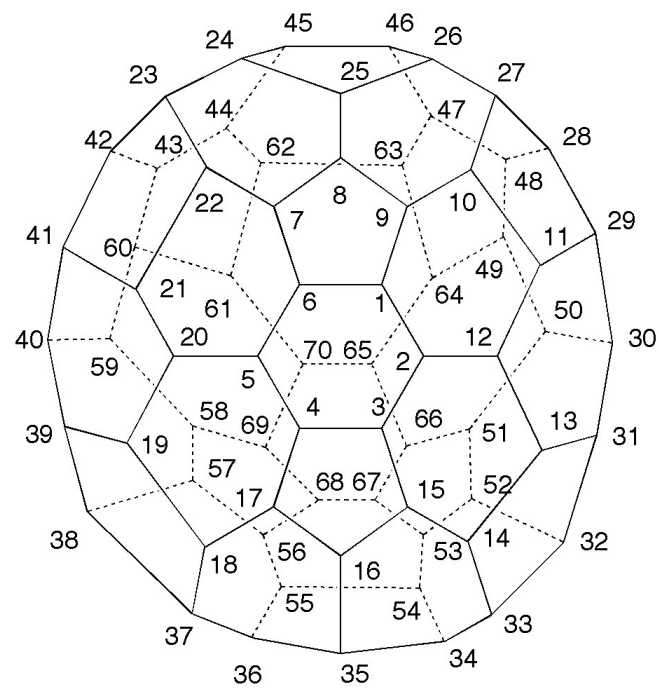

$\mathbf{2 b}$

Fig. 2 Systematic numbering of $\left(\mathrm{C}_{70}-D_{5 \mathrm{~h}(6)}\right)[5,6]$ fullerene. 
atoms $\mathbf{a}$ and $\mathbf{a}^{\prime}$ in Fig. 2a are equivalent, as are atoms $\mathbf{b}, \mathbf{b}^{\prime}, \mathbf{b}^{\prime \prime}$, and $\mathbf{b}^{\prime \prime \prime}$ (throughout this document, symmetry-related atoms are indicated by unprimed and primed identical letters; for the sake of clarity, however, atoms located at the end of a pathway are identified by letters such as $\mathbf{z} / \mathbf{y} / \mathbf{x} \ldots$ even if symmetryrelated to $\mathbf{a} / \mathbf{b} / \mathbf{c} \ldots .$.$) . Therefore, there are only three different pathways to explore: clockwise \mathbf{b}^{\prime}$ to $\mathbf{a}^{\prime}$ to $\mathbf{b}^{\prime \prime}$, clockwise $\mathbf{a}$ to $\mathbf{b}$ to $\mathbf{b}^{\prime}$, and clockwise $\mathbf{b}$ to $\mathbf{b}^{\prime}$ to $\mathbf{a}^{\prime}$. The pathway $\mathbf{b}^{\prime}$ to $\mathbf{a}^{\prime}$ to $\mathbf{b}^{\prime \prime}$ terminates at the atom marked $\mathbf{z}$ at the end of the bond bisected by the reference axis. The pathway $\mathbf{a}$ to $\mathbf{b}$ to $\mathbf{b}$ ' terminates at the atom marked $\mathbf{y}$, one bond removed from the bond at the end of the reference axis. The pathway $\mathbf{b}$ to $\mathbf{b}^{\prime}$ to $\mathbf{a}^{\prime}$ terminates at the atom marked $\mathbf{x}$, two bonds removed from the bond bisected by the reference axis. According to rule $\mathbf{F u - 3 . 1 . 3}$, the preferred contiguous pathway for numbering is $\mathbf{b}^{\prime}$ to $\mathbf{a}^{\prime}$ to $\mathbf{b}^{\prime \prime}$; the resulting systematic numbering is shown in Fig. $2 b$.

\section{NUMBERING OF FULLERENES OTHER THAN $\left(\mathrm{C}_{60} \mathrm{I}_{\mathrm{h}}\right)[5,6]-$ AND $\left(C_{70}-D_{5 h(6)}\right)[5,6]$ FULLERENE}

While rules Fu-3.1.1, Fu-3.1.2, and $\mathbf{F u - 3 . 1 . 3}$ are sufficient to systematically number $\left(\mathrm{C}_{60} \mathrm{I}_{\mathrm{h}}\right)[5,6]$ - and $\left(\mathrm{C}_{70}-D_{5 \mathrm{~h}(6)}\right)[5,6]$ fullerene, the numbering of other fullerenes may require additional rules. Some of these rules have already been proposed [4].

Rule Fu-3.1.2.1: When there is a choice between rings for the beginning of numbering, a larger ring is preferred to a smaller one. When there is still a choice, the preferred ring contains the highestranking atom at the first point of difference. The highest-ranking atom is defined as the common atom of the set of three largest rings. For example, in a [5,6]fullerene, an atom at the intersection of three sixmembered rings (in short, a 6,6,6 atom) ranks higher than an atom at the intersection of two six-membered rings and a five-membered ring (a 6,6,5 atom).

Rule Fu-3.1.2.2: When there is a choice between bonds bisected by an axis for the beginning of numbering, the preferred bond contains the higher number of higher-ranking atoms.

Rule Fu-3.1.2.3: When there is a choice between atoms lying on an axis for the beginning of numbering, the numbering must start at the higher-ranking atom.

In addition to the recommendations of ref. [4], one new rule was adopted:

Rule Fu-3.1.4: When there is still a choice among helical pathways for numbering, they are evaluated by sequential comparison of the ranking of their atoms. The preferred pathway contains the highest-ranking atom at the first point of difference.

Based on the rules required for the numbering of their atoms, fullerenes considered in this document are divided into the following classes:

3.1 Fullerenes having at least one symmetry axis $\left(C_{\mathrm{n}}, \mathrm{n}>1\right)$ and a contiguous helical numbering pathway determined on the basis of rules Fu-3.1.1 to Fu-3.1.4.

3.2 Fullerenes having at least one symmetry axis $\left(C_{\mathrm{n}}, \mathrm{n}>1\right)$, but no contiguous helical numbering pathway determined on the basis of rules $\mathbf{F u - 3 . 1 . 1}$ to $\mathbf{F u - 3 . 1 . 4}$.

3.3 Fullerenes belonging to the $C_{\mathrm{s}}$ point group and having a contiguous helical numbering pathway determined unambiguously as described in Section 3.3.

3.4 Fullerenes belonging to the $C_{\mathrm{i}}$ or $C_{1}$ point groups and having a contiguous helical numbering pathway determined unambiguously as described in Section 3.4.

\subsection{Fullerenes having at least one symmetry axis $\left(C_{n}, n>1\right)$ and a contiguous helical numbering pathway determined on the basis of rules Fu-3.1.1 to Fu-3.1.4}

There are fullerenes belonging to this class whose numbering can be devised solely on the basis of rules Fu-3.1.1 to Fu-3.1.3. Examples are given below. 


\subsubsection{Systematic numbering for $\left(C_{20^{-}}{ }_{h}\right)[5]$ fullerene (Fig. 3) (Atlas Ref. No. 20:1; CAS Reg. No. 104375-45-3)}

For this fullerene, any of the equivalent $C_{5}$ axes (the highest-order axis for this molecule), that pass through the centers of opposite faces, provide equivalent contiguous helical pathways in either direction from any atom of any pentagon (all atoms are symmetry-equivalent). Because this fullerene is achiral, the clockwise or counterclockwise direction of the helical numbering pattern is not important (caution: the direction of numbering may become important for chiral derivatives, see ref. [4]). Therefore, each of the $C_{5}$ axes can be the reference axis, and it is not necessary to examine the equivalent $C_{3}$ or equivalent $C_{2}$ axes, which are of lower order. The numbering for this fullerene is given in Fig. 3.

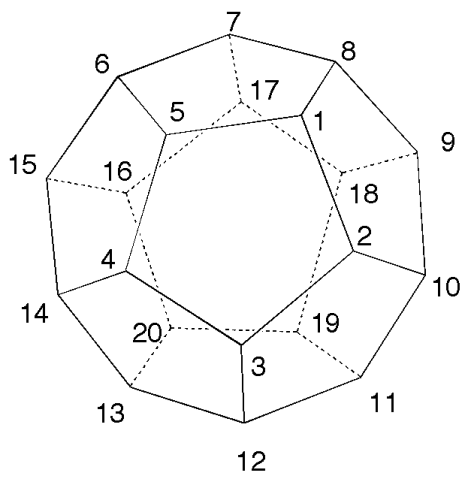

Fig. 3 Systematic numbering for $\left(\mathrm{C}_{20}-I_{\mathrm{h}}\right)[5]$ fullerene.

\subsubsection{Systematic numbering for $\left(C_{24}-O_{h}\right)[4,6] f u l l e r e n e$ (Fig. 4) (CAS Reg. No. 114153-97-8)}

This fullerene contains only 6,6,4 atoms. A contiguous helical pathway cannot be obtained using the three equivalent $C_{4}$ axes passing through opposite tetragonal faces (Fig. 4a). Therefore, following rule Fu-3.1.1, the four equivalent $C_{3}$ axes passing through opposite symmetry-equivalent hexagons are examined (Fig. 4b). Only the clockwise or counterclockwise pathways (both are equivalent for this achiral fullerene) starting along one of the three symmetry-equivalent bonds connecting symmetry-equivalent atoms (a and $\mathbf{a}$ ) lead to contiguous numberings. The clockwise numbering is shown in Fig. 4c. Pathways around the lower-order $C_{2}$ axes do not need to be considered.

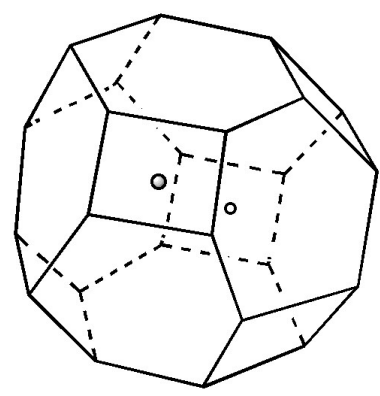

4a

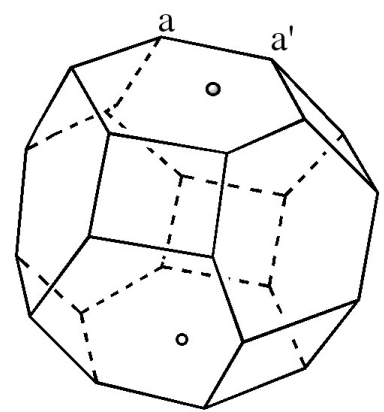

4b

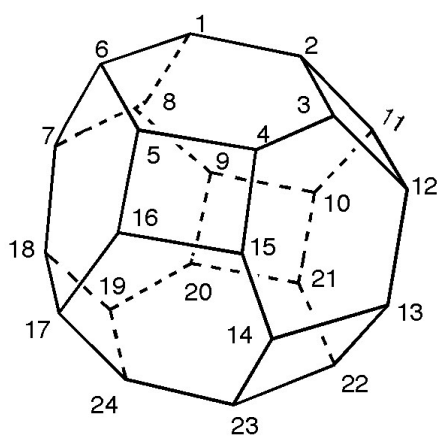

$4 c$

Fig. 4 Systematic numbering for $\left(\mathrm{C}_{24}-O_{\mathrm{h}}\right)[4,6]$ fullerene. 


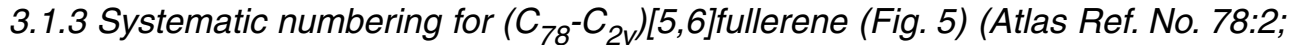 CAS Reg. No. 139707-96-3)}

This fullerene contains a single $C_{2}$ axis, which passes through the center of a six-membered ring on one side and the midpoint of a bond on the other side. According to rule Fu-3.1.2, the ring is preferred for beginning the numbering. Because of symmetry, there are only three pathways to be considered, namely, clockwise $\mathbf{a}$ to $\mathbf{b}$ to $\mathbf{a}^{\prime}, \mathbf{b}$ to $\mathbf{a}^{\prime}$ to $\mathbf{a}^{\prime \prime}$, and $\mathbf{a}^{\prime}$ to $\mathbf{a}^{\prime \prime}$ to $\mathbf{b}^{\prime}$ (Fig. 5a). These lead to the contiguous helical numberings shown in Figs. 5b, 5c, and 5d, respectively. By application of rule Fu-3.1.3, the numbering of Fig. $5 \mathrm{c}$ is preferred because it terminates at an atom belonging to the bond bisected by the $C_{2}$ axis, whereas the other numberings terminate at an atom one (Fig. 5d) and two (Fig. 5b) bonds removed from the $C_{2}$ axis, respectively.

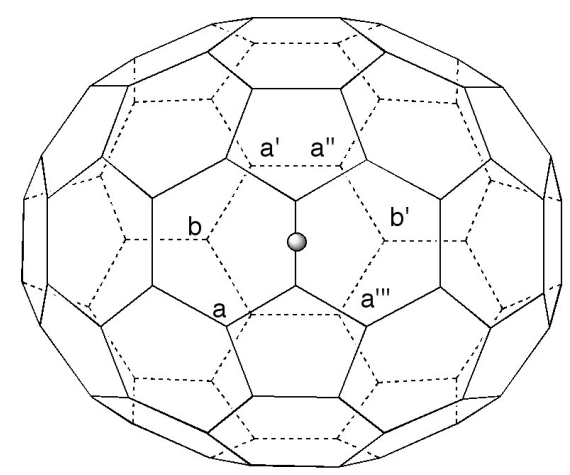

$5 \mathbf{a}$

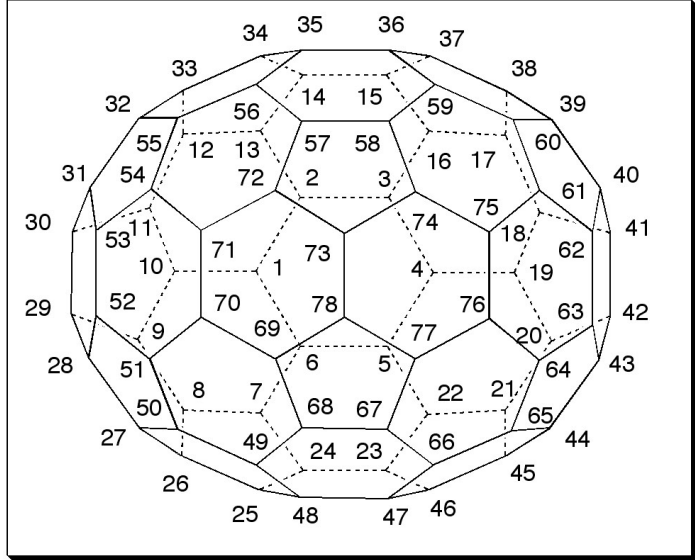

$5 \mathbf{c}$

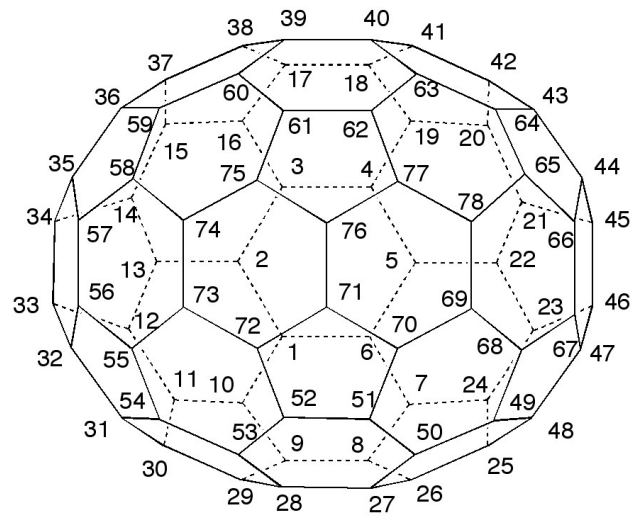

$\mathbf{5 b}$

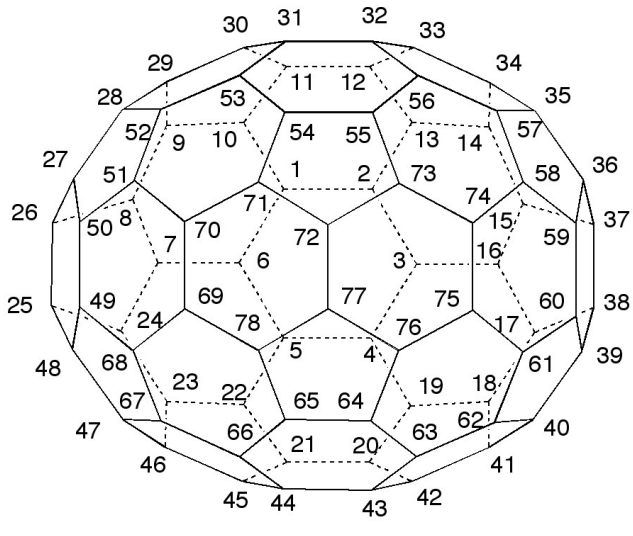

5d

Fig. 5 Systematic numbering for $\left(\mathrm{C}_{78}{ }^{-} C_{2 \mathrm{v}}\right)[5,6]$ fullerene.

\subsubsection{Systematic numbering for $\left(C_{48}-C_{2}\right)[5,6]$ fullerene (Fig. 6) (Atlas Ref. No. 48:163;}

CAS Reg. No. 150398-36-0; this fullerene is listed as $C_{1}$ by CAS)

This is a chiral fullerene. The discussion will consider the enantiomer shown in Fig. 6a. The only symmetry element in this fullerene is a $C_{2}$ axis, which bisects midpoints of opposite bonds (Fig. 6a). Both bonds connect 6,6,5 atoms ( $\mathbf{a}$ and $\mathbf{a}^{\prime}$ at the near end of the axis; $\mathbf{b}$ and $\mathbf{b}^{\prime}$ at the far end). Based on rule Fu-3.1.2.2, no selection between these bonds for beginning the numbering can be made. In Fig. 6a, the atoms indicated with the same letter (unprimed and primed) are identical for symmetry reasons. Also, 


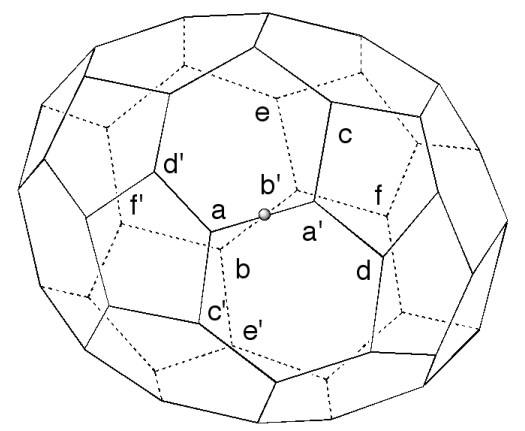

6a
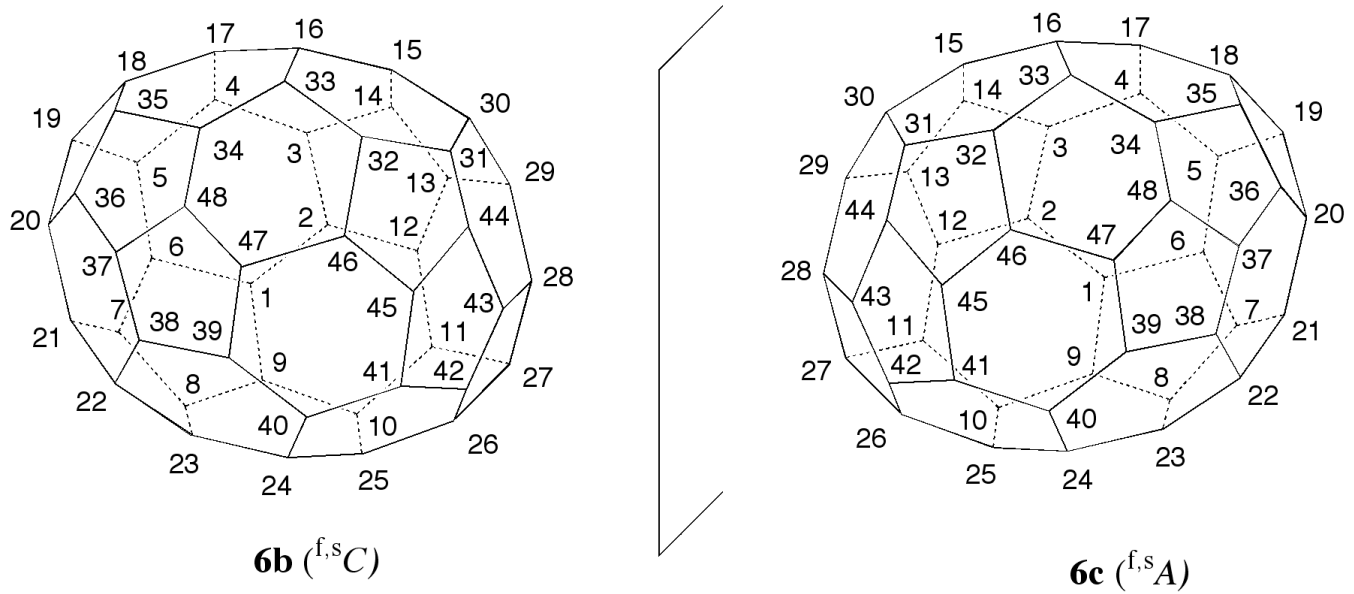

Fig. 6 Systematic numbering for the enantiomers of $\left(\mathrm{C}_{48}{ }^{-} C_{2}\right)[5,6]$ fullerene.

because of symmetry, only four pathways must be considered: $\mathbf{a}$ to $\mathbf{a}$ ' to $\mathbf{c} ; \mathbf{a}$ to $\mathbf{a}$ ' to $\mathbf{d} ; \mathbf{b}$ to $\mathbf{b}$ ' to $\mathbf{e}$; and $\mathbf{b}$ to $\mathbf{b}^{\prime}$ to $\mathbf{f}$. Of these, only the counterclockwise $\mathbf{b}$ to $\mathbf{b}^{\prime}$ to $\mathbf{e}$ pathway results in a contiguous helical numbering that is shown in Fig. $6 \mathrm{~b}$. Because the pathway is clockwise for a viewer looking directly at the polygon where the numbering begins from the outside of the fullerene cage, the stereodescriptor for this inherently chiral fullerene is $\left({ }^{\mathrm{f}, \mathrm{s}} C\right)$ (" $\mathrm{f} "=$ fullerene; "s" = systematic numbering; " $C$ " = clockwise) [4]. The numbering of the $\left({ }^{\mathrm{f}, \mathrm{s}} A\right)$ enantiomer, shown in Fig. $6 \mathrm{c}$, is the mirror-image of that shown in Fig. $6 \mathrm{~b}$.

\subsubsection{Systematic numbering for other fullerenes having at least one symmetry axis $\left(C_{n}, n>1\right)$ and a contiguous helical numbering pathway determined on the basis of rules \\ Fu-3.1.1 to Fu-3.1.4}

Fullerenes of this class do exist for which the application of rules Fu-3.1.1 to Fu-3.1.3 does not allow one to distinguish among several contiguous helical numbering pathways. To solve this problem, the additional rule Fu-3.1.4 has been adopted. The use of this new rule leads to numberings that in some cases are different from those obtained by CAS [3]. Examples of fullerenes whose numbering requires application of rule Fu-3.1.4 are described below. 


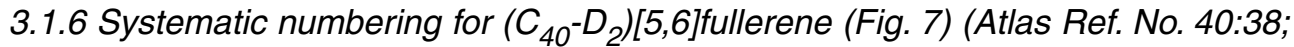 CAS Reg. No. 145646-78-2)}

This structure is characterized by three different axes. One axis passes through opposite six-membered rings; the other axes pass through midpoints of opposite bonds. By application of rule Fu-3.1.2, the axis passing through the ring has highest priority for selection as the reference axis. This axis is shown in Fig. 7a. The numbering should begin from one of the atoms belonging to either one of the two rings that are symmetry-equivalent. The six atoms of the ring form three pairs of symmetry-equivalent atoms: $\mathbf{a} / \mathbf{a}$ ', $\mathbf{b} / \mathbf{b}^{\prime}$, and $\mathbf{c} / \mathbf{c}^{\prime}$. Thus, there are only six pathways to be evaluated, clockwise and counterclockwise from $\mathbf{a}$, from $\mathbf{b}$, and from $\mathbf{c}$. The pathway $\mathbf{c}$ to $\mathbf{b}$ to $\mathbf{a}$ does not result in a contiguous helical numbering. All the other five pathways [a to $\mathbf{c}^{\prime}$ to $\mathbf{b}^{\prime}$ (Fig. 7b); $\mathbf{a}^{\prime}$ to $\mathbf{b}^{\prime}$ to $\mathbf{c}^{\prime}$ (Fig. 7c); $\mathbf{b}^{\prime}$ to $\mathbf{a}^{\prime}$ to $\mathbf{c}$ (Fig. 7d); $\mathbf{b}^{\prime}$ to $\mathbf{c}^{\prime}$ to $\mathbf{a}$ (Fig. 7e); $\mathbf{c}^{\prime}$ to $\mathbf{a}$ to $\mathbf{b}$ (Fig. 7f)] do result in contiguous numberings, and a choice must be made.

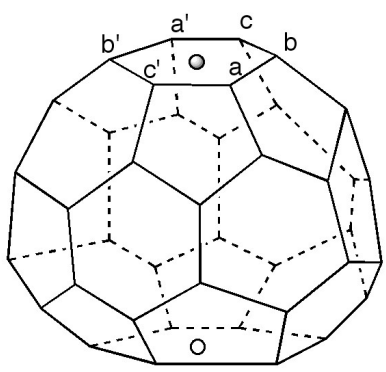

$7 \mathbf{a}$

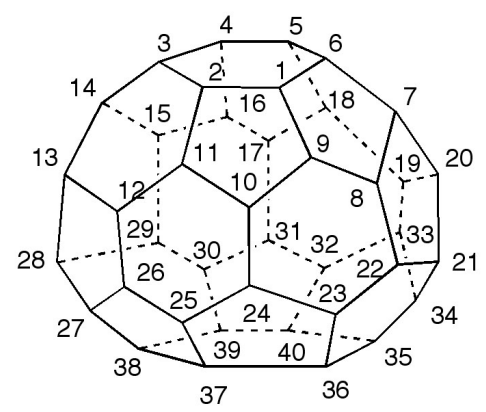

$7 \mathbf{b}$

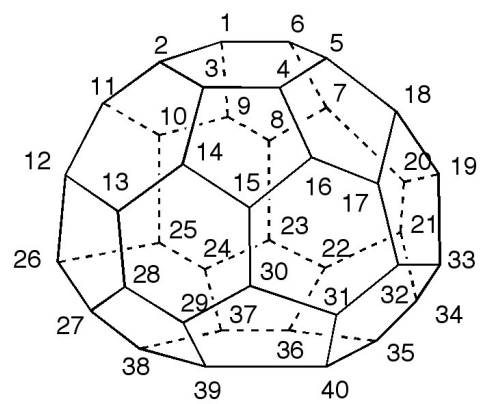

$7 \mathbf{c}$

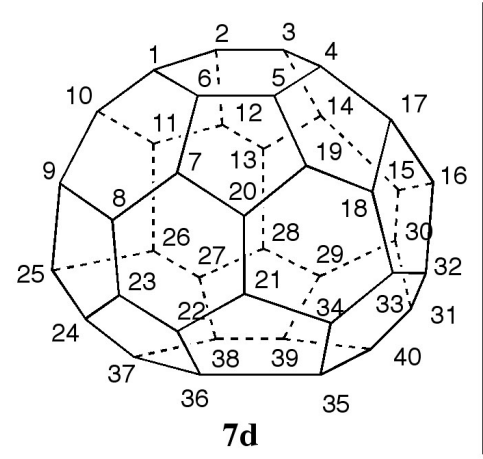

$7 d$

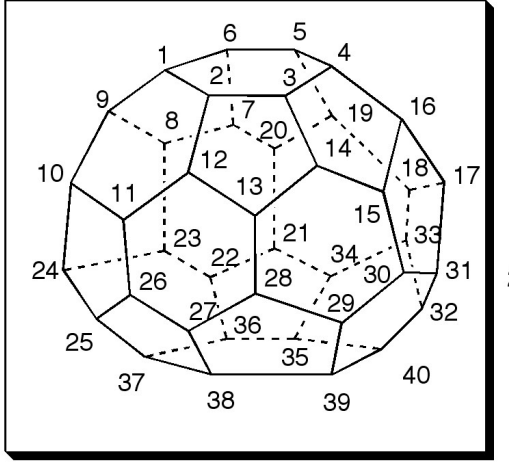

$7 \mathbf{e}$

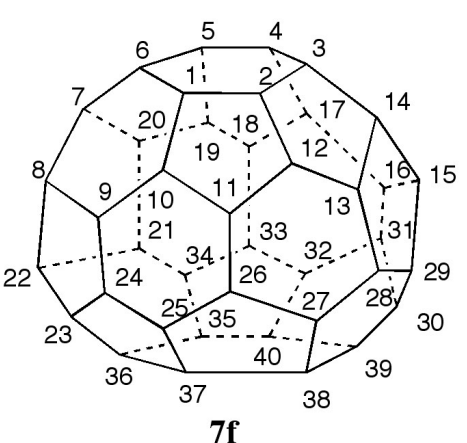

7f

Fig. 7 Systematic numbering for $\left({ }^{\mathrm{f}, \mathrm{s}} A\right)\left(\mathrm{C}_{40}-D_{2}\right)[5,6]$ fullerene.

Application of rules $\mathbf{F u}$-3.1.1 to $\mathbf{F u - 3 . 1 . 3}$ is not sufficient to select the preferred numbering, since all numberings terminate at an atom belonging to the ring intersected by the axis. Inspection of the atom-ranking using the new rule Fu-3.1.4 leads to selection of the pathway in Fig. 7e as the correct numbering since it contains the highest-ranking atom at the first point of difference (see Table 1). Indeed, the pathway in Fig. 7e begins at a 6,6,5 atom (and thus is preferred over the pathways in Figs. $7 \mathrm{~b}$ and $7 \mathrm{c}$ ), and contains a 6,6,5 atom at position 2, whereas both pathways in Figs. $7 \mathrm{~d}$ and $7 \mathrm{f}$ contain a $6,5,5$ atom at the same position. The stereodescriptor for the shown enantiomer of this inherently chiral fullerene is $\left({ }^{\mathrm{f}, \mathrm{s}} \mathrm{A}\right)[4]$. Note that, once a numbering is discarded, further inspection of atom-ranking 
is not required. Based on different rules [3], CAS selects the pathway in Fig. 7c as the preferred numbering.

Table 1 Rankings of atoms 1 and 2 for pathways in Figs. 7b-7f.

\begin{tabular}{lccccc}
\hline Atom & $7 \mathrm{~b}$ & $7 \mathrm{c}$ & $7 \mathrm{~d}$ & $7 \mathrm{e}$ & $7 \mathrm{f}$ \\
\hline 1 & $6,5,5$ & $6,5,5$ & $\mathbf{6 , 6 , 5}$ & $\mathbf{6 , 6 , 5}$ & $\mathbf{6 , 6 , 5}$ \\
2 & - & - & $6,5,5$ & $\mathbf{6 , 6 , 5}$ & $6,5,5$ \\
\hline
\end{tabular}

3.1.7 Systematic numbering for $\left(C_{76}{ }^{-D_{2}}\right)[5,6]$ fullerene (Fig. 8) (Atlas Ref. No. 76:1; CAS Reg. No. 142136-39-8)

This chiral fullerene has three axes, each intersecting the midpoint of a bond. These axes are indicated in Figs. 8a, 8b, and 8c. Application of rule Fu-3.1.2.2 allows one to discard the axis in Fig. 8b because it bisects bonds connecting only 6,6,5 atoms, whereas in the other cases, the corresponding bonds connect 6,6,6 atoms. The two bonds at the end of each reference axis in Figs. 8a and 8c are symmetryequivalent, and only the inspection of the pathways originating from the atoms of one of these bonds is necessary. For symmetry reasons, there are two possible beginnings for the numbering in each Fig. 8a and Fig. 8c. These have been indicated as $\mathbf{a}$ to $\mathbf{a}^{\prime}$ to $\mathbf{b}$, and $\mathbf{a}$ to $\mathbf{a}^{\prime}$ to $\mathbf{c}$ in each of these figures. Thus, there are four pathways to be considered. All of them are contiguous. The $\mathbf{a}$ to $\mathbf{a}$ to $\mathbf{b}$ numbering in Fig. $8 \mathrm{c}$ terminates at an atom one bond removed from the bond bisected by the reference axis; the a to $\mathbf{a}^{\prime}$ to c numbering in Fig. 8c terminates at an atom two bonds removed from the bond bisected by the axis; the two numberings in Fig. 8a terminate at atoms belonging to the bond bisected by the axis. According to rule Fu-3.1.3, the latter two numberings, shown in Figs. 8d and 8e, are preferred pathways, but a selection must still be made between them. Inspection of the ranking of the atoms according to rule Fu-3.1.4 (Table 2) shows that the atom at position 3 of Fig. 8 d is a 6,6,5 atom, whereas the atom at the same position in Fig. 8e is a 6,6,6 atom. Therefore, the numbering of Fig. 8e is preferred for the shown structure. In this case, the numbering is the same as that selected by CAS [3]. The stereodescriptor for the shown enantiomer of this inherently chiral fullerene is $\left({ }^{\mathrm{f}, \mathrm{s}} C\right)[4]$.

Table 2 Rankings of atoms 1 to 3 for pathways in Figs. 8d and 8e.

\begin{tabular}{lcc}
\hline Atom & $8 \mathrm{~d}$ & $8 \mathrm{e}$ \\
\hline 1 & $6,6,6$ & $6,6,6$ \\
2 & $6,6,6$ & $6,6,6$ \\
3 & $6,6,5$ & $\mathbf{6 , 6 , 6}$ \\
\hline
\end{tabular}




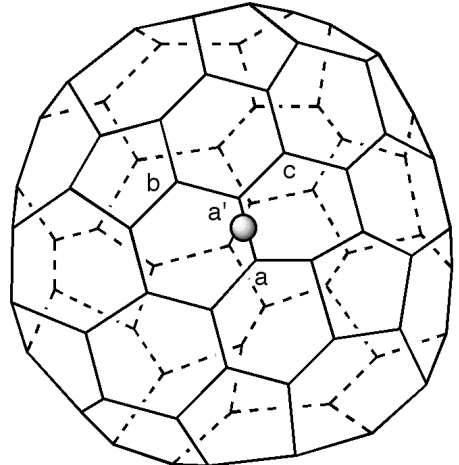

8a

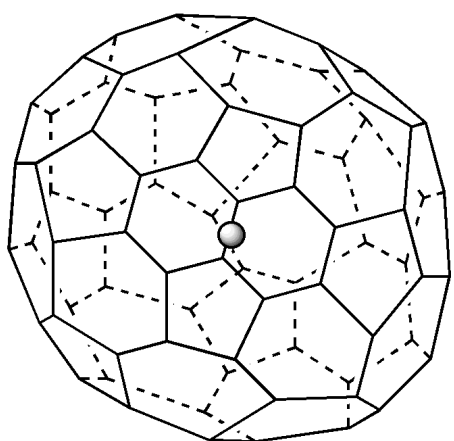

$\mathbf{8 b}$

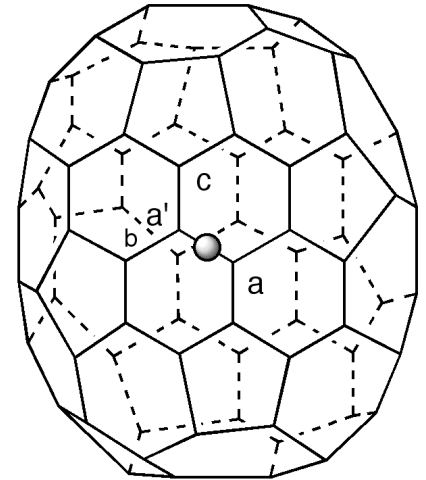

8c

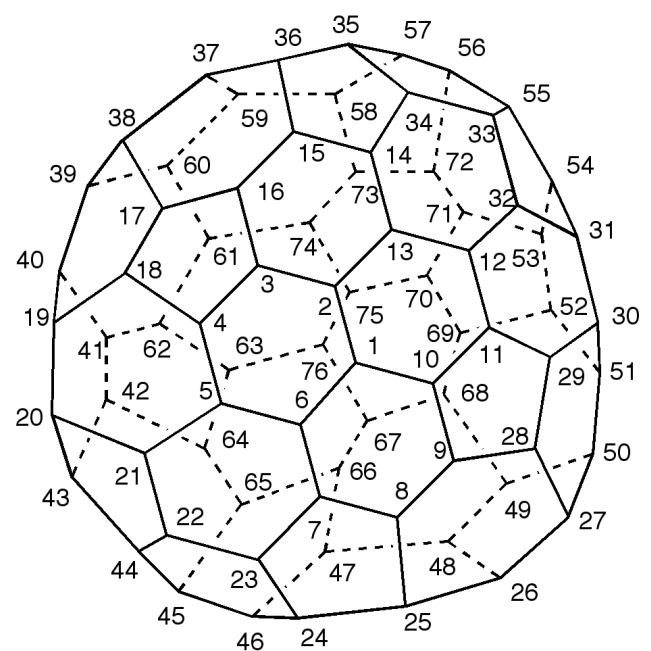

8d

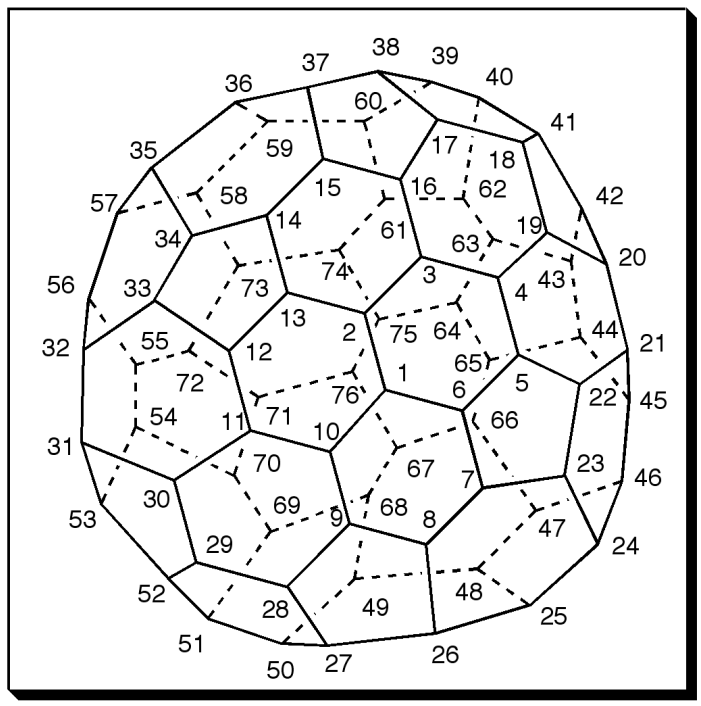

$\mathbf{8 e}$

Fig. 8 Systematic numbering for $\left({ }^{\mathrm{f}, \mathrm{s}} C\right)\left(\mathrm{C}_{76}-D_{2}\right)[5,6]$ fullerene.

\subsubsection{Systematic numbering for $\left(C_{28}-D_{2}\right)[5,6]$ fullerene (Fig. 9) (Atlas Ref. No. 28:1; CAS Reg. No. 145393-14-2)}

This chiral fullerene has three $C_{2}$ axes, shown in Figs. 9a, 9b, and 9c, respectively. The axis of Fig. 9a is preferred because it passes through the midpoint of two bonds connecting 6,6,5 atoms, whereas both the other axes pass through the midpoints of two bonds connecting lower-ranking atoms (rule $\mathbf{F u}-3.1 .2 .2)$. Because of symmetry, only two possible pathways must be considered, namely $\mathbf{a}$ to $\mathbf{a}$ ' to $\mathbf{b}$ and $\mathbf{a}$ to $\mathbf{a}$ to $\mathbf{c}$ (Fig. 9a). The resulting helical numberings are shown in Figs. 9d and 9e, respectively. Both numberings terminate at the same atom and application of rule Fu-3.1.3 is inconclusive. Application of rule Fu-3.1.4 (Table 3) shows that the two numberings differ at position 15, where there is a 6,5,5 atom in the numbering of Fig. 9d, but a 5,5,5 atom in the numbering of Fig. 9e. Therefore, the numbering of Fig. 9d is preferred. CAS selects the numbering of Fig. 9e [3]. The stereodescriptor for the shown enantiomer of this inherently chiral fullerene is $\left({ }^{\mathrm{f}, \mathrm{s}} C\right)[4]$. 


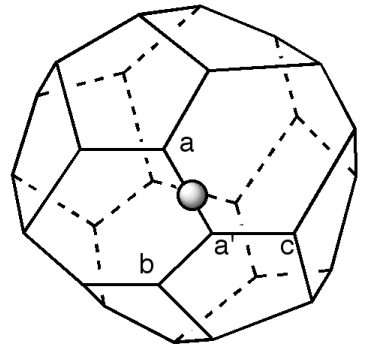

9a

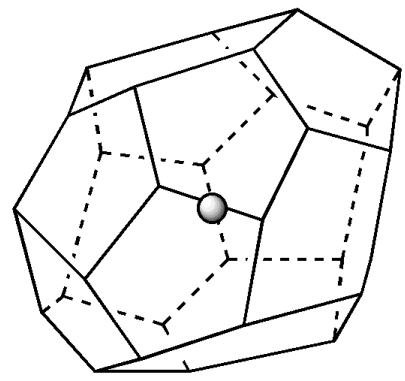

9b

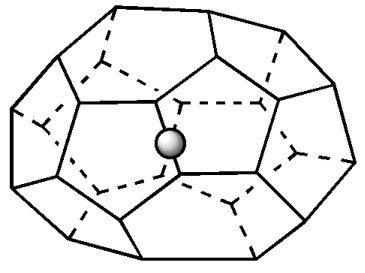

$9 c$

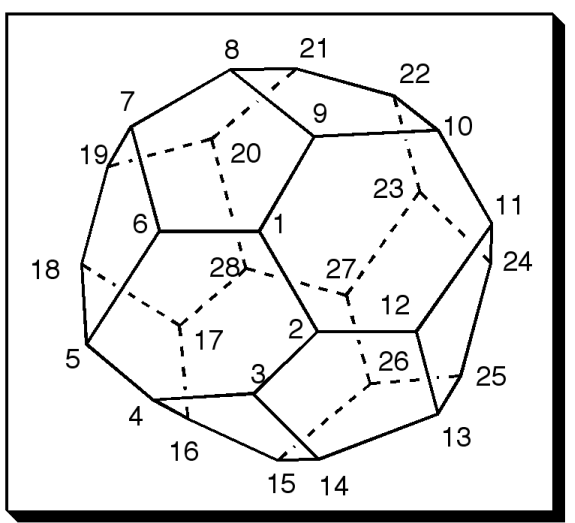

9d

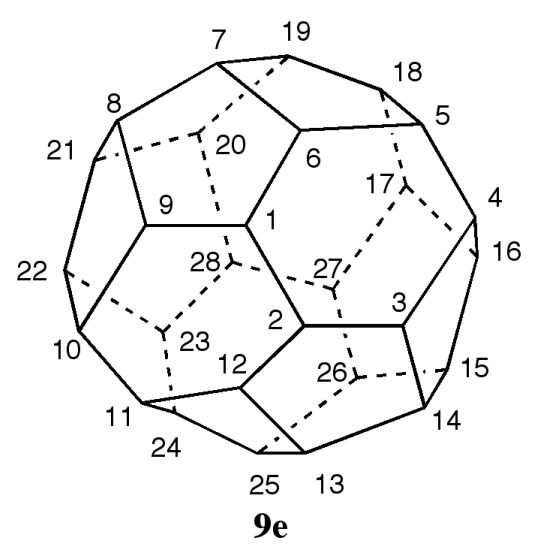

9 e

Fig. 9 Systematic numbering for $\left.{ }^{\mathrm{f}, \mathrm{s}} C\right)\left(\mathrm{C}_{28}-D_{2}\right)[5,6]$ fullerene.

Table 3 Rankings of atoms 1 to 15 for pathways in Figs. 9d and 9e.

\begin{tabular}{ccc}
\hline Atom & $9 \mathrm{~d}$ & $9 \mathrm{e}$ \\
\hline 1 & $6,6,5$ & $6,6,5$ \\
2 & $6,6,5$ & $6,6,5$ \\
3 & $6,5,5$ & $6,5,5$ \\
4 & $6,5,5$ & $6,5,5$ \\
5 & $6,5,5$ & $6,5,5$ \\
6 & $6,5,5$ & $6,5,5$ \\
7 & $5,5,5$ & $5,5,5$ \\
8 & $5,5,5$ & $5,5,5$ \\
9 & $6,5,5$ & $6,5,5$ \\
10 & $6,5,5$ & $6,5,5$ \\
11 & $6,5,5$ & $6,5,5$ \\
12 & $6,5,5$ & $6,5,5$ \\
13 & $5,5,5$ & $5,5,5$ \\
14 & $5,5,5$ & $5,5,5$ \\
15 & $\mathbf{6 , 5 , 5}$ & $5,5,5$ \\
\hline
\end{tabular}




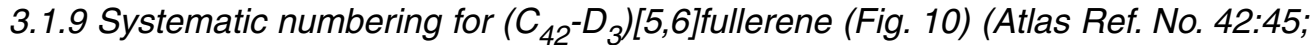
CAS Reg. No. 145646-79-3)

The highest-order axis of this chiral fullerene is the axis passing through opposite and symmetry-equivalent six-membered rings (Fig. 10a). Numbering can begin in either one of these rings, the six atoms of which are symmetry-equivalent in two sets of three: $\mathbf{a}$ is equivalent to $\mathbf{a}^{\prime}$ and $\mathbf{a} "$, and $\mathbf{b}$ is equivalent to $\mathbf{b}^{\prime}$ and $\mathbf{b}^{\prime \prime}$. As a consequence, there are four possible pathways to be considered: $\mathbf{a}$ to $\mathbf{b}$ to $\mathbf{a}^{\prime} ; \mathbf{a}$ to $\mathbf{b}^{\prime \prime}$ to $\mathbf{a}^{\prime \prime} ; \mathbf{b}$ to $\mathbf{a}^{\prime}$ to $\mathbf{b}^{\prime} ; \mathbf{b}$ to $\mathbf{a}$ to $\mathbf{b}^{\prime \prime}$. The pathway $\mathbf{b}$ to $\mathbf{a}^{\prime}$ to $\mathbf{b}^{\prime}$ does not lead to a contiguous numbering, whereas the other three pathways do. These are reported in Figs. 10b (a to $\mathbf{b}$ to $\mathbf{a}^{\prime}$ ), 10c (a to $\mathbf{b}^{\prime \prime}$ to $\mathbf{a} "$ ), and $10 \mathrm{~d}$ (b to $\mathbf{a}$ to $\mathbf{b}^{\prime \prime}$. Since all three pathways terminate at an atom belonging to the six-membered ring bisected by the reference axis, application of rule Fu-3.1.3 is inconclusive. Inspection of the atom se-

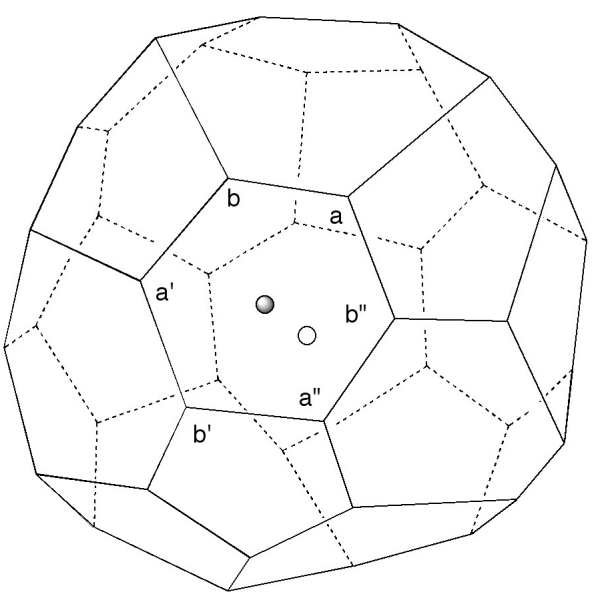

$10 \mathbf{a}$

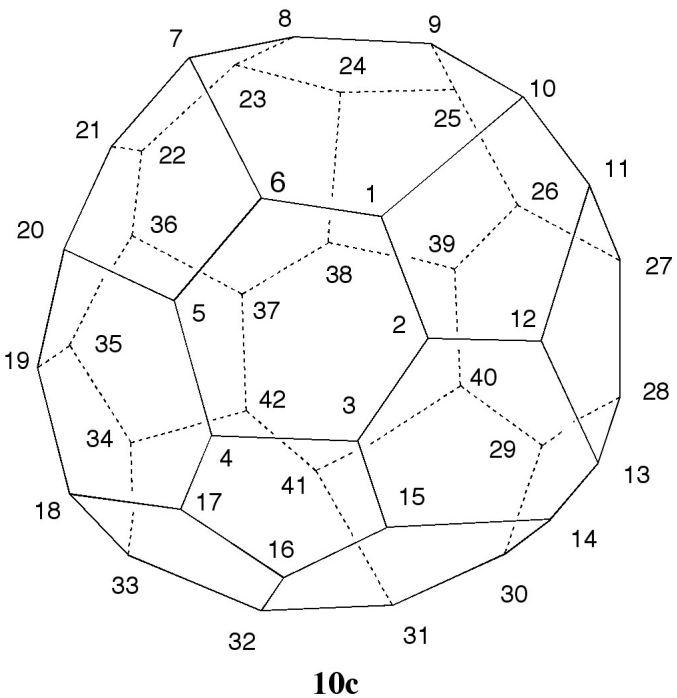

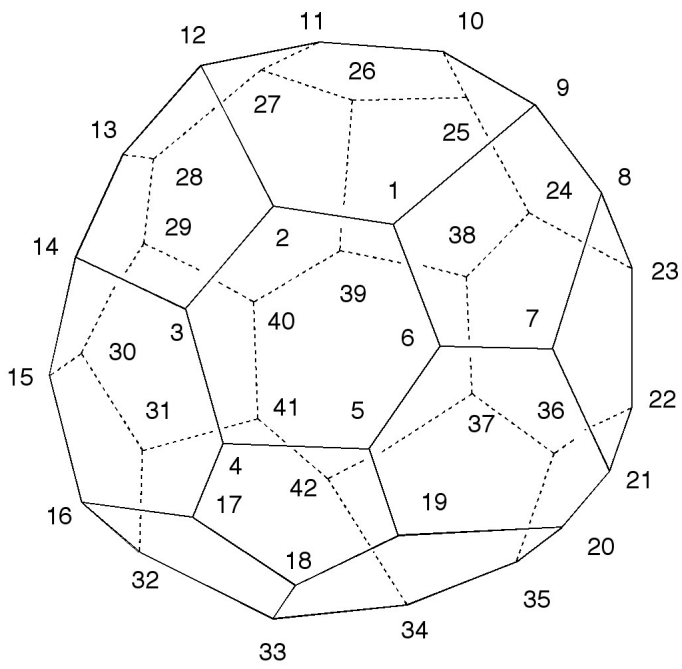

$10 b$

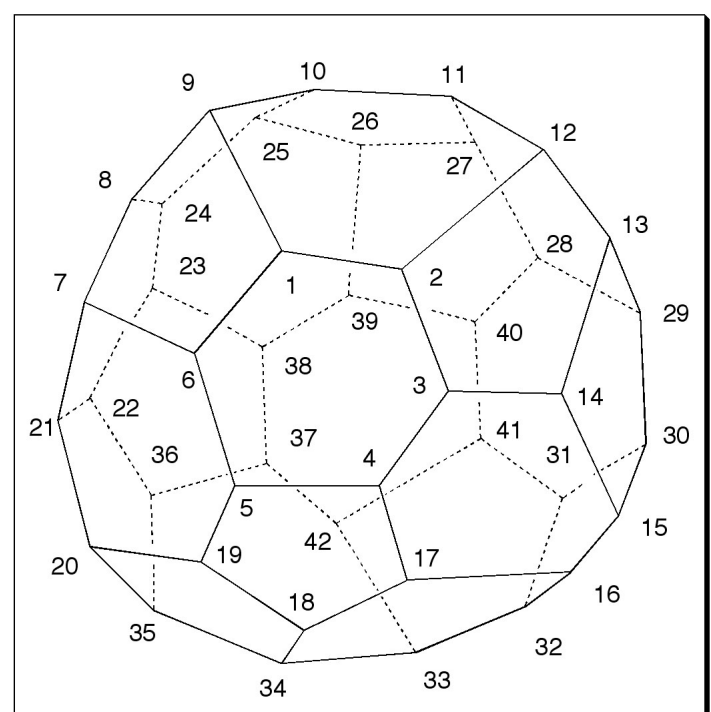

10d

Fig. 10 Systematic numbering for $\left({ }^{\mathrm{f}, \mathrm{s}} C\right)\left(\mathrm{C}_{42}-D_{3}\right)[5,6]$ fullerene. 
quences of Figs. 10b, 10c, and 10d according to rule Fu-3.1.4 (Table 4) shows that the sequence in Fig. 10d has a 6,6,5 atom at position 7, whereas those in Figs. 10b and 10c both have a 6,5,5 atom at the same position. The numbering of Fig. 10d is, therefore, preferred. CAS selects the same numbering [3]. The stereodescriptor for the shown enantiomer of this inherently chiral fullerene is $\left({ }^{\mathrm{f}, \mathrm{s}} C\right)$ [4].

Table 4 Rankings of atoms 1 to 7 for pathways in Figs. 10b, 10c, and 10d.

\begin{tabular}{lccc}
\hline Atom & $10 \mathrm{~b}$ & $10 \mathrm{c}$ & $10 \mathrm{~d}$ \\
\hline 1 & $6,6,5$ & $6,6,5$ & $6,6,5$ \\
2 & $6,6,5$ & $6,6,5$ & $6,6,5$ \\
3 & $6,6,5$ & $6,6,5$ & $6,6,5$ \\
4 & $6,6,5$ & $6,6,5$ & $6,6,5$ \\
5 & $6,6,5$ & $6,6,5$ & $6,6,5$ \\
6 & $6,6,5$ & $6,6,5$ & $6,6,5$ \\
7 & $6,5,5$ & $6,5,5$ & $\mathbf{6 , 6 , 5}$ \\
\hline
\end{tabular}

\subsubsection{Systematic numbering for $\left(C_{34}-C_{2}\right)[5,6]$ fullerene (Fig. 11) (Atlas Ref. No. 34:5; CAS Reg. No. 145646-75-9)}

The only $C_{2}$ axis in this fullerene is the reference axis. It passes through the center of the six-membered ring at the far end and bisects a bond at the near end of the structure in Fig. 11a. According to rule Fu-3.1.2, the numbering should begin in the ring which contains three pairs of symmetry-equivalent atoms indicated as $\mathbf{a} / \mathbf{a},, \mathbf{b} / \mathbf{b}^{\prime}$, and $\mathbf{c} / \mathbf{c}^{\prime}$. There are six possible pathways to be evaluated: $\mathbf{a}$ to $\mathbf{b}$ to $\mathbf{c} ; \mathbf{a}$ to $\mathbf{c}^{\prime}$ to $\mathbf{b}^{\prime} ; \mathbf{b}$ to $\mathbf{c}$ to $\mathbf{a}^{\prime} ; \mathbf{b}$ to $\mathbf{a}$ to $\mathbf{c}^{\prime} ; \mathbf{c}$ to $\mathbf{a}^{\prime}$ to $\mathbf{b}^{\prime} ;$ and $\mathbf{c}$ to $\mathbf{b}$ to $\mathbf{a}$. Of these, $\mathbf{a}$ to $\mathbf{b}$ to $\mathbf{c}$ and $\mathbf{b}$ to $\mathbf{a}$ to $\mathbf{c}^{\prime}$ do not yield a contiguous helical numbering pathway, whereas the other four pathways do. Since they terminate at an atom belonging to the bond bisected by the $C_{2}$ axis, a selection among them cannot be made using rule $\mathbf{F u - 3 . 1 . 3 . ~ H o w e v e r , ~ a p p l i c a t i o n ~ o f ~ r u l e ~} \mathbf{F u - 3 . 1 . 4}$ solves the problem. First, pathways $\mathbf{b}$ to $\mathbf{c}$ to $\mathbf{a}^{\prime}$ (Fig. 11b) and $\mathbf{a}$ to $\mathbf{c}^{\prime}$ to $\mathbf{b}^{\prime}$ (Fig. 11c), that begin with 6,6,5 atoms, are preferred over the other two pathways that begin with 6,5,5 atoms. Then, inspection of the atom-ranking shows that the numbering in Fig. $11 \mathrm{~b}$ is preferred to that in Fig. 11c since it contains a 6,6,5 rather than a 6,5,5 atom at position 21. The stereodescriptor for the shown enantiomer of this inherently chiral fullerene is $\left({ }^{\mathrm{f}, \mathrm{s}} A\right)$ [4].

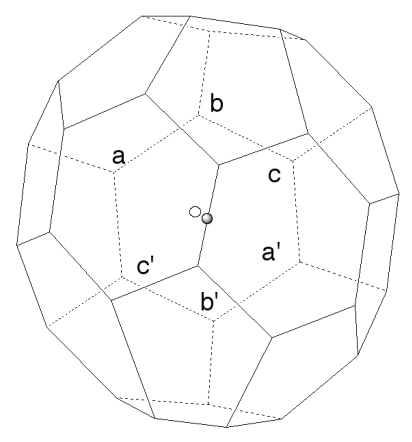

$11 \mathbf{a}$

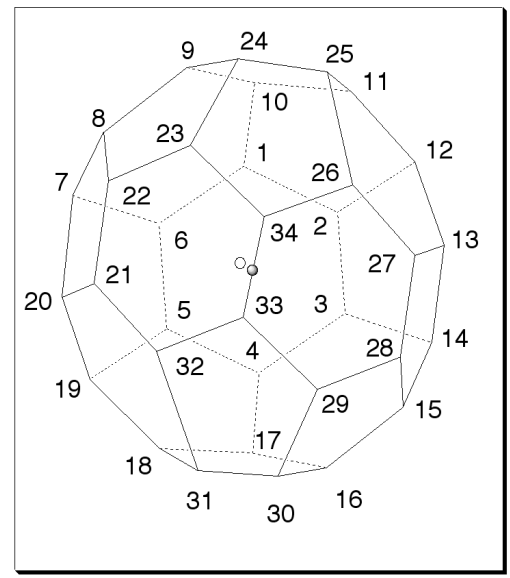

$11 \mathrm{~b}$

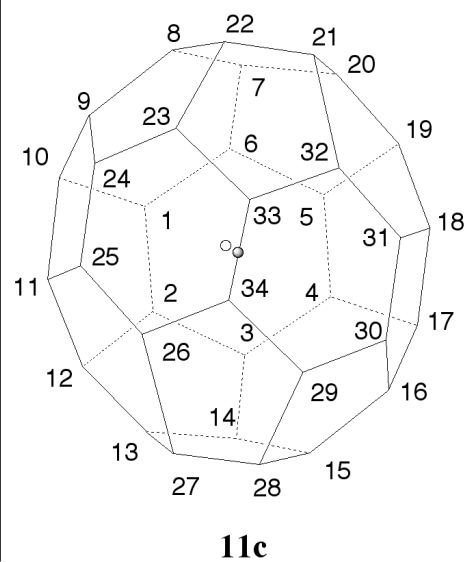

$11 \mathrm{c}$

Fig. 11 Systematic numbering for $\left({ }^{\mathrm{f}, \mathrm{s}} A\right)\left(\mathrm{C}_{34}-C_{2}\right)[5,6]$ fullerene. 
3.1.11 Systematic numbering for $\left(C_{78}-D_{3}\right)[5,6]$ fullerene (Fig. 12) (Atlas Ref. No. 78:1; CAS Reg. No. 139707-95-2)

This fullerene contains one $C_{3}$ axis, passing through the center of opposite and symmetry-equivalent six-membered rings (Fig. 12a), and three $C_{2}$ axes, perpendicular to the $C_{3}$ axis. According to rule Fu-3.1.1, the highest-order axis $\left(C_{3}\right)$ must be examined first for contiguous helical pathways. The atoms of the six-membered ring are symmetry-equivalent in sets of three $\left(\mathbf{a} / \mathbf{a}^{\prime} / \mathbf{a}^{\prime \prime}\right.$ and $\left.\mathbf{b} / \mathbf{b}^{\prime} / \mathbf{b}^{\prime \prime}\right)$. There are four

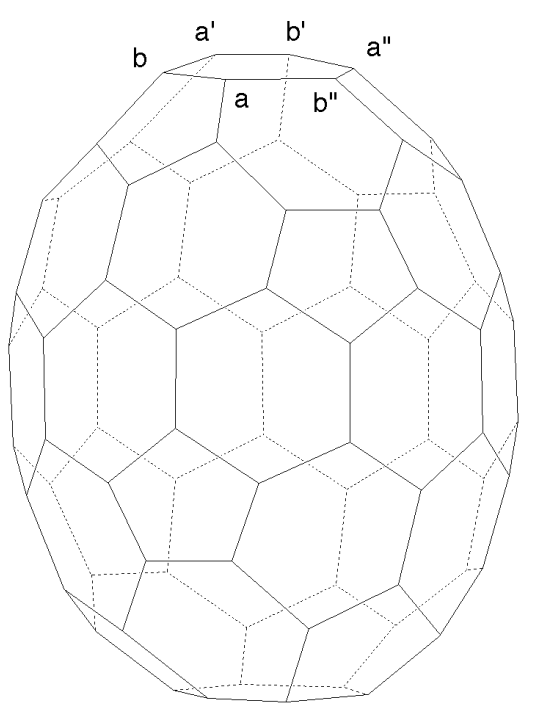

12a

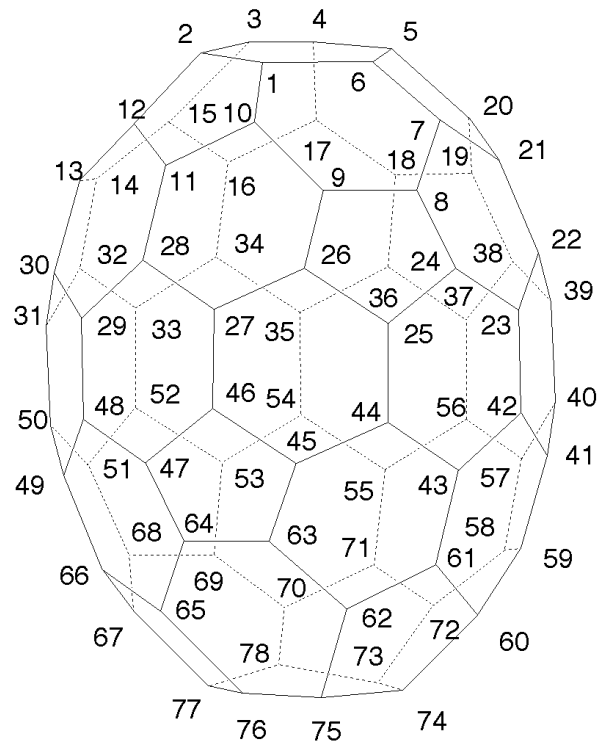

$12 \mathrm{c}$

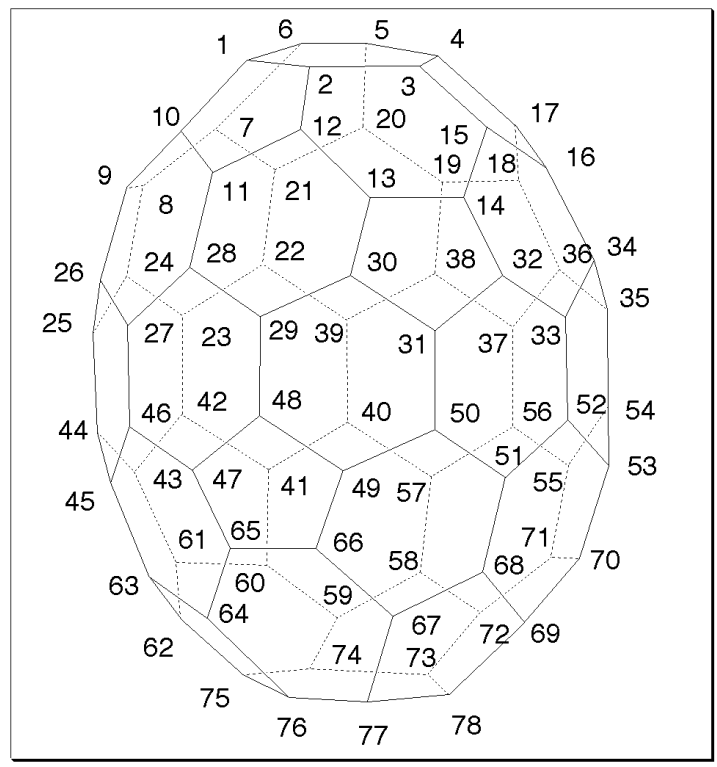

$12 \mathrm{~b}$

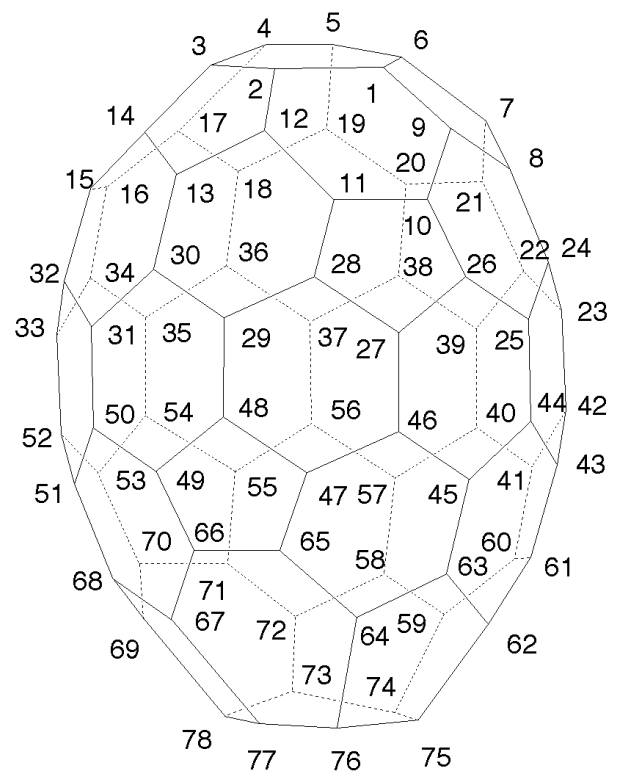

12d

Fig. 12 Systematic numbering for $\left({ }^{\mathrm{f}, \mathrm{s}} A\right)\left(\mathrm{C}_{78^{-}} D_{3}\right)[5,6]$ fullerene. 
possible pathways to be considered: $\mathbf{b}$ to $\mathbf{a}$ to $\mathbf{b}^{\prime \prime} ; \mathbf{a}$ to $\mathbf{b}$ to $\mathbf{a}^{\prime} ; \mathbf{b}^{\prime \prime}$ to $\mathbf{a}$ to $\mathbf{b}$; and $\mathbf{a}$ to $\mathbf{b}^{\prime \prime}$ to $\mathbf{a}$ ". Only the first three (shown in Figs. 12b, 12c, and 12d) lead to contiguous helical numberings terminating at an atom belonging to the six-membered ring intersected by the $C_{3}$ axis. Rule Fu-3.1.3 does not select among these numberings. Application of rule Fu-3.1.4 first eliminates the numbering of Fig. 12d, in which there is a 6,6,5 atom at position 22 rather than a 6,6,6 atom as in the numberings of Figs. 12b and 12c. By continuing the atom-ranking exploration for $12 \mathrm{~b}$ and $12 \mathrm{c}$, the numbering in Fig. 12b is preferred over that in Fig. 12c because it features a 6,6,6 rather than a 6,6,5 atom at position 40. The stereodescriptor for the shown enantiomer of this inherently chiral fullerene is $\left({ }^{\mathrm{f}, \mathrm{s}} A\right)$ [4].

\subsubsection{Systematic numbering for $\left(C_{78}-C_{2 v}\right)[5,6] f u l l e r e n e$ (Fig. 13) (Atlas Ref. No. 78:3;}

\section{CAS Reg. No. 143974-29-2)}

This fullerene contains a single $C_{2}$ axis at the intersection of two mirror planes; the axis passes through the center of a six-membered ring on one side and the midpoint of a bond on the other side. According to rule $\mathbf{F u - 3 . 1 . 2 , ~ t h e ~ r i n g ~ i s ~ p r e f e r r e d ~ f o r ~ b e g i n n i n g ~ t h e ~ n u m b e r i n g . ~ B e c a u s e ~ o f ~ s y m m e t r y , ~ t h e r e ~ a r e ~ o n l y ~}$ three pathways to be considered, namely, $\mathbf{a}$ to $\mathbf{b}$ to $\mathbf{c}, \mathbf{b}$ to $\mathbf{c}$ to $\mathbf{a}^{\prime}$, and $\mathbf{c}$ to $\mathbf{a}^{\prime}$ to $\mathbf{b}^{\prime}$ (Fig. 13a). The first
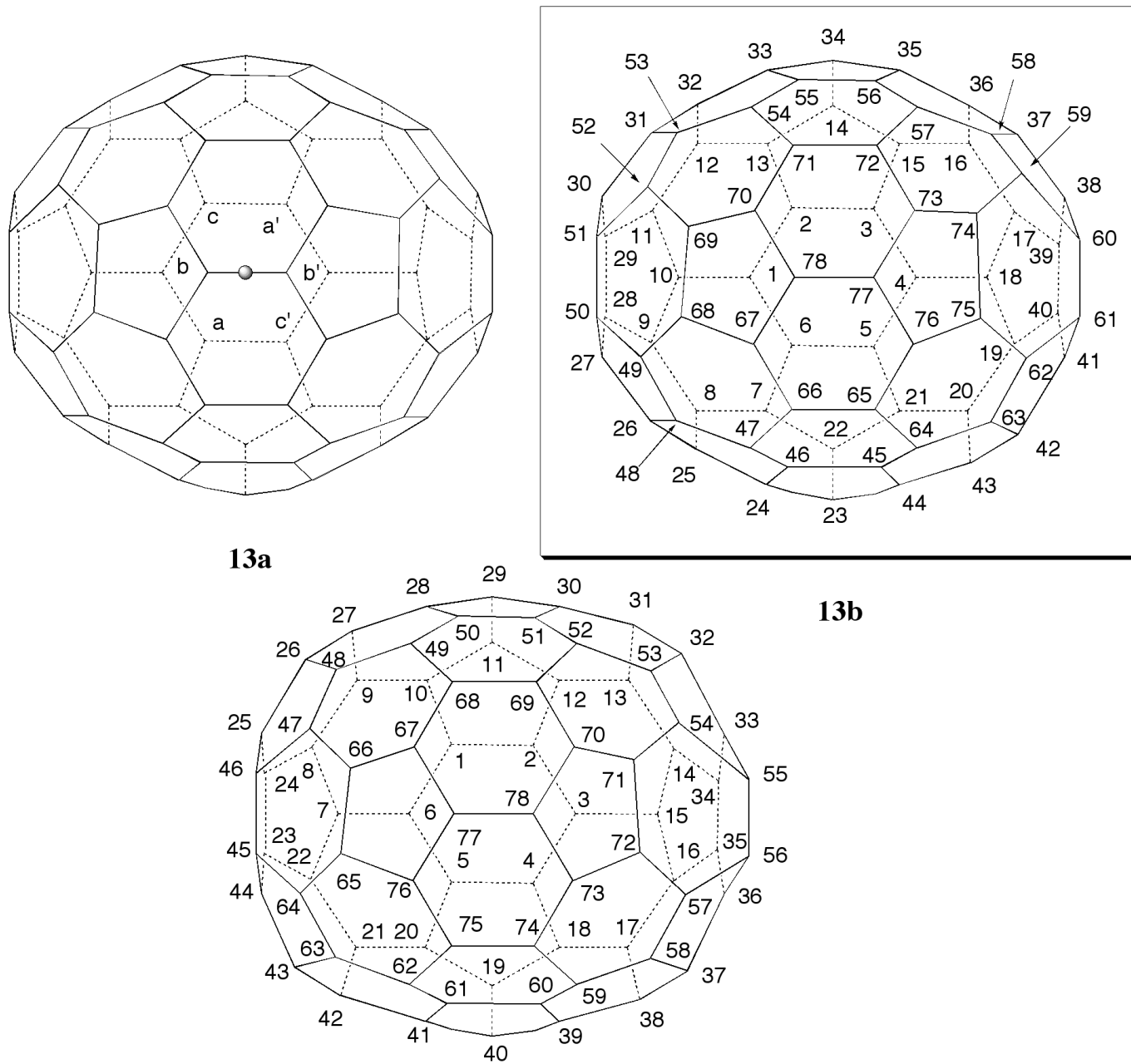

\section{$13 \mathbf{c}$}

Fig. 13 Systematic numbering for $\left(\mathrm{C}_{78^{-}} C_{2 \mathrm{v}}\right)[5,6]$ fullerene. 
pathway does not lead to a contiguous helical numbering. The other two (Figs. 13b and 13c) do. Both terminate at an atom belonging to the bond bisected by the reference axis, and application of rule Fu-3.1.3 is inconclusive. By inspection of the atom-ranking according to rule $\mathbf{F u - 3 . 1 . 4}$, the numbering of Fig. 13b is preferred because it begins with a 6,6,6 rather than with a 6,6,5 atom (Fig. 13c).

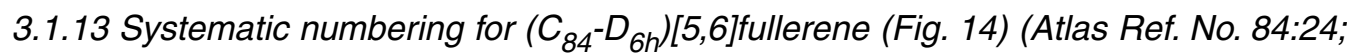
CAS Reg. No. 134847-09-9)

In this fullerene, the principal axis is the $C_{6}$ axis passing through the midpoint of opposite and symmetry-equivalent hexagons (Fig. 14a). However, no contiguous helical numbering can be found starting from the six symmetry-equivalent atoms of one of these rings. Therefore, the six $C_{2}$ axes must be considered. Three of these are symmetry-equivalent axes passing through the midpoint of opposite hexagons (one is shown by black and white dots in Fig. 14a); the remaining three symmetry-equivalent $C_{2}$ axes pass through the midpoints of opposite bonds, and are therefore less preferred according to rule Fu-3.1.2. Either one of the former $C_{2}$ axes can be used as the reference axis. Numbering can start in either one of the symmetry-equivalent hexagons. Because of symmetry, the atoms of this ring form two sets of atoms, indicated as $\mathbf{a} / \mathbf{a}^{\prime} / \mathbf{a} " / \mathbf{a}^{\prime \prime}$ and $\mathbf{b} / \mathbf{b}^{\prime}$. There are three pathways to be evaluated: $\mathbf{a}$ to $\mathbf{b}^{\prime}$ to $\mathbf{a}^{\prime \prime}$; $\mathbf{a}$ to $\mathbf{a}^{\prime}$ to $\mathbf{b}$; and $\mathbf{b}^{\prime}$ to $\mathbf{a}^{\prime \prime}$ to $\mathbf{a}^{\prime \prime}$. Of these, the $\mathbf{b}^{\prime}$ to $\mathbf{a}^{\prime \prime}$ to $\mathbf{a}$ " pathway does not lead to a contiguous numbering, whereas the other two do. The numberings are shown in Fig. 14b (a to b' to $\mathbf{a}^{\prime \prime \prime}$ ) and in Fig. 14c (a to $\mathbf{a}^{\prime}$ to $\mathbf{b}$ ). Since both terminate at an atom belonging to the ring intersected by the axis, a selection cannot be made on the basis of rule Fu-3.1.3. However, inspection of the atom-ranking according to rule Fu-3.1.4 shows that the numbering shown in Fig. 14b is preferred because it features a 6,6,6 rather than a $6,6,5$ atom at position 2 . 

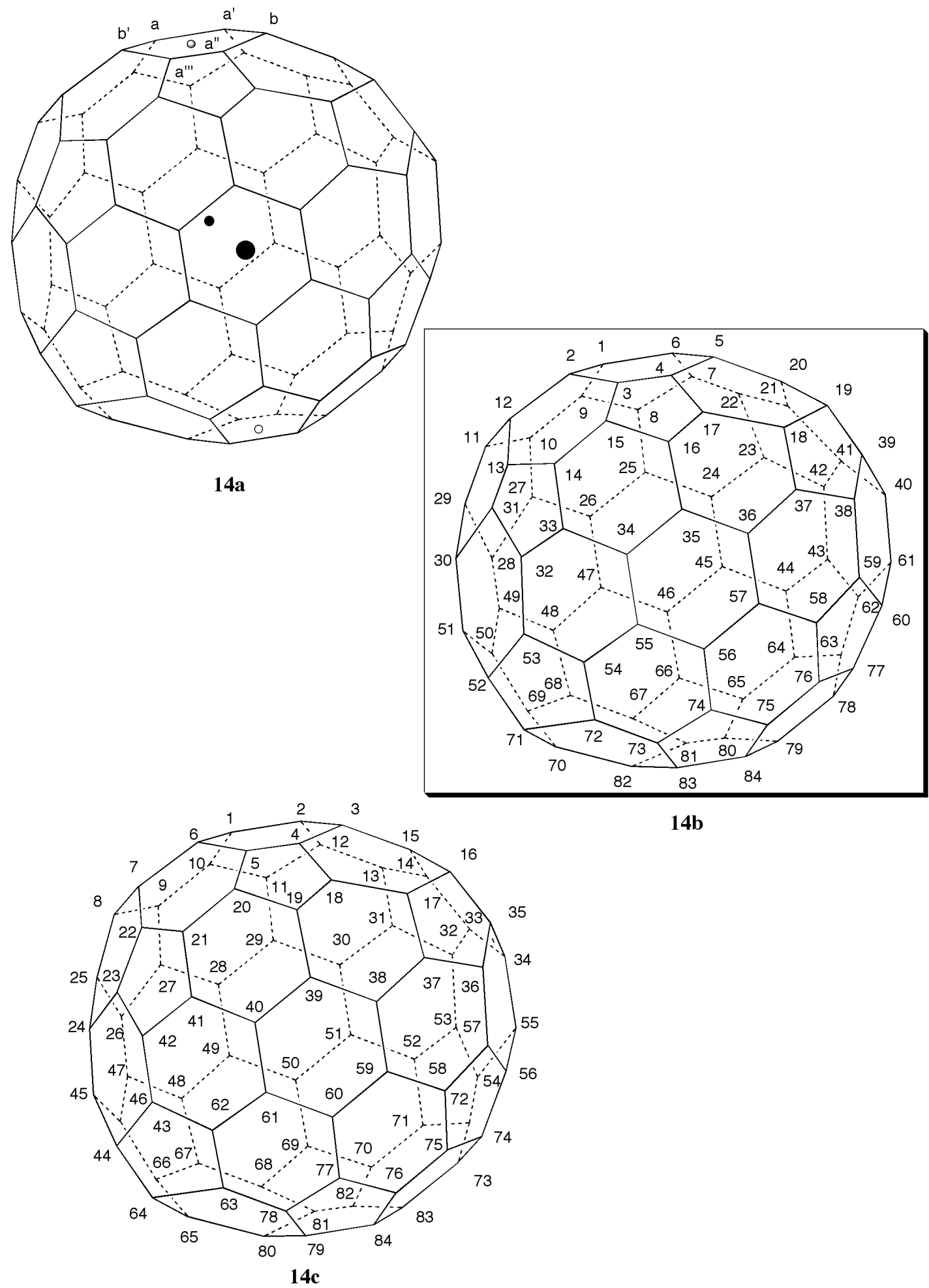

$14 b$

Fig. 14 Systematic numbering for $\left(\mathrm{C}_{84}-D_{6 \mathrm{~h}}\right)[5,6]$ fullerene. 


\subsection{Fullerenes having at least one symmetry axis $\left(C_{n}, n>1\right)$ but no contiguous helical pathway determined on the basis of rules Fu-3.1.1 to Fu-3.1.4}

Fullerenes with at least one symmetry axis $C_{\mathrm{n}}, \mathrm{n}>1$ exist for which a contiguous helical pathway for numbering cannot be found using rules Fu-3.1.1 to Fu-3.1.4. The problem of numbering discountiguity in these fullerenes has already been tackled by previous publications [1,3], and solutions were proposed using different and sometimes unclearly established procedures. To devise unique numbering for these fullerenes in a clear and relatively straightforward way, it was decided to establish a modified set of rules adopting an approach similar to that used in the case of fullerenes with contiguous numbering.

If no contiguous helical numbering can be found in a fullerene with at least one rotational axis of symmetry $C_{\mathrm{n}}, \mathrm{n}>1$ by application of rules $\mathbf{F u - 3 . 1 . 1}$ to $\mathbf{F u - 3 . 1 . 4}$, discontiguous numberings made up of two or more contiguous segments are examined, with the first of their segments being built around a proper rotation axis in complete analogy to the procedure employed in rule Fu-3.1 to determine a contiguous pathway. If a preferred first segment is obtained based on rules Fu-3.2.1 to Fu-3.2.3 (see below), its follow-up segment(s) is (are) constructed based on rule Fu-3.2.4 (see below) to complete the preferred numbering. If there is still a choice after comparison of different first segments, subsequent segments are constructed (rule Fu-3.2.4) and compared (rules Fu-3.2.1 to Fu-3.2.3) at each level until a preference becomes apparent.

Rule Fu-3.2.1 (cf. Fu-3.1.1):

a. The $n^{\text {th }}$ discontiguity must occur as late as possible in the numbering.

b. Among different pathways having the $n^{\text {th }}$ discontiguity at the same highest locant, the preferred numbering pathway is built around the proper rotation axis of higher order.

Rule Fu-3.2.2 (cf. Fu-3.1.2): If there is a choice among reference axes of the same order or between the end of a reference axis to begin the numbering, rule Fu-3.1.2 is applied.

Rule Fu-3.2.3 (cf. Fu-3.1.4; for practical reasons, no rules analogous to rule $\mathbf{F u}-\mathbf{3 . 1 . 3}$ are applied to segments of discontiguous numberings): When there is still a choice, the $n^{\text {th }}$ segments are compared according to rule $\mathbf{F u - 3 . 1 . 4 . ~}$

Once a preferred segment is identified based on rules $\mathbf{F u - 3 . 2 . 1}$ to $\mathbf{F u - 3 . 2 . 3 , ~ t h e ~ n u m b e r i n g ~ o f ~ t h e ~}$ fullerene is completed by construction of the subsequent segment(s). The starting point of a new segment is defined according to rule Fu-3.2.4a (see below), and numbering within a segment is continued according to rule $\mathbf{F u - 3 . 2 . 4 b}$.

Rule Fu-3.2.4: After each discontiguity,

a. the next atom to be numbered must be directly connected to one of the previously numbered atoms; among the latter, the atom with the highest locant is preferred;

b. numbering continues by the shortest path, in terms of number of bonds, to reach an unnumbered atom that is directly connected to a numbered atom with the lowest locant already assigned. (In this way, the sense of the numbering helix should be maintained, and its pitch kept as small as possible.)

Some examples describing the discontiguous numbering of axial fullerenes assigned on the basis of these rules are reported below.

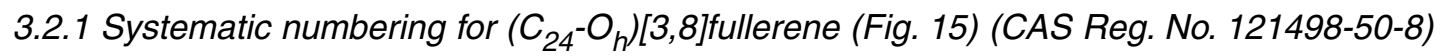
This fullerene, made up only of 8,8,3 atoms, possesses three symmetry-equivalent $C_{4}$ axes (Fig. 15a), four symmetry-equivalent $C_{3}$ axes (Fig. 15c), and six symmetry-equivalent $C_{2}$ axes (these axes join midpoints of opposite 8,8 bonds). When one of the $C_{4}$ axes is used as the reference axis, a contiguous pathway cannot be found starting along the bond shared by two eight-membered rings. In this case (Fig. 15a), the numbering becomes discontiguous at position 14. The numbering in Fig. 15b, obtained again using one of the $C_{4}$ axes, but starting along the bond shared by an eight- and a three-membered 


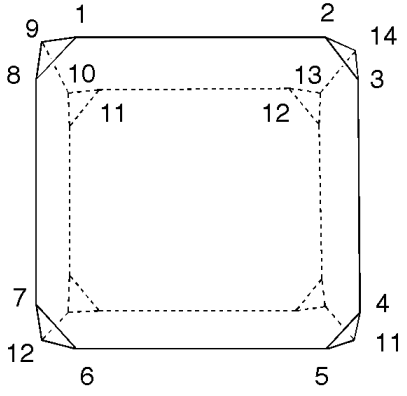

$15 a$

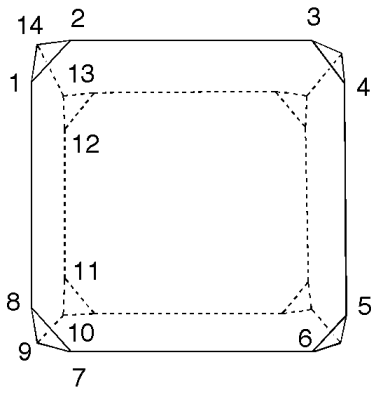

$15 b$

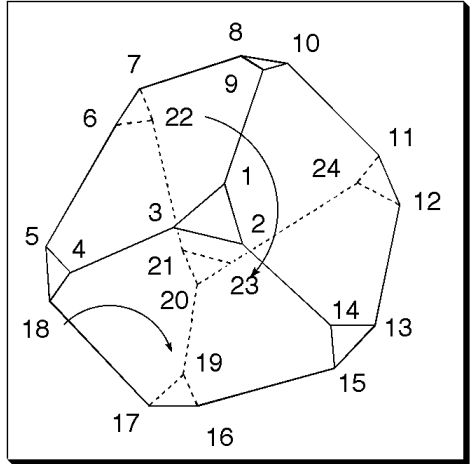

$15 \mathrm{c}$

Fig. 15 Systematic numbering for $\left(\mathrm{C}_{24^{-}} O_{\mathrm{h}}\right)[3,8]$ fullerene.

ring also becomes discontiguous at position 14. In Fig. 15c, the numbering obtained using one of the $C_{3}$ axes as the reference axis is shown. In this case, numbering becomes discontiguous at position 18 . The numbering obtained using a $C_{2}$ axis as the reference axis becomes discontiguous at a position with a locant lower than both 14 and 18. According to rule Fu-3.2.1a, the numbering in Fig. 15c is preferred because the discontiguity occurs at a higher locant. According to rule Fu-3.2.4a, the numbering proceeds to position 19, which is connected to the numbered atom with the highest locant within reach (17). Within the second segment, numbering proceeds by the shortest path to position 22 which is connected to the numbered atom with the lowest locant within reach (6) (rule Fu-3.2.4b). After another discontiguity, a third segment starts at position 23 (connected to atom number 21 according to rule Fu-3.2.4a) and ends with the last unnumbered, neighboring atom (24).

\subsubsection{Systematic numbering for $\left(C_{48}-O_{h}\right)[4,6,8] f u l l e r e n e$ (Fig. 16) [(CAS Reg. No. 145985-79-1, as fulleride(2-)]}

This fullerene, made up only of 8,6,4 atoms, possesses three symmetry-equivalent $C_{4}$ axes (Fig. 16a), four symmetry-equivalent $C_{3}$ axes (Fig. 16b), and six symmetry-equivalent $C_{2}$ axes (not shown: the axes pass through the midpoints of opposite four-membered rings). When one of the $C_{4}$ axes is used as the reference axis (Fig. 16a) and the pathway begins along a bond shared by an eight- and a six-membered ring, the numbering becomes discontiguous at position 40 . When, using the same axis, a bond shared by an eight- and a four-membered ring is selected to begin the numbering (namely, C-2 to C-3 in Fig. 16a), the discontiguity occurs at position 24. The numbering obtained using one of the $C_{3}$ axes as the reference axis and starting along a bond shared by an eight- and a six-membered ring is shown in Fig. 16b. In this case, the discontiguity occurs at position 42. Using the same axis and starting along a bond shared by a six- and a four- membered ring (namely, C-2 to C-3 in Fig. 16b), the numbering becomes discontiguous at position 24 . The use of a $C_{2}$ axis leads to numberings discontiguous at positions with lower locants. Therefore, on the basis of Fu-3.2.1a, the numbering shown in Fig. 16b is preferred. According to Fu-3.2.4a, the numbering resumes at position 43, which is connected to 41 , and then continues contiguously to position 48 according to $\mathbf{F u - 3 . 2 . 4 b .}$ 

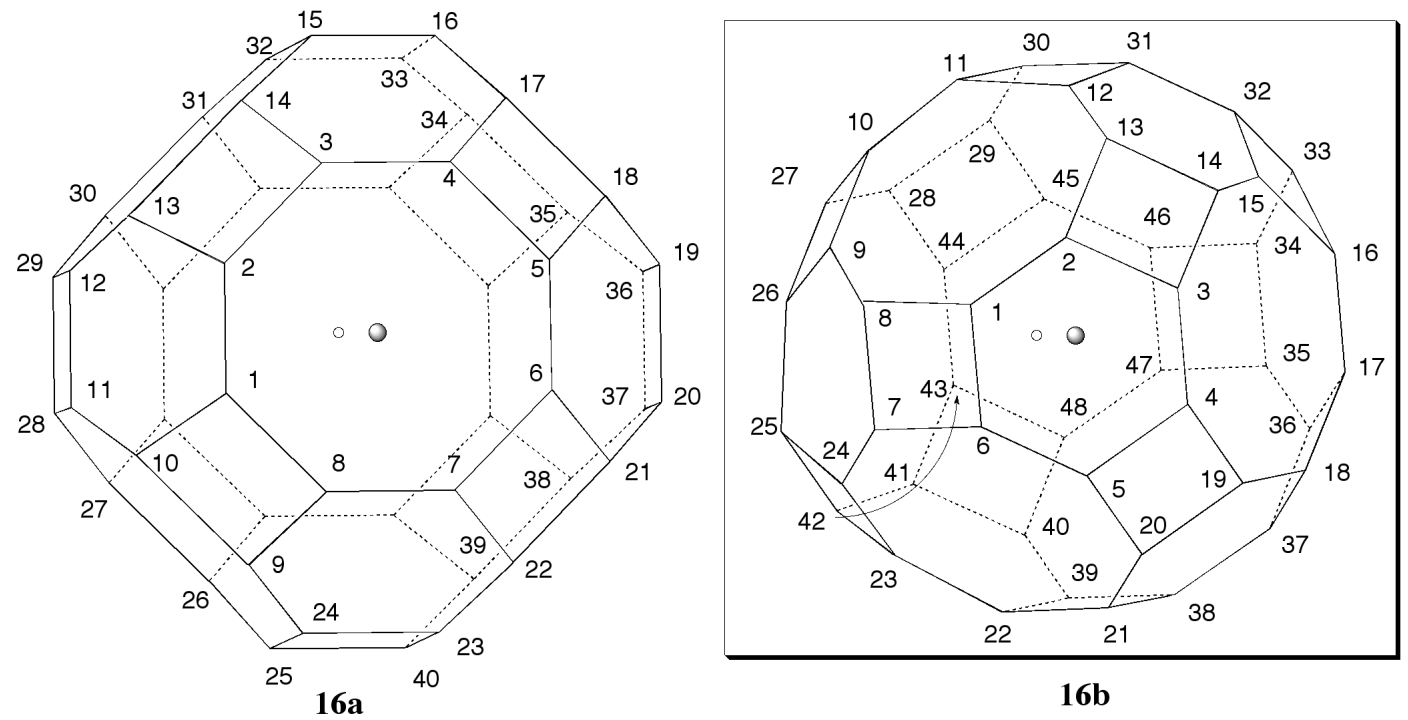

Fig. 16 Systematic numbering for $\left(\mathrm{C}_{48}{ }^{-} O_{\mathrm{h}}\right)[4,6,8]$ fullerene.

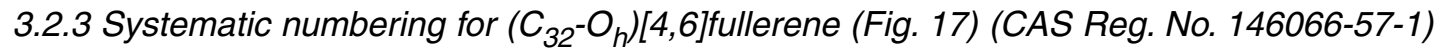
This fullerene features three symmetry-equivalent $C_{4}$ axes (Fig. 17a), four symmetry-equivalent $C_{3}$ axes (not shown: they pass through opposite atoms at the intersection of three six-membered rings), and six symmetry-equivalent $C_{2}$ axes (Fig. 17b). When one of the $C_{4}$ axes is used as the reference axis (Fig. 17a), there is only one possible pathway, and the numbering becomes discontiguous at position 28. Using one of the $C_{3}$ axes, the only possible pathway leads to a numbering discontiguous at position 25 . Using one of the $C_{2}$ axes as the reference axis, there are three possible pathways that lead to numberings discontiguous at positions 25, 26, and 28, respectively. The latter is shown in Fig. 17b. Thus, two discontiguous numberings with a first segment of equal length are found. However, according to Fu-3.2.1b, the numbering reported in Fig. 17a is preferred, because its pathway uses the highest-order

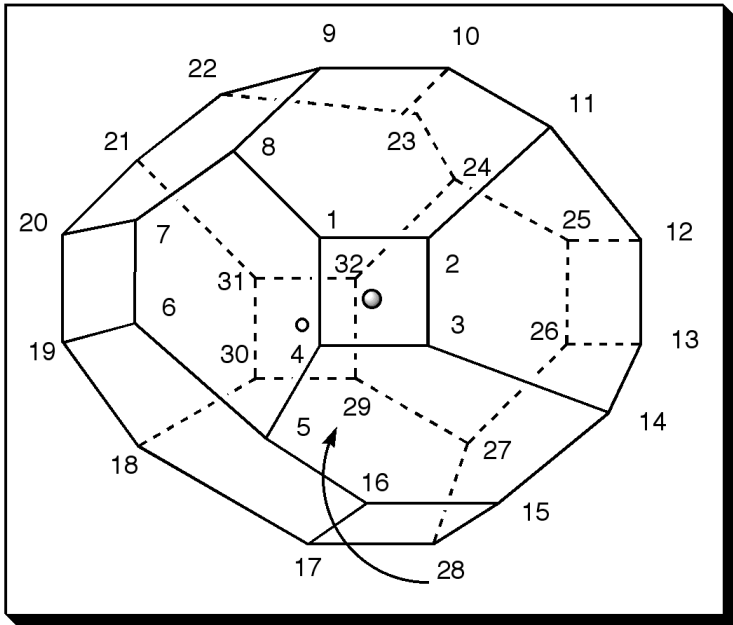

$17 \mathbf{a}$

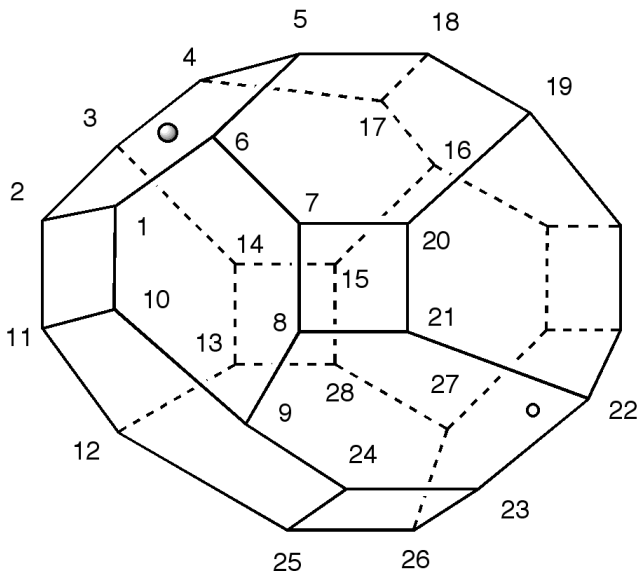

$17 \mathbf{b}$

Fig. 17 Systematic numbering for $\left(\mathrm{C}_{32}-O_{\mathrm{h}}\right)[4,6]$ fullerene. 
axis as the reference axis. From position 28, the numbering resumes as shown in Fig. 17a (Fu-3.2.4a) to reach position $32(\mathbf{F u}-\mathbf{3 . 2 . 4 b})$ without further discontiguity.

\subsubsection{Systematic numbering for $\left(C_{34}-C_{2 v}\right)[4,6]$ fullerene (Fig. 18)}

This fullerene contains one $C_{2}$ axis intersecting the midpoint of a six-membered ring at the near end and of a 6,6 bond at the far end (Fig. 18a). The six atoms of the ring form two sets of symmetry-equivalent atoms: $\mathbf{a} / \mathbf{a}^{\prime}$ and $\mathbf{b} / \mathbf{b}^{\prime} / \mathbf{b}^{\prime \prime} / \mathbf{b}^{\prime \prime}$. Three pathways must be considered: $\mathbf{a}$ to $\mathbf{b}$ to $\mathbf{b}^{\prime} ; \mathbf{b}$ to $\mathbf{b}^{\prime}$ to $\mathbf{a}^{\prime}$; and $\mathbf{b}$ to $\mathbf{a}$ to $\mathbf{b}^{\prime \prime}$. These lead to discontiguous numberings terminating at positions 20, 28 (Fig. 18b), and 25, respectively. The two atoms $\mathbf{z}$ and $\mathbf{z}^{\prime}$ (Fig. 18a) connected by the bond at the far end of the axis are symmetry-equivalent. The pathway from $\mathbf{z}$ becomes discontiguous at position 32 (Fig 18c), and is therefore preferred on the basis of rule Fu-3.2.1a. The completion of this numbering according to rule Fu-3.2.4 is shown in Fig. 18c.
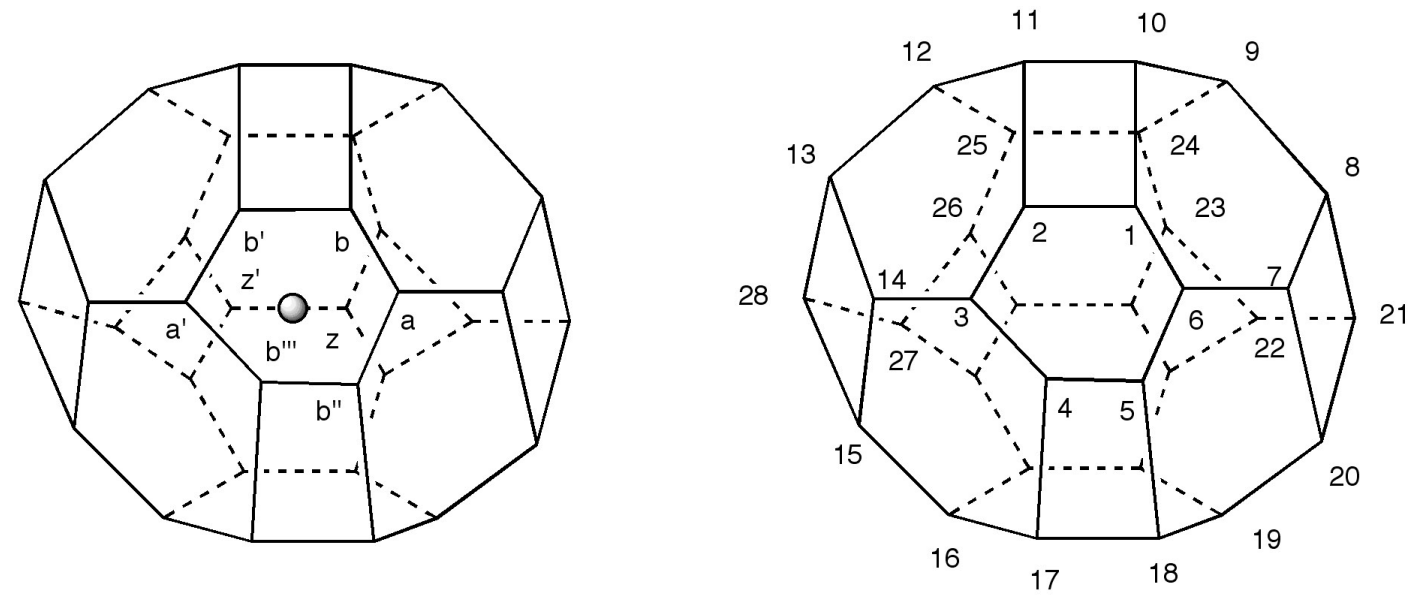

$18 \mathbf{a}$ $18 b$

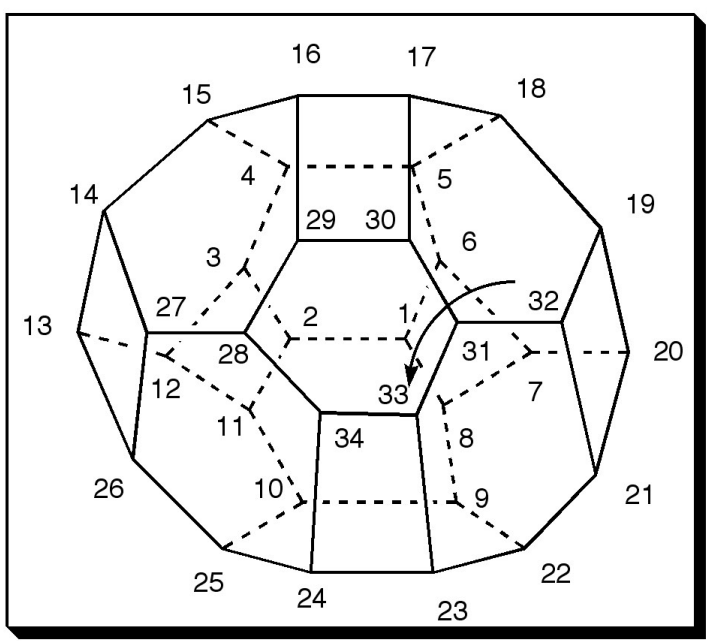

$18 \mathrm{c}$

Fig. 18 Systematic numbering for $\left(\mathrm{C}_{34}{ }^{-} C_{2 \mathrm{v}}\right)[4,6]$ fullerene. 


\subsubsection{Systematic numbering for $\left(C_{60}-D_{6 h}\right)[5,6]$ fullerene (Fig. 19)}

This fullerene features one $C_{6}$ axis, passing through the midpoints of opposite hexagons (centers of coronene substructures), three symmetry-equivalent $C_{2}$ axes, also passing through the midpoints of opposite hexagons, and three symmetry-equivalent $C_{2}$ axes, passing through the midpoint of opposite bonds (Fig. 19a). Starting from any of the six symmetry-equivalent atoms a of the six-membered ring intersected by the $C_{6}$ axis does not lead to a contiguous helical pathway. The numbering built around the $C_{6}$ axis (Fig. 19b) becomes discontiguous at position 52. Three pathways must be considered for beginning the numbering based on one of the $C_{2}$ axes passing through opposite hexagons: $\mathbf{c}$ to $\mathbf{c}^{\prime}$ to $\mathbf{b}^{\prime}$; $\mathbf{c}^{\prime}$ to $\mathbf{b}^{\prime}$ to $\mathbf{c}^{\prime \prime}$; and $\mathbf{b}^{\prime}$ to $\mathbf{c}^{\prime \prime}$ to $\mathbf{c}^{\prime \prime}$. These pathways lead to numberings discontiguous at position 58 (Fig. 19c), 56 (not shown), and 58 (Fig. 19d), respectively. Finally, one pathway can also be constructed around one of the remaining $C_{2}$ axes passing through the midpoints of opposite bonds. This pathway results in the numbering shown in Fig. 19e that is discontiguous at position 58. To select among the three numberings having the discontiguity at the same higher position and built around axes of the same order (Figs. 19c, 19d, and 19e), rule Fu-3.2.2 is first applied. The two numberings beginning in a ring (Figs. $19 \mathrm{c}$ and $19 \mathrm{~d}$ ) are preferred over the one beginning at a bond bisected by a $C_{2}$ axis (Fig. 19e). Furthermore, according to rule $\mathbf{F u - 3 . 2 . 3}$, the numbering beginning with a 6,6,5 atom (Fig. 19c) is preferred over the one beginning with a 6,5,5 atom (Fig. 19d). The completion of the numbering of Fig. 19c is straightforward on the basis of rule $\mathbf{F u - 3 . 2 . 4}$. 


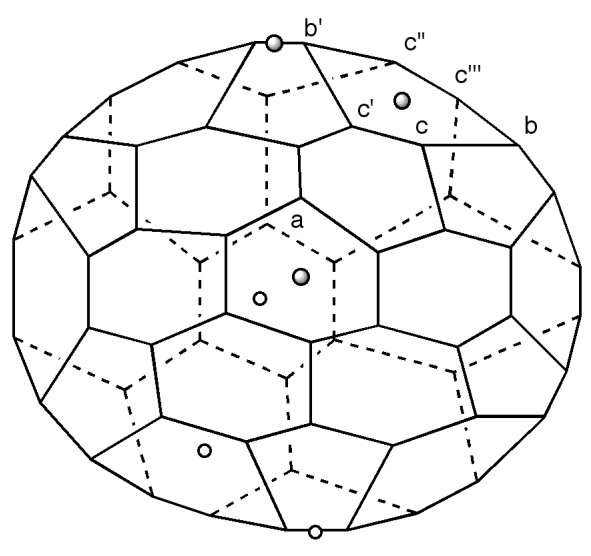

$19 \mathbf{a}$

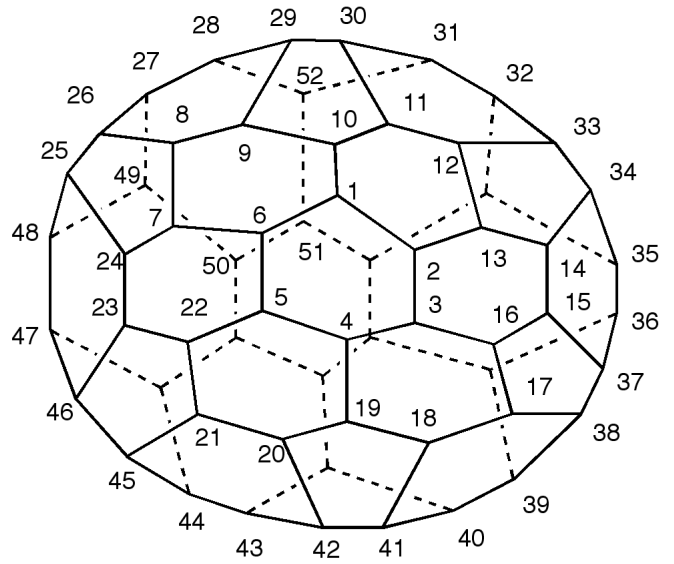

$19 \mathrm{~b}$
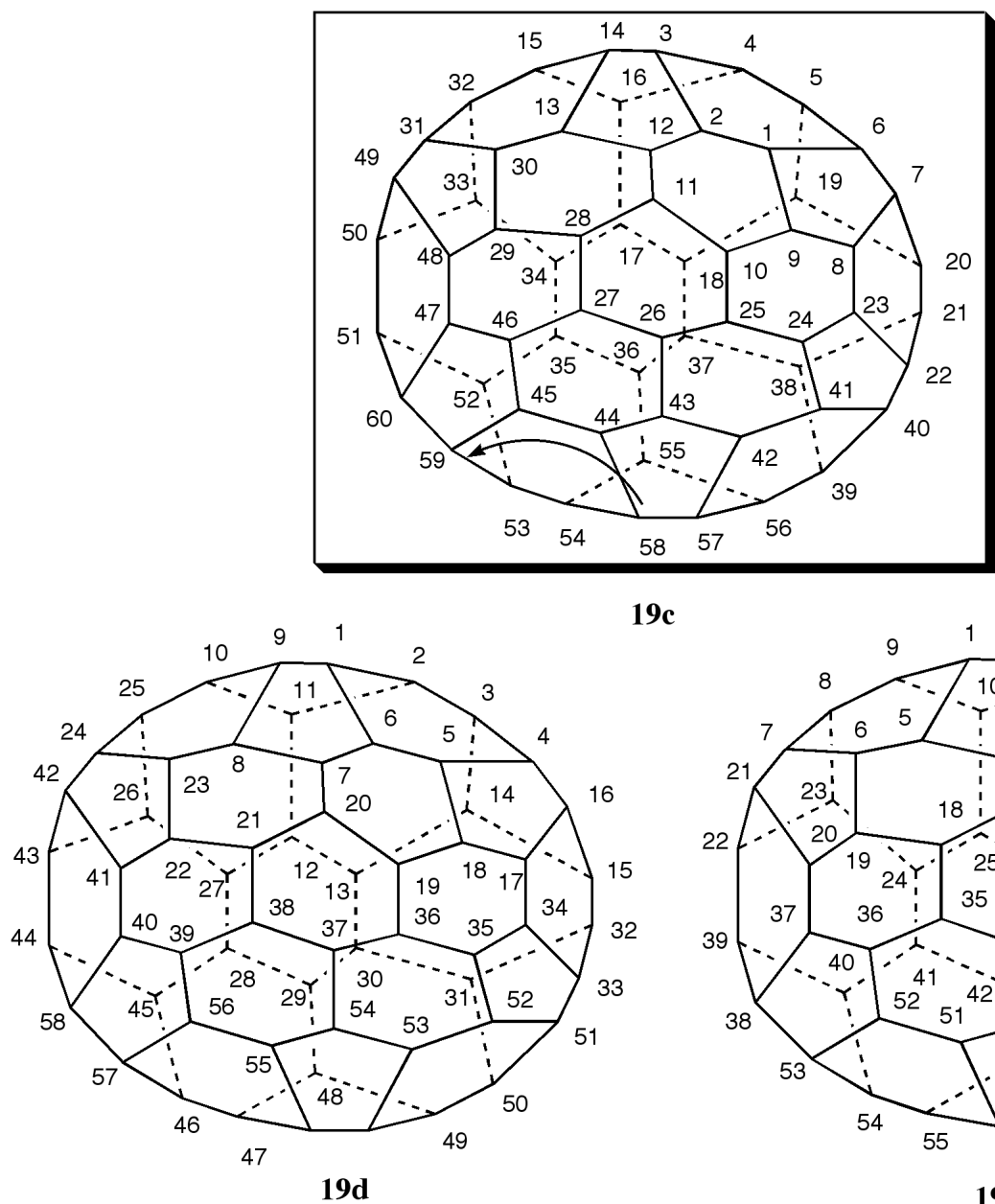

$19 c$

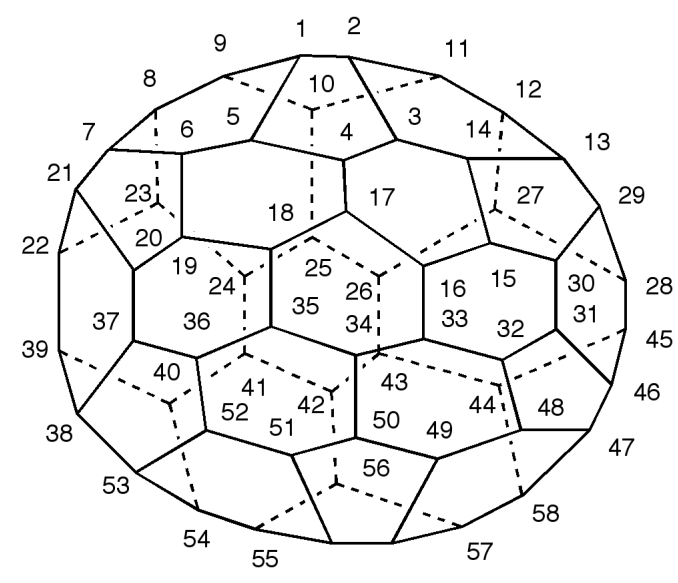

$19 e$

Fig. 19 Systematic numbering for $\left(\mathrm{C}_{60}-D_{6 \mathrm{~h}}\right)[5,6]$ fullerene. 


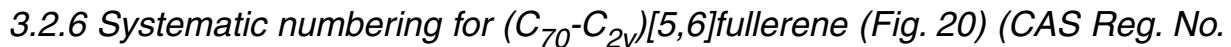 163479-23-0)}

This fullerene contains a single $C_{2}$ axis connecting the center of a six-membered ring at the near end and the midpoint of a bond at the far end, as shown in Fig. 20a. For symmetry reasons, three pathways must be considered in the ring: $\mathbf{a}$ to $\mathbf{b}$ to $\mathbf{a} " ; \mathbf{a}$ to $\mathbf{a}^{\prime}$ to $\mathbf{b}^{\prime}$; and $\mathbf{b}$ to $\mathbf{a}$ to $\mathbf{a}^{\prime}$. They lead to numberings that become discontiguous at positions 54, 55, and 59, respectively (not shown). The only pathway that starts from the bond [( $\left(\mathbf{z}\right.$ to $\left.\mathbf{z}^{\prime}\right)$ is equivalent to ( $\mathbf{z}^{\prime}$ to $\left.\mathbf{z}\right)$, Fig. 20a] leads to the numbering shown in Fig. 20b; since this becomes discontiguous only at position 63, it is preferred on the basis of rule Fu-3.2.1a. The completion of the numbering represents an interesting example of the application of rule Fu-3.2.4 (see arrows in Fig. 20b). From position 63, a discontiguous step leads to atom 64, which is bound to atom 62 (rule Fu-3.2.4a). Moving on from atom 64, there are two possibilities, but the one going to the atom connected to atom 44 is preferred by rule Fu-3.2.4b. Atom 66 is selected again on the basis of rules Fu-3.2.4a, and Fu-3.2.4b allows the numbering to reach atoms 67 and 68. A final application of rule Fu-3.2.4 allows the last segment of the numbering to be completed.

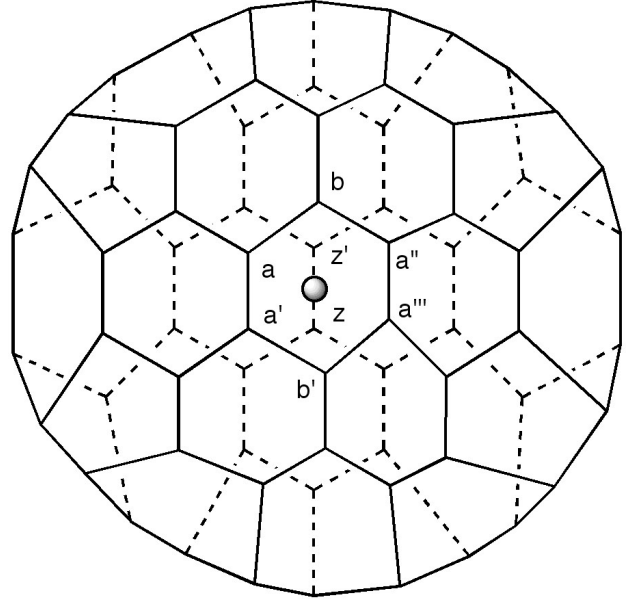

20a

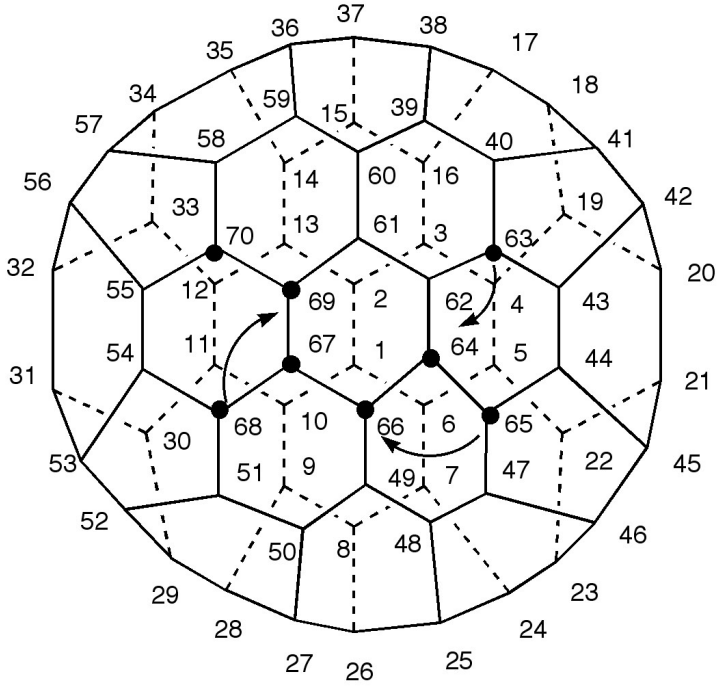

$20 \mathrm{~b}$

Fig. 20 Systematic numbering for $\left(\mathrm{C}_{70}-C_{2 \mathrm{v}}\right)[5,6]$ fullerene.

\subsubsection{Systematic numbering for $\left(C_{80^{-}} I_{h}\right)[5,6]$ fullerene (Fig. 21) (Atlas Ref. No. 80:7; CAS Reg. No. 133947-14-5)}

This fullerene features six symmetry-equivalent $C_{5}$ axes (connecting midpoints of opposite five-membered rings) ten symmetry-equivalent $C_{3}$ axes (connecting atoms at the intersection of three six-membered rings), and fifteen symmetry-equivalent $C_{2}$ axes (passing through the midpoints of opposite hexagons). These axes are shown in Fig. 21a. When one of the $C_{5}$ axes is used as the reference axis, the pathway can begin at any one of the five symmetry-equivalent atoms of the pentagon (indicated as $\mathbf{a}$ in Fig. 21a); the numbering (shown in Fig. 21b) becomes discontiguous at position 75 . When one of the $C_{3}$ axes is used as the reference axis, only one pathway from atom $\mathbf{b}$ must be considered; this pathway becomes discontiguous at position 66, and is therefore discarded (rule Fu-3.2.3). When one of the $C_{2}$ axes is used as the reference axis, there are three pathways to be considered: $\mathbf{c}$ to $\mathbf{d}$ to $\mathbf{e} ; \mathbf{d}$ to $\mathbf{e}$ to $\mathbf{c} ; \mathbf{e}$ to $\mathbf{c}^{\prime}$ to $\mathbf{d}^{\prime}$. One of these leads to the numbering becoming discontiguous at position 75 (not shown); the others lead to numberings in which the discontiguities occur at positions 72 and 66. According to rule 


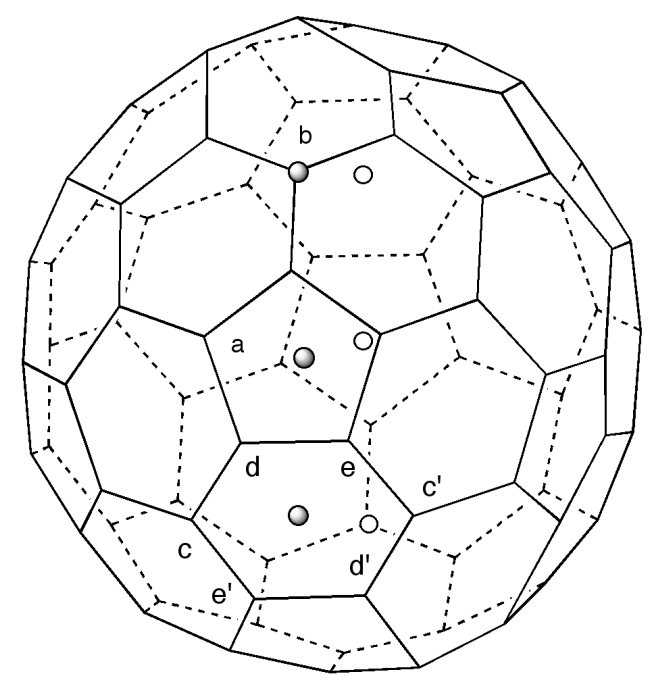

$21 \mathbf{a}$

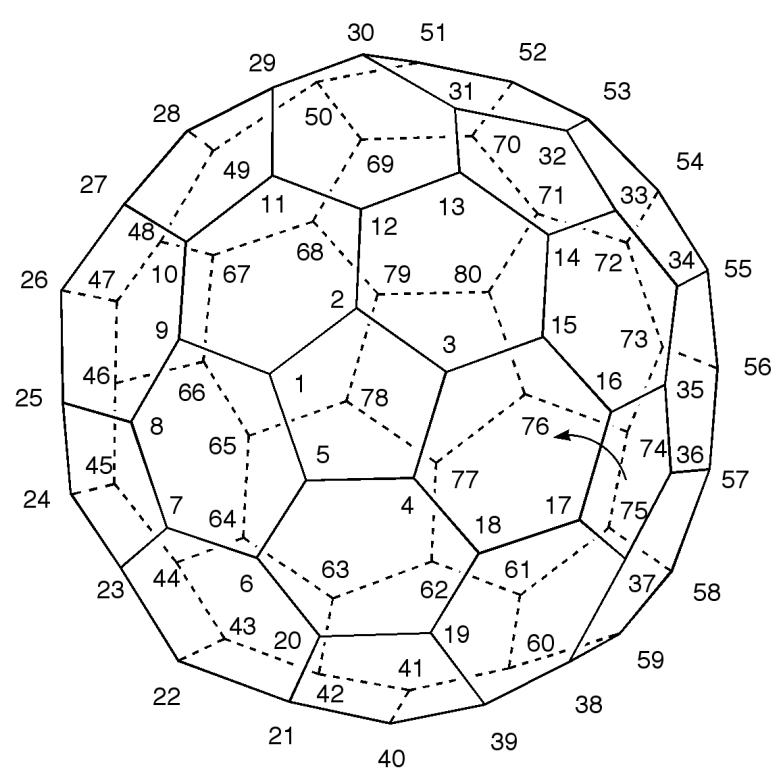

$21 b$

Fig. 21 Systematic numbering for $\left(\mathrm{C}_{80}-I_{\mathrm{h}}\right)[5,6]$ fullerene.

Fu-3.2.1, the three numberings built around the $C_{2}$ axis are discarded. The correct numbering is, therefore, that shown in Fig. 21b. The completion of the numbering by application of rule Fu-3.2.4 is straightforward.

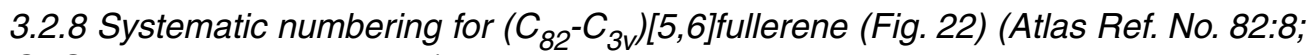 CAS Reg. No. 147230-79-3)}

This fullerene features one $C_{3}$ axis connecting the center of a six-membered ring at the near end and an atom at the intersection of three six-membered rings at the far end (Fig. 22a). For symmetry reasons, two pathways starting in the ring must be considered: a to $\mathbf{a}^{\prime}$ to $\mathbf{a}$ " and $\mathbf{a}$ ' to $\mathbf{a}$ " to $\mathbf{a}^{\prime \prime}$. They both lead to numberings that become discontiguous at position 81, as shown in Figs. 22b and 22c, respectively. The pathway starting from the atom $\mathbf{z}$ at the far end of the axis leads to a numbering discontiguous at position 66 (not shown), that is discarded on the basis of rule Fu-3.2.1. Selection between the remaining two numberings is made on the basis of rule Fu-3.2.3. By comparing the ranking of the atoms of the two sequences (cf. rule Fu-3.1.4), it is found that the atom at position 8 of the numbering in Fig. 22b is a 6,6,6 atom, whereas the numbering shown in Fig. 22c features a 6,6,5 atom at the same position. The former is, therefore, preferred. The completion of the numbering is obvious, because the unassigned site is reduced to a final isolated unnumbered atom that receives the last number in the sequence. 

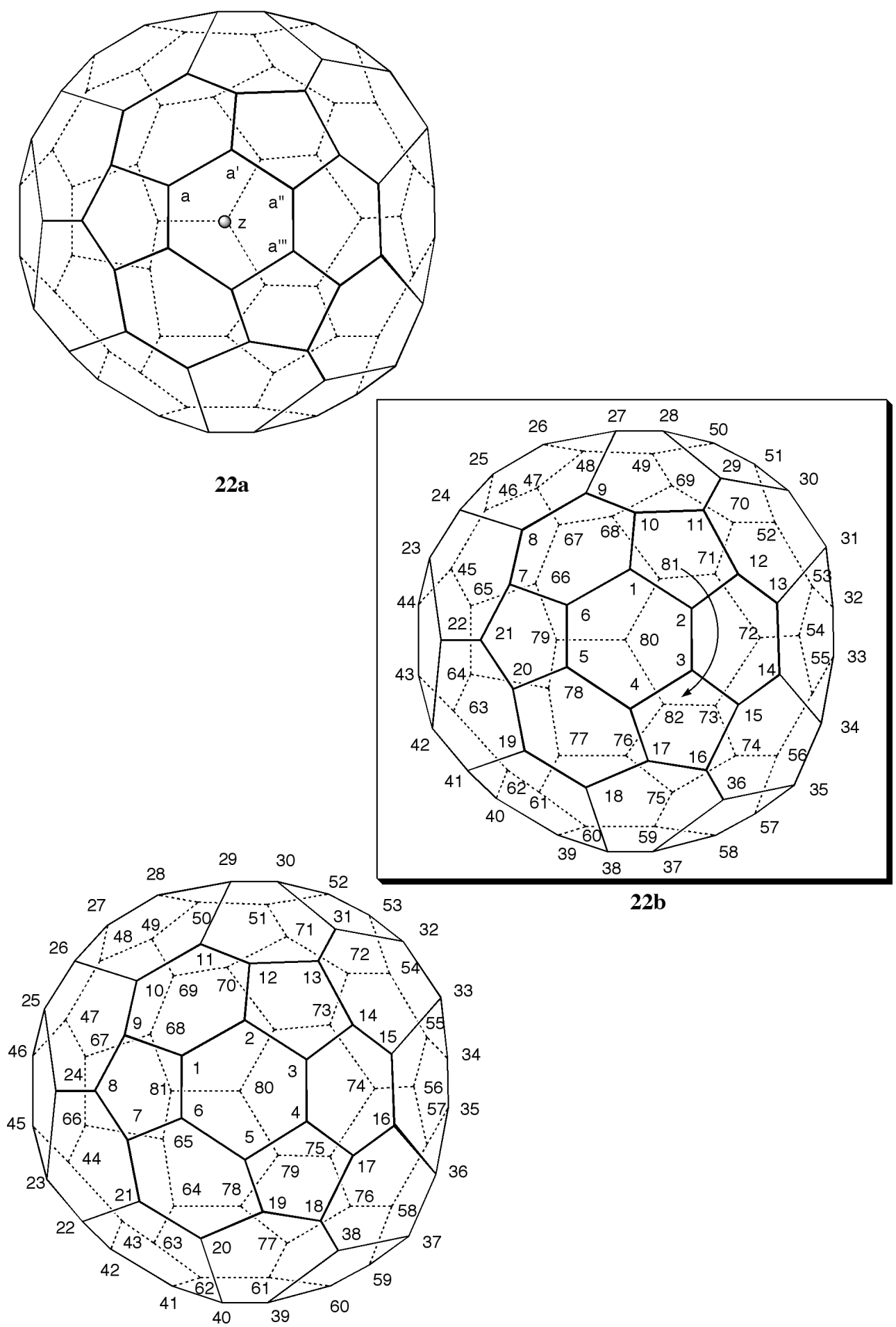

$22 c$

Fig. 22 Systematic numbering for $\left(\mathrm{C}_{82}-C_{3 \mathrm{v}}\right)[5,6]$ fullerene. 


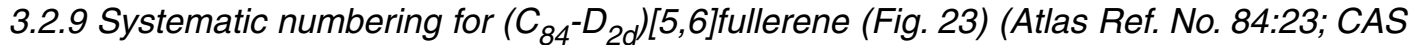
Reg. No. 145809-20-7)

This fullerene contains three $C_{2}$ axes, each intersecting midpoints of opposite bonds as shown in Fig. 23a. Since the axes have the same order, the numbering can start at any of the bonds bisected by the axes. Only three pathways must be evaluated: $\mathbf{a}$ to $\mathbf{a}$ ' to $\mathbf{d} ; \mathbf{b}$ to $\mathbf{b}^{\prime}$ to $\mathbf{e} ; \mathbf{c}$ to $\mathbf{c}^{\prime}$ to $\mathbf{f}$. None of these leads to a contiguous numbering: all of them are discontiguous at position 81 (Figs. 23b, 23c, and 23d). Exploration of the numberings according to rule Fu-3.2.3 shows that the numberings reported in Figs. $23 \mathrm{~b}$ and $23 \mathrm{~d}$ are preferred because they contain a 6,6,6 atom at position 15 . Further exploration

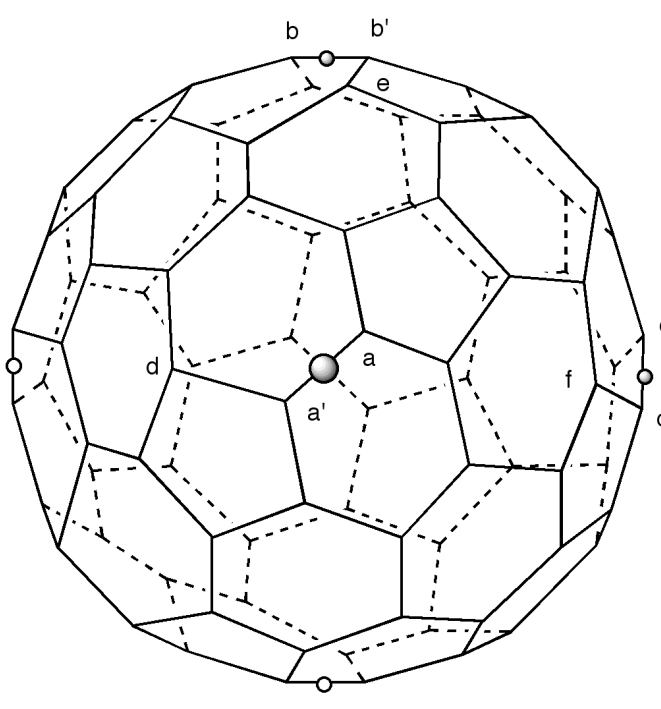

23a

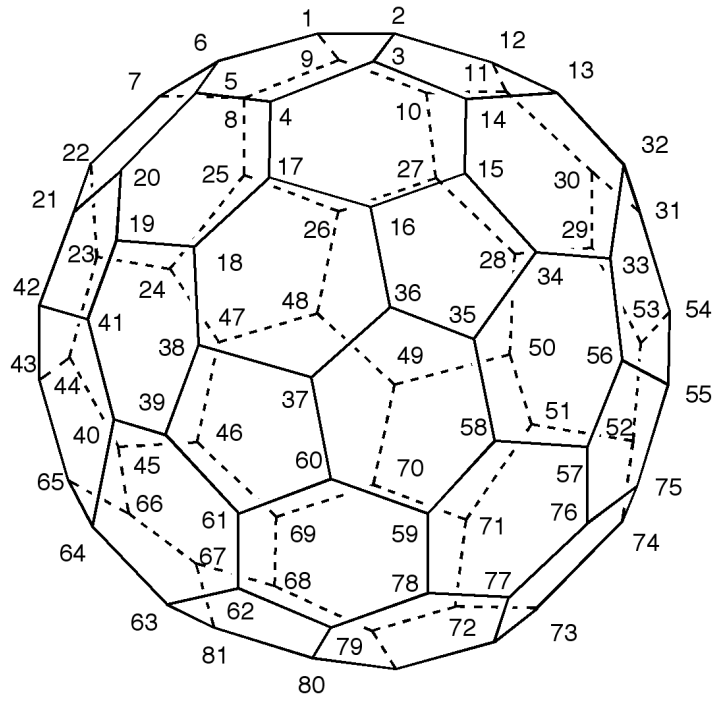

$23 c$

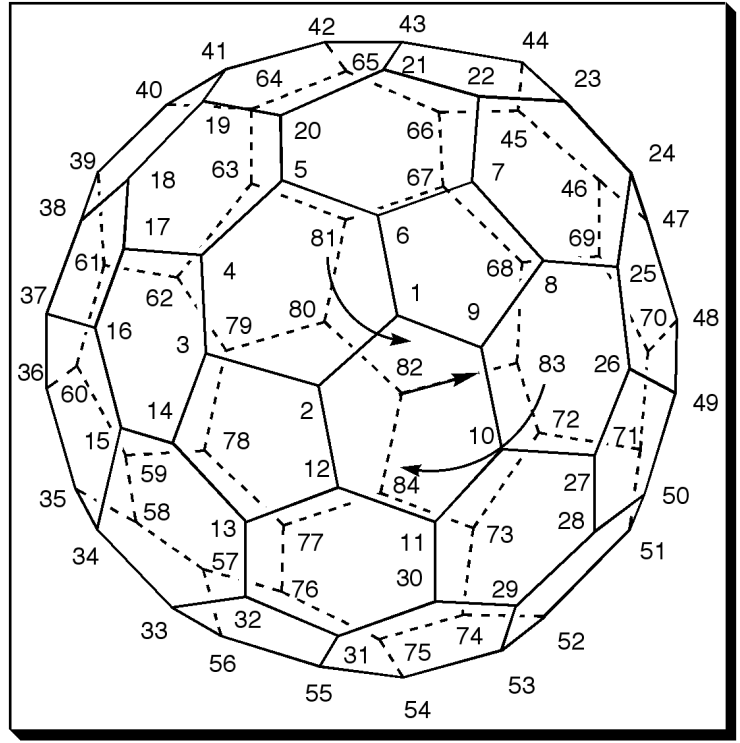

$23 b$

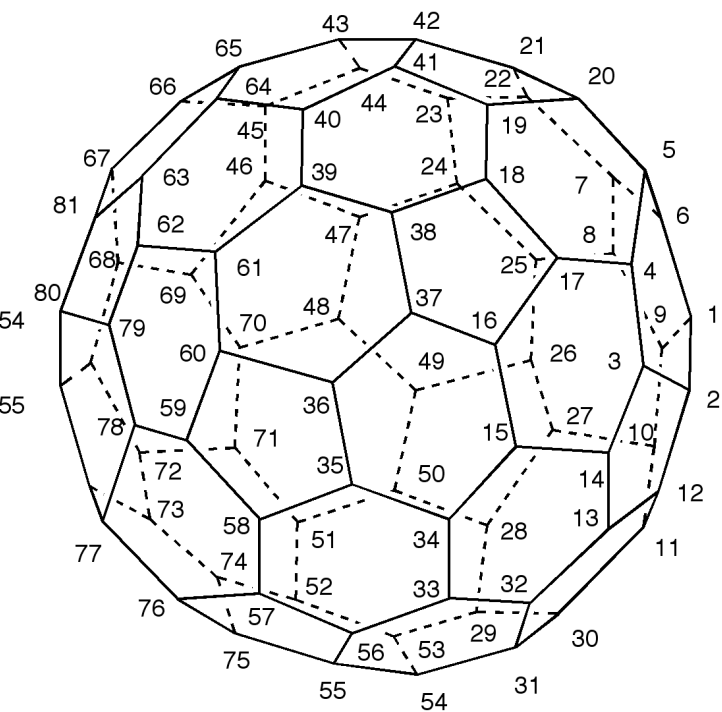

23d

Fig. 23 Systematic numbering for $\left(\mathrm{C}_{84}-D_{2 \mathrm{~d}}\right)[5,6]$ fullerene. 
shows that the numbering of Fig. 23b is preferred since it contains a 6,6,6 atom at position 19, whereas that of Fig. 23d contains a 6,6,5 atom at the same position. According to rule Fu-3.2.4, the completion of the numbering requires a discontiguous step to reach position 82 connected to the atom with the higher locant 80 (rule Fu-3.2.4a), a contiguous step to position 83 (rule Fu-3.2.4b), and a final discontiguous step to reach position 84 .

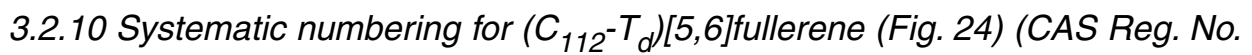 160763-20-2)}

This fullerene features four symmetry-equivalent $C_{3}$ axes connecting the midpoint of a six-membered ring on one side and an atom at the intersection of three six-membered rings on the other side (Fig. 24a); and three symmetry-equivalent $C_{2}$ axes connecting the midpoints of opposite six-membered rings (Fig. 24b). For symmetry reasons, there are two pathways to be considered starting from the hexagon bisected by the $C_{3}$ axis: from $\mathbf{a}$ and from $\mathbf{b}$. The former (shown in Fig. 24c) becomes discontiguous at position 111; the latter at position 93. The only pathway starting from the atom $\mathbf{z}$ (Fig. 24a) lying on the $C_{3}$ axis becomes discontiguous at position 72 . Using one of the $C_{2}$ axes as the reference axis, three clockwise pathways must be considered: from c, from d', and from d (Fig. 24b). They lead to numberings becoming discontiguous at positions 74,104 , and 107, respectively. Therefore, the numbering in Fig. 24c is preferred according to $\mathbf{F u - 3 . 2 . 1}$. 


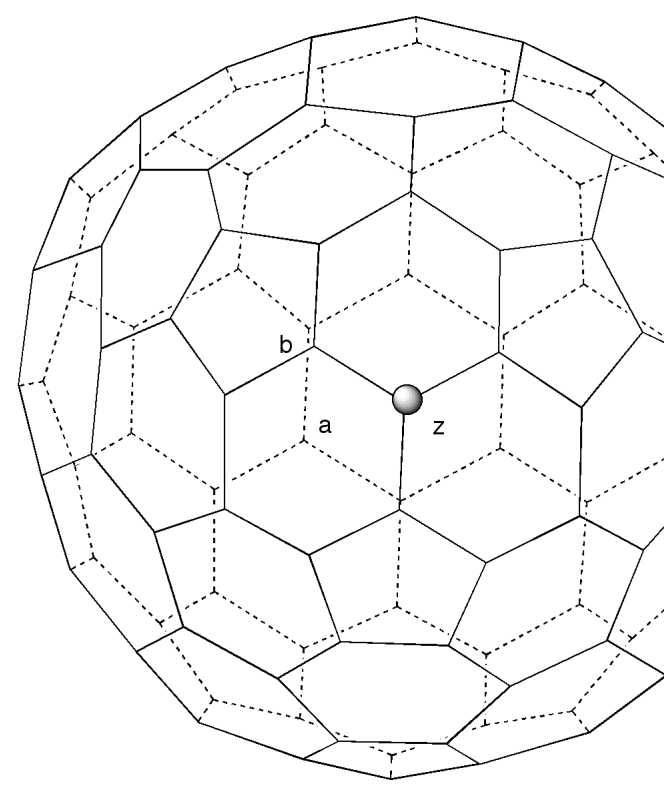

24a
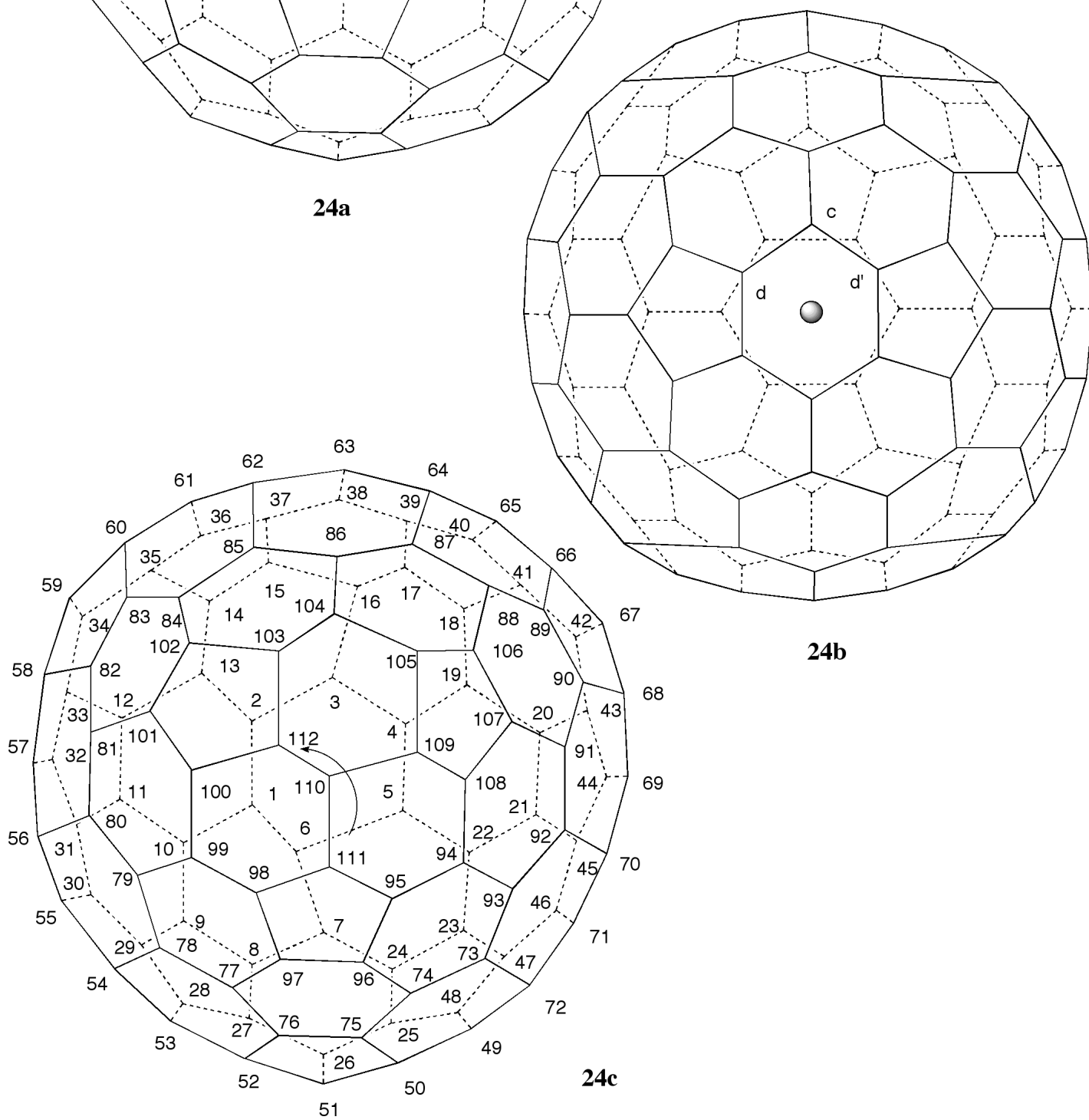

24b

Fig. 24 Systematic numbering for $\left(\mathrm{C}_{112}-T_{\mathrm{d}}\right)[5,6]$ fullerene. 


\subsubsection{Systematic numbering for $\left(C_{120^{-I}}\right)[4,6,10]$ fullerene (Fig. 25) (CAS Reg. No. 103598-39-6)}

This fullerene features six symmetry-equivalent $C_{5}$ axes, ten symmetry-equivalent $C_{3}$ axes, and fifteen symmetry-equivalent $C_{2}$ axes. The $C_{5}$ axes connect midpoints of opposite ten-membered rings; the $C_{3}$ axes connect midpoints of opposite six-membered rings; the $C_{2}$ axes connect midpoints of opposite four-membered rings (Fig. 25a). Even if all the atoms in this fullerene are symmetry-equivalent, different lettering is maintained for clarity. For symmetry reasons, there are two pathways to be considered in the ten-membered ring: a to $\mathbf{a}^{\prime \prime}$ and $\mathbf{a}^{\prime}$ to $\mathbf{a}$. Of these, the $\mathbf{a}$ to $\mathbf{a}$ " one leads to a numbering that becomes discontiguous at position 60 (Fig. 25b); the a' to a one leads to a numbering discontiguity at position 30 . For symmetry reasons, there are two pathways to be considered in the six-membered ring: $\mathbf{b}$ to $\mathbf{b}^{\prime}$ and $\mathbf{b}^{\prime}$ to $\mathbf{b}^{\prime \prime}$. Of these, the $\mathbf{b}$ to $\mathbf{b}^{\prime}$ pathway leads to a numbering that becomes discontiguous at position 30; the $\mathbf{b}^{\prime}$ to $\mathbf{b}^{\prime \prime}$ pathway leads to a numbering discontiguous at position 60 (Fig. 25c). For symmetry reasons, there are two pathways to be considered in the four-membered ring: $\mathbf{a}$ ' to $\mathbf{a}$ and $\mathbf{a}$ to $\mathbf{c}$. Of these, the a' to a pathway leads to a numbering that becomes discontiguous at position 32 ; the a to c pathway leads to a numbering discontiguity at position 36 . Selection between the numberings of Figs. $25 \mathrm{~b}$ and $25 \mathrm{c}$, that have the first discontiguity at the same position (60), can be made considering that the numbering of Fig. 25b is built around an axis of higher order (rule Fu-3.2.1b) and is therefore preferred. The completion of the preferred discontiguous numbering is made according to Fu-3.2.4. 


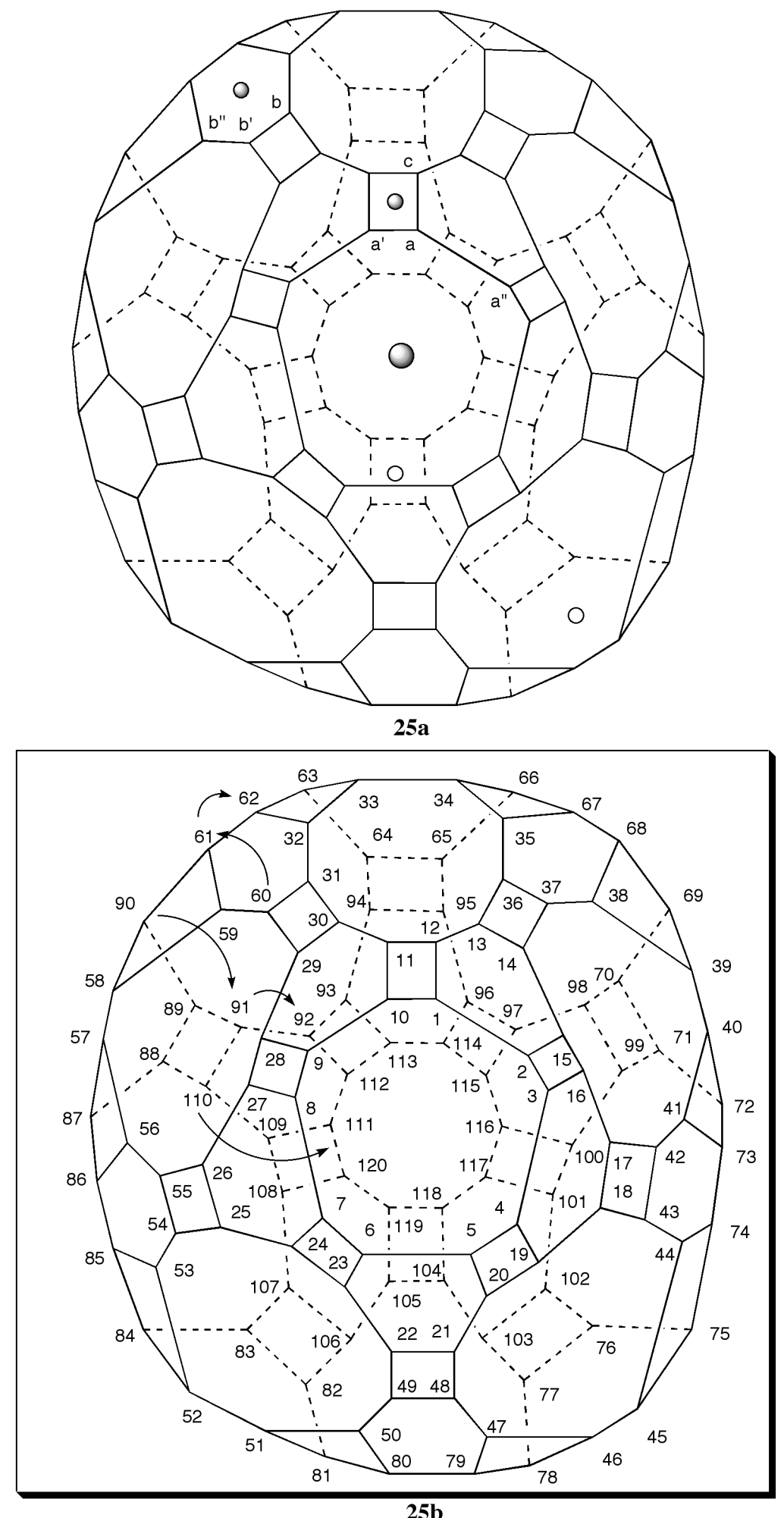

Fig. 25 Systematic numbering for $\left(\mathrm{C}_{120^{-}} \mathrm{I}_{\mathrm{h}}\right)[4,6,10]$ fullerene. 


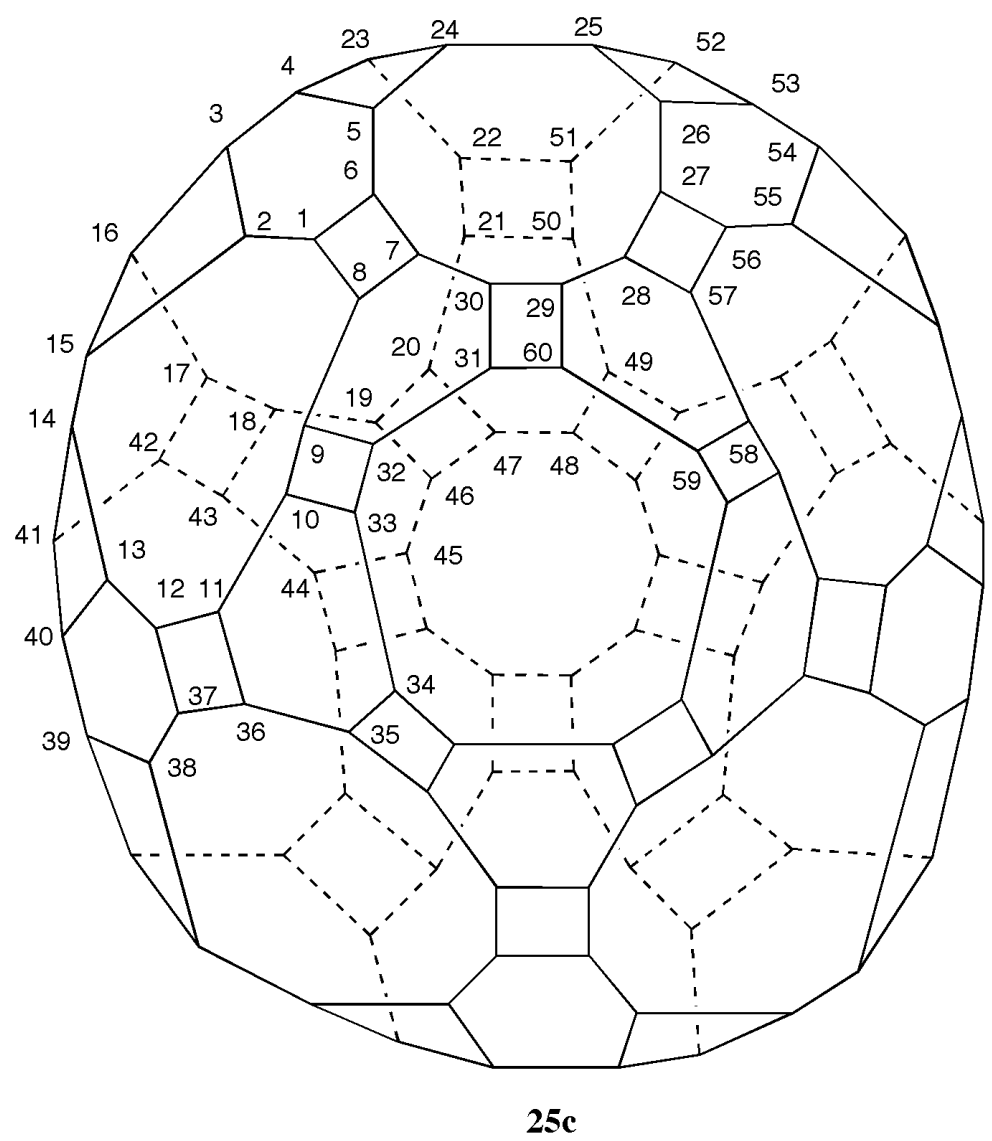

Fig. 25 (Continued).

\subsection{Fullerenes belonging to the $C_{\mathrm{s}}$ point group and having a contiguous helical numbering pathway according to rules Fu-3.3.1 to Fu-3.3.4}

The lack of proper rotation axes $C_{\mathrm{n}}(\mathrm{n}>1)$ in these fullerenes requires an approach to helical pathway identification that is different from that employed for fullerenes having axial symmetry. Conceptually, however, the new approach should follow the previous one as much as possible. Accordingly, the following rules are adopted for fullerenes belonging to the $C_{\mathrm{s}}$ point group.

Rule Fu-3.3.1: Since there is no proper rotation axis, the plane of symmetry is used as the reference element. A search is made for a contiguous numbering helix that starts in a ring bisected by the plane of symmetry or along a bond lying in the plane.

Rule Fu-3.3.2: If there is a choice for the beginning of the numbering, rings bisected by the plane are preferred over bonds lying in the plane.

Rule Fu-3.3.2.1: If there is a choice among rings, a larger ring is preferred to a smaller one, and, among rings of the same size, the preferred ring contains the highest-ranking atom at the first point of difference.

Rule Fu-3.3.2.2: If there is a choice among bonds lying in the plane, the preferred bond contains the higher number of higher-ranking atoms. should

Rule Fu-3.3.3: If there is still a choice among contiguous numberings, the preferred pathway 
a. begin, and

b. terminate as close as possible to the reference plane in terms of number of bonds.

Rule Fu-3.3.4: If there is still a choice among contiguous numberings, they are evaluated by sequential comparison of their atom rankings. The preferred pathway contains the highest-ranking atom at the first point of difference.

It must be noted that in the approach used by CAS [3], the plane of symmetry is used as a pivot plane. The spheroidal structure is then sliced into planes parallel to the pivot plane, constructed using specific rules. Either one of the two outer planes can be the reference plane. It should also be mentioned that the assignment of atoms to a given plane in the CAS procedure may depend on the geometry of the structure rather than the connectivity of atoms and can lead to ambiguities.

As a consequence, the numberings produced by CAS are always different from those described here. The following examples illustrate the use of the rules as recommended in this document.

3.3.1 Systematic numbering for $\left(C_{54}-C_{S}\right)[5,6]$ fullerene (Fig. 26) (CAS Reg. No. 136201-95-1) In this fullerene, the plane of symmetry, orthogonal to the plane of the paper in Fig. 26, bisects five sixmembered rings and two five-membered rings. The six-membered rings are preferred for beginning the numbering (rule Fu-3.3.2.1); they are indicated as A, B, C, D, and E in Fig. 26a (smaller-font letters denote rings away from the viewer; larger-font, bold letters denote rings closer to the viewer). Ring A contains only 6,6,5 atoms; rings $\mathrm{B}, \mathrm{C}$, and D contain two 6,6,6 and four 6,6,5 atoms, each; ring E contains four 6,6,6 and two 6,6,5 atoms. Therefore, ring $\mathrm{E}$ is preferred (rule Fu-3.3.2.1). The six atoms of ring $\mathrm{E}$ form three pairs of symmetry-equivalent atoms indicated as $\mathbf{a} / \mathbf{a}, \mathbf{b} / \mathbf{b}^{\prime}$, and $\mathbf{c} / \mathbf{c}^{\prime}$ in Fig. $26 \mathbf{b}$. Because of symmetry, only the six counterclockwise (or clockwise) pathways from each atom must be considered. They all lead to contiguous numbering. However, those starting from atoms $\mathbf{b}, \mathbf{b}^{\prime}, \mathbf{c}$, and $\mathbf{c}^{\prime}$ are preferred because they begin closer $(0.5$ bond) to the reference plane (rule Fu-3.3.3a). Two of these numberings (Figs. 26c and 26d) are preferred because they terminate at an atom 1.5 bonds removed from the reference plane (indicated by a bold dot), whereas the other terminates at an atom 2.5 bonds away from the plane (rule Fu-3.3.3b). Inspection of the atom-ranking in the two preferred sequences reveals that the numbering in Fig. 26c is the correct numbering pathway because it begins with a 6,6,6 atom, whereas that in Fig. 26d begins with a 6,6,5 atom. 


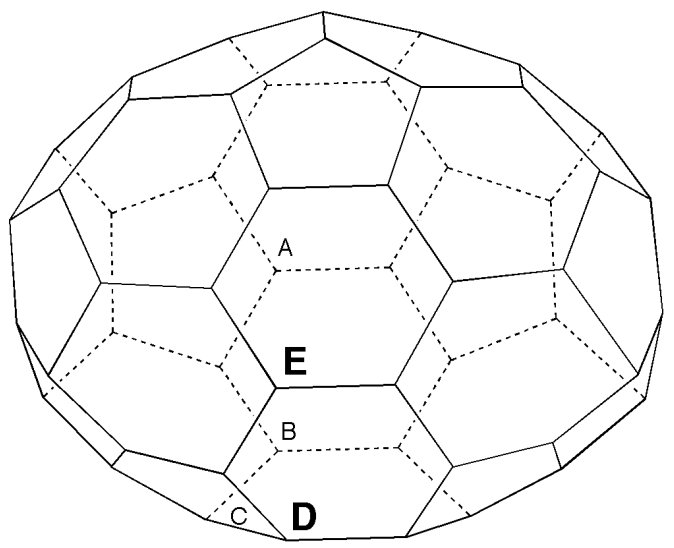

Fig. 26a

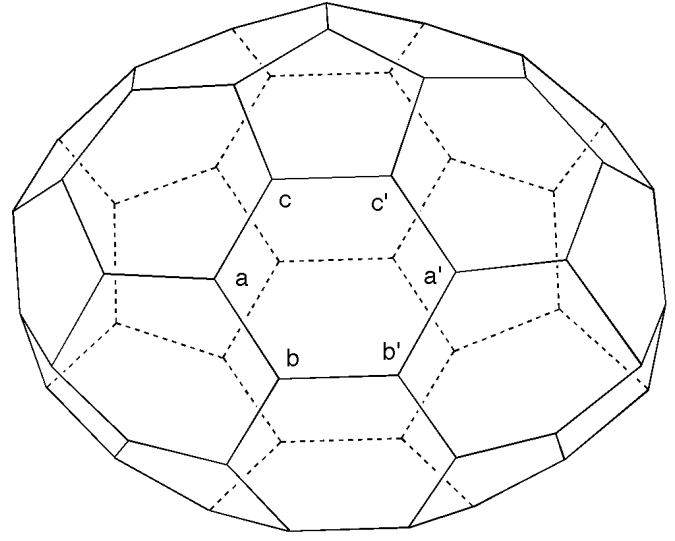

Fig. 26b

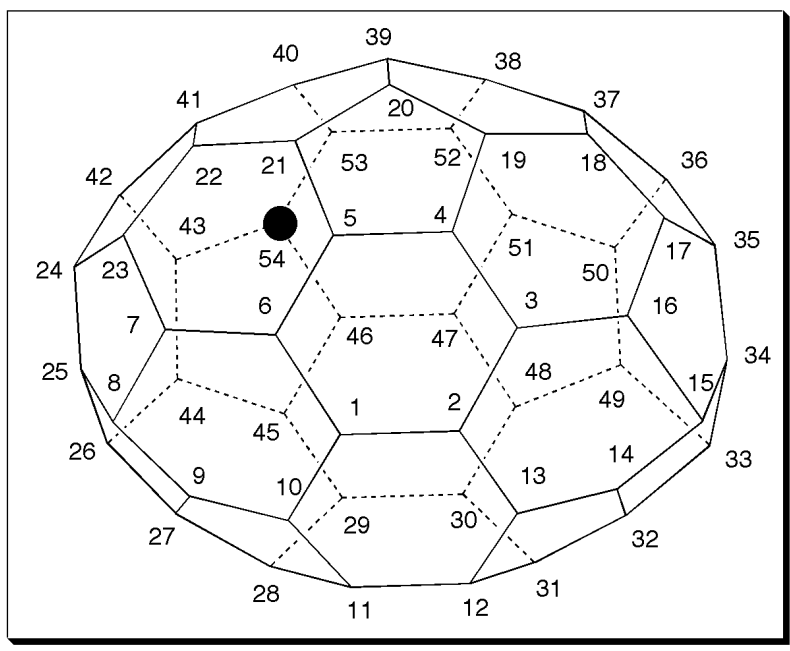

Fig. 26c

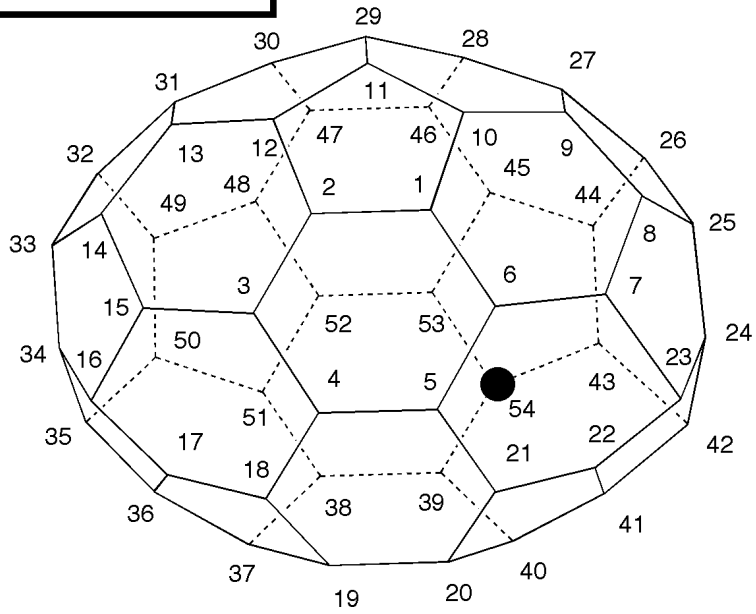

Fig. 26d

Fig. 26 Systematic numbering for $\left(\mathrm{C}_{54^{-}} C_{\mathrm{S}}\right)[5,6]$ fullerene. 


\subsubsection{Systematic numbering for $\left(C_{36}{ }^{-} C_{s}\right)[5,6]$ fullerene (Fig. 27) (Atlas Ref. No. 36:4; CAS Reg. No. 165552-43-2)}

In this fullerene, the plane of symmetry corresponds to the plane of the paper in Fig. 27 and bisects only two six-membered rings which are preferred according to rule Fu-3.3.2.1 indicated as A and B in Fig. 27a. Ring A contains the highest-ranking atom at the first point of difference (namely, four 6,6,5 atoms in a row), whereas ring $B$ contains two pairs of 6,6,5 atoms intercalated by single 6,5,5 atoms. According to rule Fu-3.3.2.1, ring A is preferred for beginning the numbering. There are six pathways to be considered: clockwise and counterclockwise from $\mathbf{a}$, from $\mathbf{b}$, and from $\mathbf{c}$ (Fig. 27b). Of these, only the two starting from $\mathbf{c}$ lead to contiguous numberings, shown in Figs. 27c and 27d, respectively. Since both start from an atom 0.5 bond removed from the plane of symmetry and terminate at an atom lying on the plane of symmetry, they cannot be distinguished on the basis of rule Fu-3.3.3. However, application of rule Fu-3.3.4 shows that the numbering reported in Fig. 27d must be preferred because it features a 6,6,5 atom at position 2 instead of the 6,5,5 atom of the numbering of Fig. 27c.

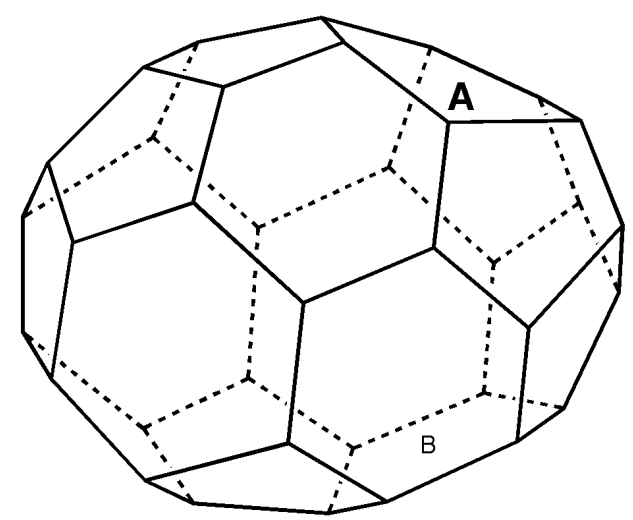

Fig. 27a

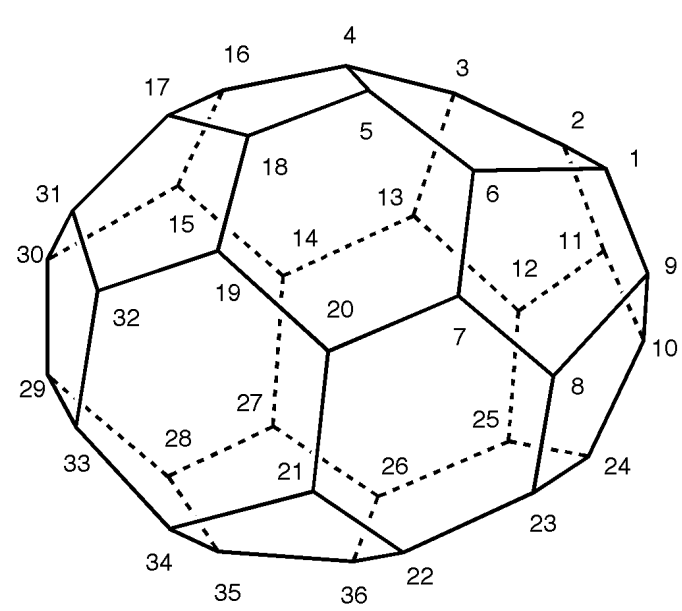

Fig. 27c

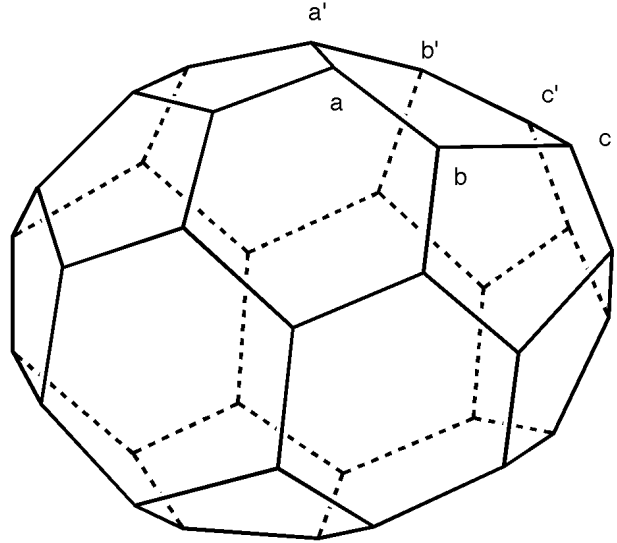

Fig. 27b

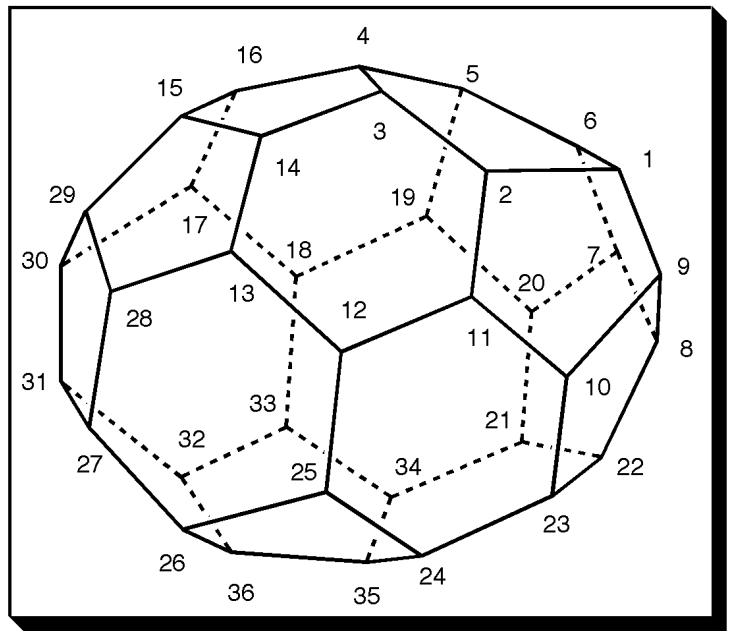

Fig. 27d

Fig. 27 Systematic numbering for $\left(\mathrm{C}_{36^{-}} C_{\mathrm{s}}\right)[5,6]$ fullerene. 


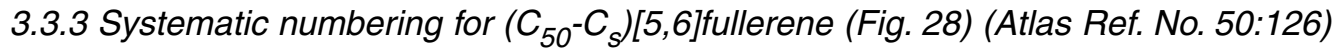

In this fullerene, the plane of symmetry bisects three six-membered rings indicated as $\mathrm{A}, \mathrm{B}$, and $\mathrm{C}$ in Fig. 28a. Ring A contains a highest-ranking atom at the first point of difference than both rings $\mathrm{B}$ or $\mathrm{C}$ (ring A: four contiguous 6,6,5 atoms; rings B and C: two pairs of 6,6,5 atoms intercalated by single 6,5,5 atoms), and is therefore preferred to begin the numbering (rule Fu-3.3.2.1). However, none of the six possible pathways from A (clockwise and counterclockwise from $\mathbf{a}, \mathbf{b}$, and $\mathbf{c}$ ) is contiguous. There are six pathways to be examined from ring B: from $\mathbf{d}$ and $\mathbf{g}$ (because these atoms are lying in the plane of symmetry, it does not matter whether the numbering proceeds clockwise or counterclockwise), and clockwise and counterclockwise from $\mathbf{e}$ and $\mathbf{f}$; and six from ring $\mathrm{C}$ : from $\mathbf{h}$ and $\mathbf{k}$ (because these atoms are lying in the plane of symmetry, it does not matter whether the numbering proceeds clockwise or counterclockwise), and clockwise and counterclockwise from $\mathbf{i}$ and $\mathbf{j}$. Of these, only those from $\mathbf{d}, \mathbf{g}$, and the clockwise numbering from $\mathbf{j}$ are contiguous. They are shown in Figs. 28b, 28c, and 28d, respectively. Among these numberings, the one from $\mathbf{j}$ (Fig. 28d) is discarded because it begins out of the plane, whereas the others begin in the plane (rule Fu-3.3.3a). Application of rule Fu-3.3.3b does not help, since both numberings terminate at an atom 0.5 bond away from the plane. Eventually, a selection is made in favor of the numbering reported in Fig. 28c, because it features a 6,6,5 atom instead of a 6,5,5 atom at position 8 (Fu-3.3.4). 


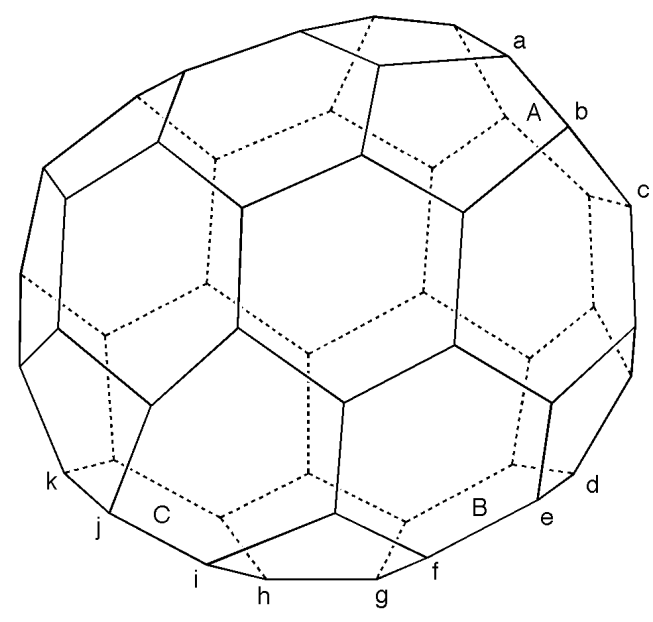

Fig. 28a

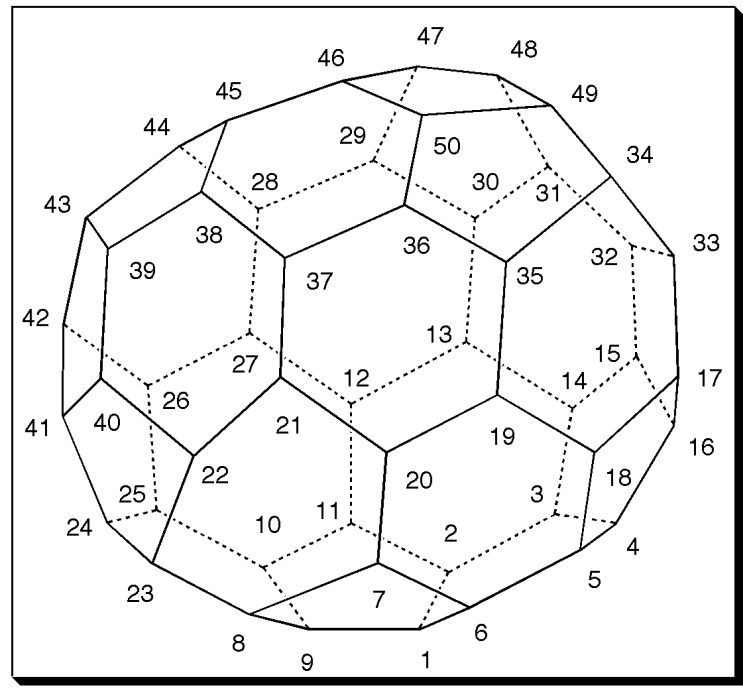

Fig. 28c

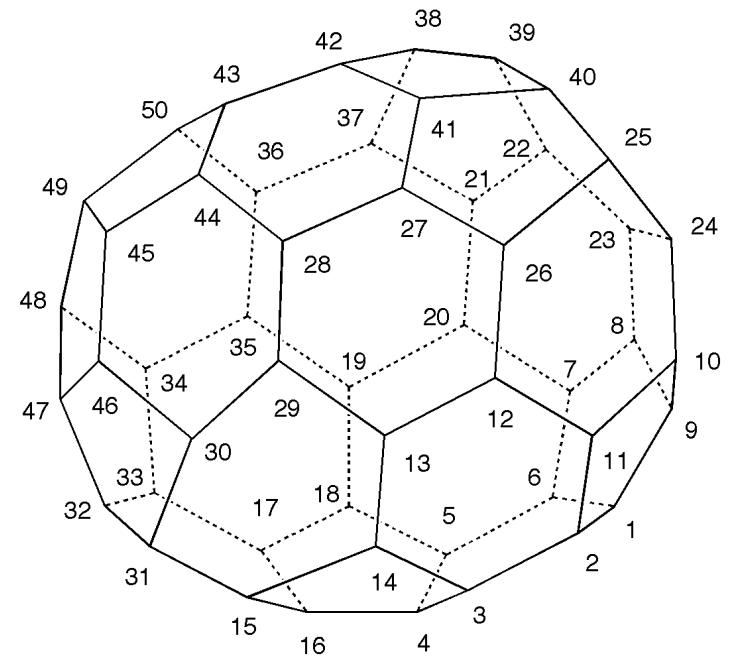

Fig. 28b

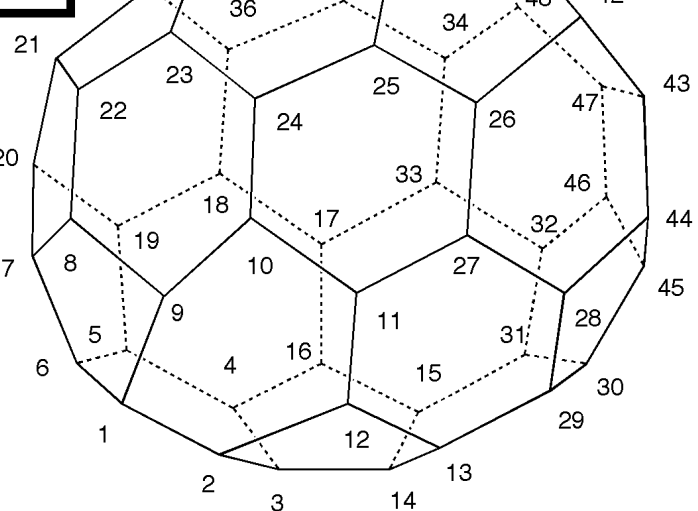

Fig. 28d

Fig. 28 Systematic numbering for $\left(\mathrm{C}_{50}-C_{\mathrm{s}}\right)[5,6]$ fullerene. 


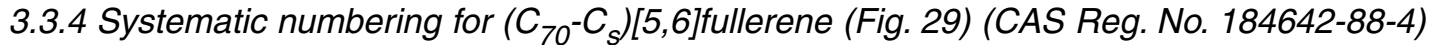
In this fullerene, the plane of symmetry bisects three six-membered rings indicated as $\mathrm{A}, \mathrm{B}$, and $\mathrm{C}$ in Fig. 29a. Ring A contains two 6,6,6 atoms, and is therefore preferred to begin the numbering over the
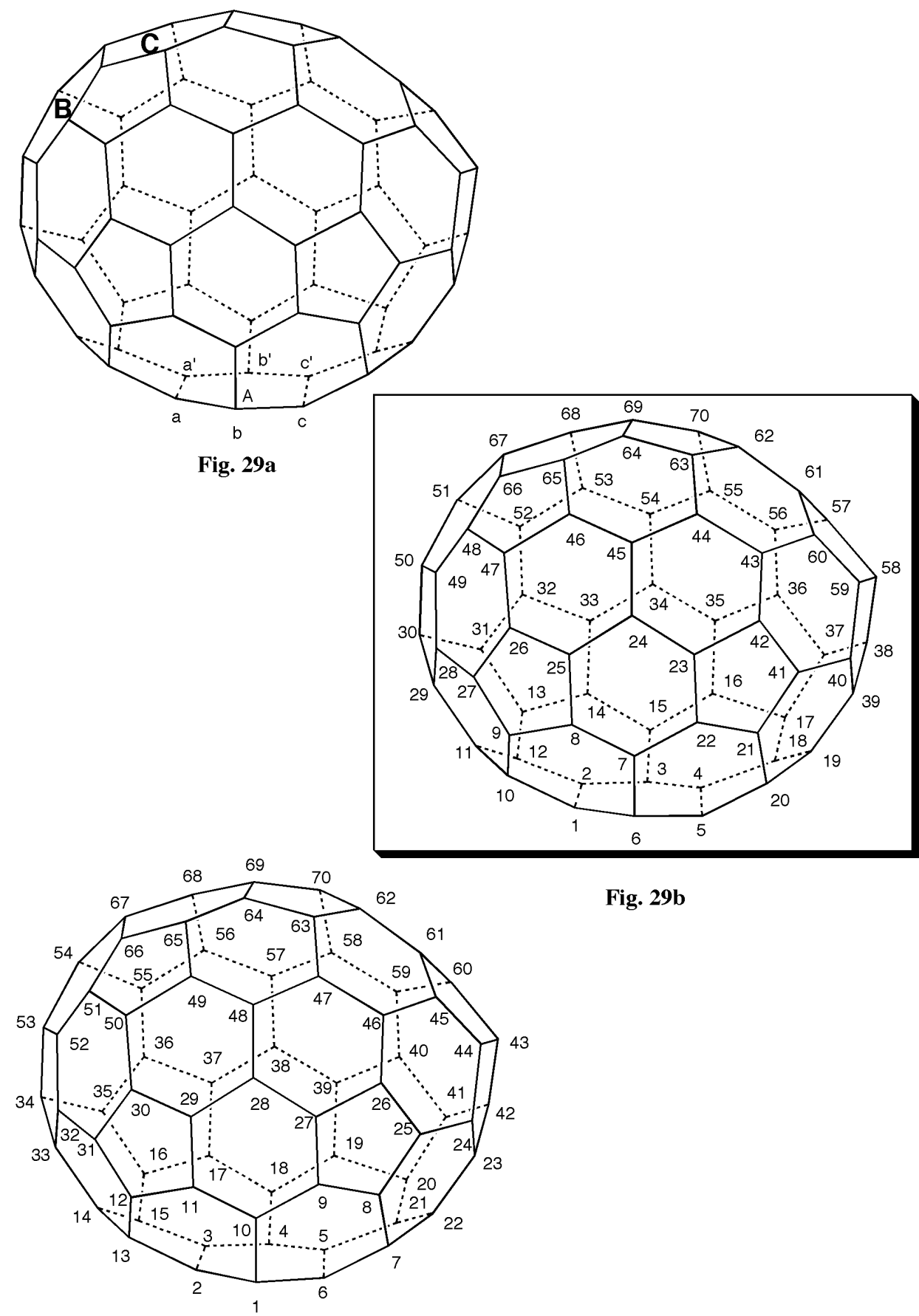

Fig. 29b

Fig. 29c

Fig. 29 Systematic numbering for $\left(\mathrm{C}_{70}-C_{\mathrm{s}}\right)[5,6]$ fullerene. 
other six-membered rings that contain only 6,6,5 atoms (rule Fu-3.3.2.1). Of the six pathways to be considered from ring A (clockwise and counterclockwise from $\mathbf{a}, \mathbf{b}$, and $\mathbf{c}$ ), only those proceeding counterclockwise from $\mathbf{a}$ and counterclockwise from $\mathbf{b}$ lead to contiguous numberings. The former, shown in Fig. 29b, begins at an atom 0.5 bond away from the reference plane; the latter (Fig. 29c) begins at an atom 1.5 bonds away from the plane. According to Fu-3.3.3a, the former (Fig. 29b) is correct.

\subsubsection{Systematic numbering for $\left(C_{82}-C_{s}\right)[5,6]$ fullerene (Fig. 30) (Atlas Ref. No. 82:2; CAS Reg. No. 145954-07-0)}

In this fullerene, the plane of symmetry bisects five six-membered rings indicated as A, B, C, D, and E in Fig. 30a. Ring C contains only 6,6,5 atoms; rings $B$ and $E$ contain two 6,6,6 and four 6,6,5 atoms, each; ring A contains four 6,6,6 and two 6,6,5 atoms; ring D contains only 6,6,6 atoms, and thus is preferred to begin the numbering (rule Fu-3.3.2.1). However, none of the pathways from this ring is contiguous, and the pathways from ring A, which contains a sequence of four 6,6,6 atoms, must be examined. The atoms of this ring are indicated as $\mathbf{a} / \mathbf{a}^{\prime}, \mathbf{b} / \mathbf{b}^{\prime}$, and $\mathbf{c} / \mathbf{c}^{\prime}$ in Fig. 30a. There are six clockwise pathways to be examined, one from each atom. Only those from a (Fig. 30b) and from b' (Fig. 30c) are contiguous. The latter is preferred because it starts closer to the reference plane $(\mathbf{F u}-\mathbf{3 . 3 . 3 a})$. 


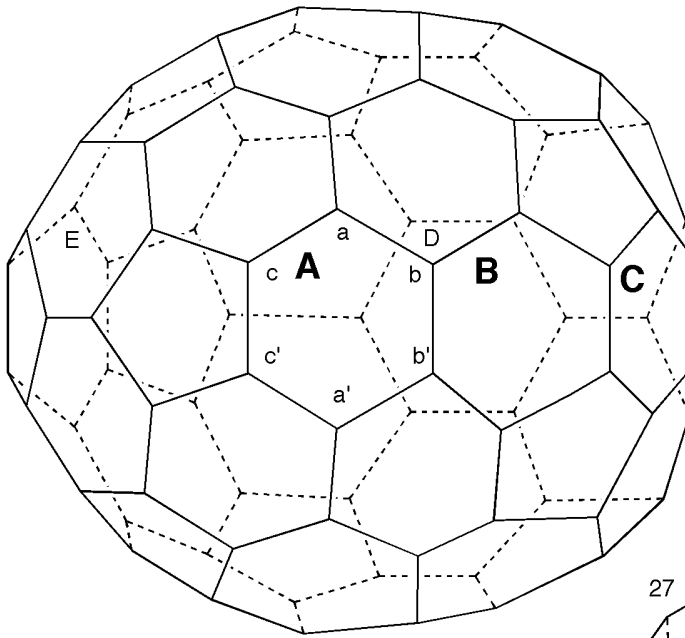

Fig. 30a
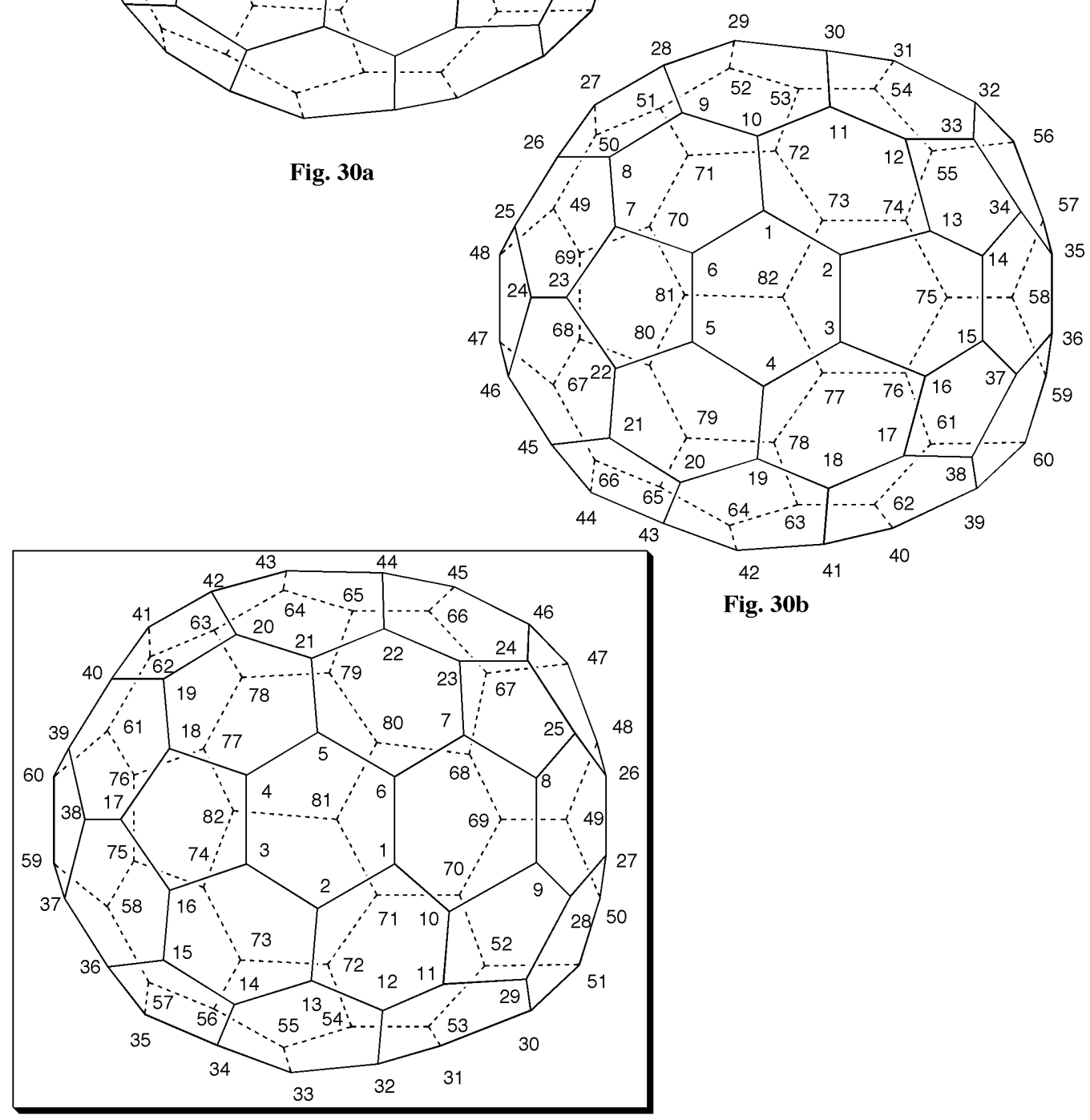


\subsection{Fullerenes belonging to the $C_{\mathrm{i}}$ or $C_{1}$ point groups and having a contiguous helical numbering pathway determined unambiguously}

In the achiral $C_{\mathrm{i}}$ fullerenes, the center of inversion $i$ is the only symmetry element. Different from the fullerenes examined so far, there are neither axes nor planes of symmetry passing through or lying upon a ring, a bond, or an atom, and the symmetry element, being inside the spheroid, cannot be used as a reference to begin the numbering. The chiral $C_{1}$ fullerenes can be made identical to themselves only by a $360^{\circ}$ rotation around any axis passing anywhere through the fullerene. Therefore, no axis can be used as a reference to begin the numbering since it does not have an unambiguously determined location.

For both $C_{\mathrm{i}}$ and $C_{1}$ fullerenes, the absence of appropriate reference elements could lead to an undesirable increase of the number of possible numbering pathways. Therefore, the primary requirement of any numbering system should be the reduction of the allowed numbering options. Much in line with the procedure employed for the numbering of other fullerenes and in particular of $C_{\mathrm{s}}$ fullerenes, the following rules are adopted in order to decide where to begin the numbering.

Rule Fu-3.4.1: A larger ring is preferred to a smaller one to begin the numbering; among rings of the same size, the preferred ring contains the highest-ranking atom at the first point of difference.

Rule Fu-3.4.2: If there is still a choice among contiguous numberings, they are compared according to their atom-ranking sequence. The preferred pathway contains the highest-ranking atom at the first point of difference.

The numbering of $C_{\mathrm{i}}$ and $C_{1}$ fullerenes proposed by CAS [3] is also based on the identification of a preferred ring to begin the numbering. Incidentally, no example of numbering of $C_{\mathrm{i}}$ fullerenes has been reported by CAS.

\subsubsection{Systematic numbering for $\left(C_{56}-C_{i}\right)[5,6] f u l l e r e n e$ (Fig. 31) (see ref. [5], p. 100)}

This fullerene contains nine pairs of symmetry-equivalent six-membered rings preferred for the beginning of the numbering according to rule Fu-3.4.1. They are related by the center of inversion (shown by a bold dot in Fig. 31a). Symmetry-equivalent rings are identified by the same letter, unprimed and primed (in Fig. 31a, primed letters indicate rings away from the viewer and bold letters rings closer to the viewer). Because of symmetry, only one set of nine nonequivalent six-membered rings is considered (those denoted by unprimed letters). Each of these rings is eligible to begin the numbering, and they are ranked on the basis of their atoms (rule Fu-3.4.1). Rings B, C, and D contain four contiguous 6,6,6 atoms and two 6,6,5 atoms, and are preferred for beginning the numbering. Please note that some of the other rings also contain four 6,6,6 and two 6,6,5 atoms, but the 6,6,6 atoms are not contiguous. Since the pathways having four contiguous $6,6,6$ atoms at position 1-4 would be preferred according to rule $\mathbf{F u - 3 . 4 . 2}$, they are examined first for contiguous numbering to reduce the number of necessary inspections. The 6,6,6 atoms are indicated by the letters $\mathbf{a}$ to $\mathbf{g}$ in Fig. $31 \mathrm{~b}$. There are six pathways to be considered: $\mathbf{a}$ to $\mathbf{b}$ to $\mathbf{c}$ to $\mathbf{d}$ and $\mathbf{d}$ to $\mathbf{c}$ to $\mathbf{b}$ to $\mathbf{a}$, from ring $\mathbf{C} ; \mathbf{b}$ to $\mathbf{c}$ to $\mathbf{f}$ to $\mathbf{g}$ and $\mathbf{g}$ to $\mathbf{f}$ to $\mathbf{c}$ to $\mathbf{b}$, from ring $B ; \mathbf{f}$ to $\mathbf{c}$ to $\mathbf{d}$ to $\mathbf{e}$ and $\mathbf{e}$ to $\mathbf{d}$ to $\mathbf{c}$ to $\mathbf{f}$, from ring D. Only one of these pathways ( $\mathbf{d}$ to $\mathbf{c}$ to $\mathbf{b}$ to $\mathbf{a}$, from ring $\mathrm{C}$ ) leads to a contiguous numbering, that is shown in Fig. 31c. 

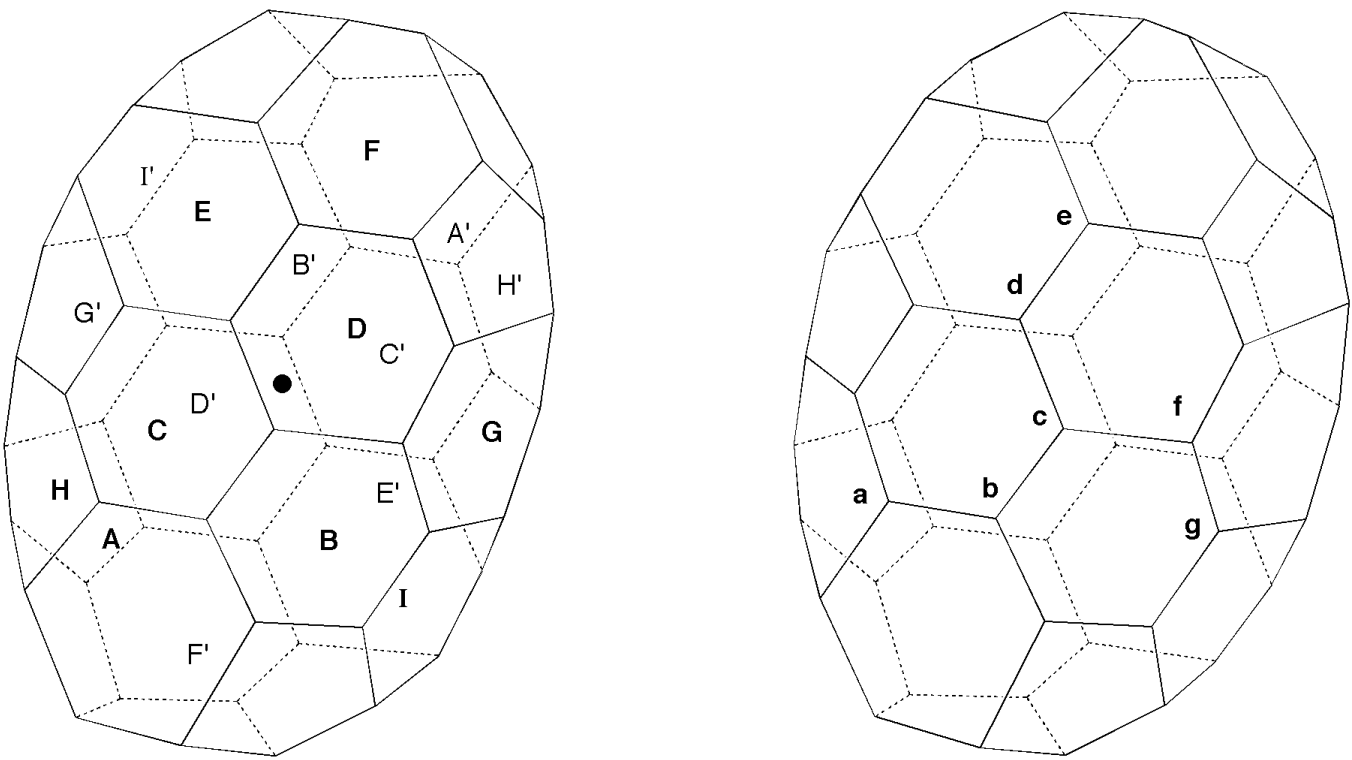

$31 \mathbf{a}$

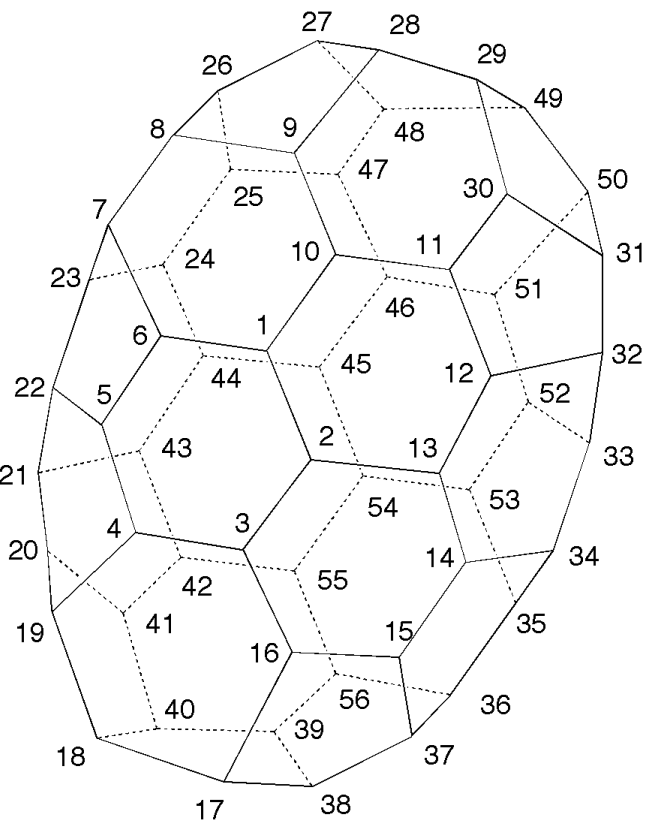

$31 b$

$31 \mathrm{c}$

Fig. 31 Systematic numbering for $\left(\mathrm{C}_{56^{-}} C_{\mathrm{i}}\right)[5,6]$ fullerene.

\subsubsection{Systematic numbering for $\left(C_{36}-C_{1}\right)[5,6]$ fullerene (Fig. 32) (Atlas Ref. No. 36:7;} CAS Reg. No. 165552-38-5)

This fullerene contains eight six-membered rings indicated by the letters A to $\mathrm{H}$ in Fig. 32a (smallerfont letters denote rings away from the viewer; larger-font, bold letters denote rings closer to the viewer). According to rule Fu-3.4.1, ring $\mathrm{H}$ is preferred to begin the numbering since it contains the highest-ranking atom at the first point of difference. There are 12 pathways to be considered in ring $\mathrm{H}$, 


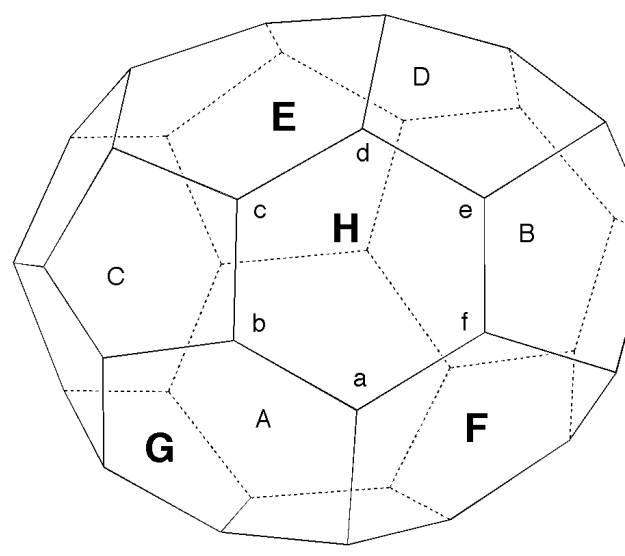

Fig. 32a
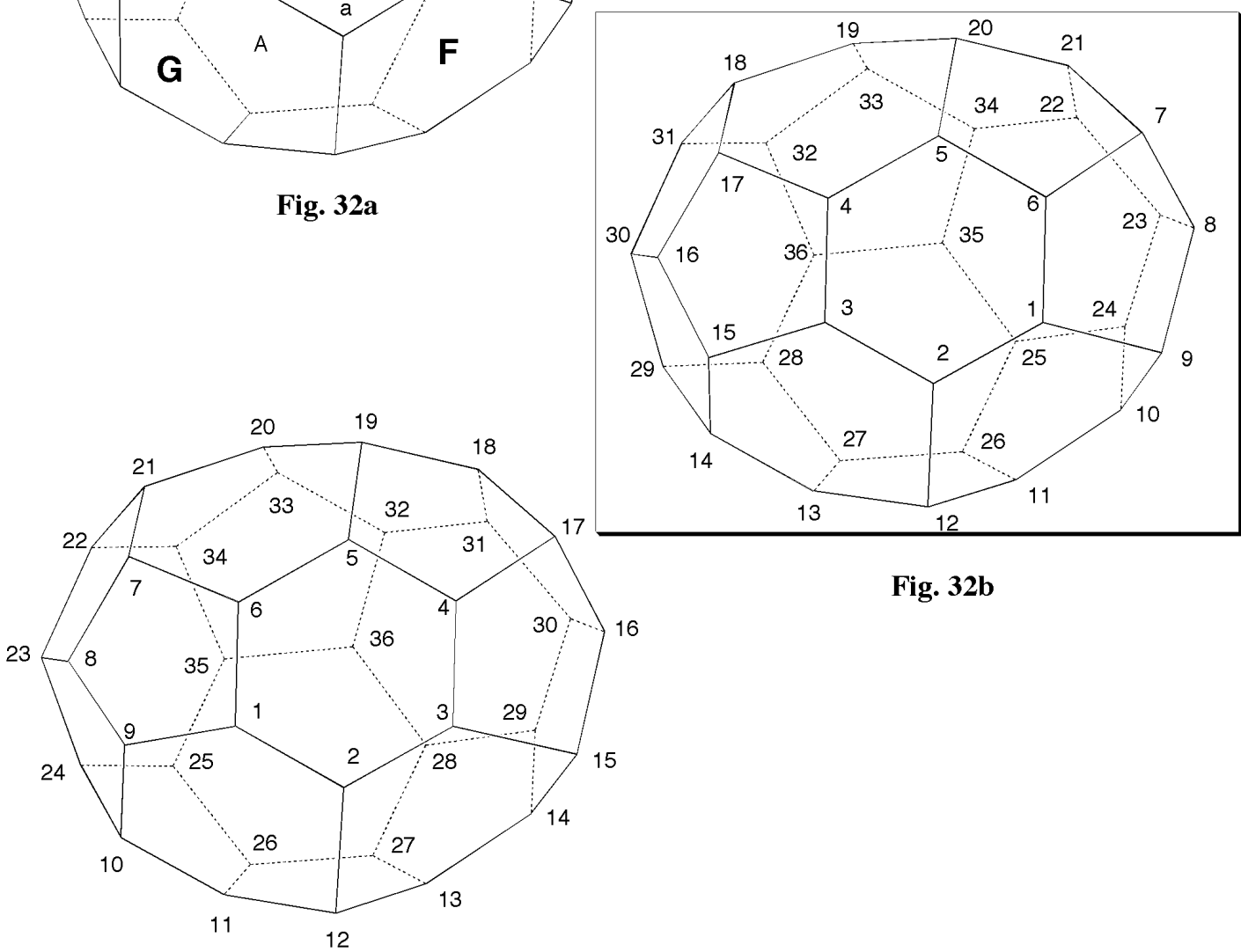

Fig. 32b

Fig. 32c

Fig. 32 Systematic numbering for $\left({ }^{\mathrm{f}, \mathrm{s}} C\right)\left(\mathrm{C}_{36^{-}} C_{1}\right)[5,6]$ fullerene.

clockwise and counterclockwise from each atom a-f. In order to reduce the number of pathways to be actually examined, those beginning from the 6,6,6 atom (clockwise and counterclockwise) are studied first, because, if contiguous, these pathways will be preferred to any other pathway starting in this ring according to rule $\mathbf{F u - 3 . 4 . 2}$. Unfortunately, they are both discontiguous. The two pathways having the 6,6,6 atom a at position 2 are then examined for contiguous numbering: again, these would be preferred at this point, since no other pathway can have a 6,6,6 atom at position 2. The two pathways are clockwise from atom $\mathbf{f}$, and counterclockwise from atom $\mathbf{b}$. They lead to the contiguous numberings shown in Figs. 32b and 32c, respectively. These contiguous sequences are then compared using rule Fu-3.4.2 to discover that there is a 6,6,5 atom at position 4 in the pathway from atom $\mathbf{f}$ (Fig. 32b) and a 6,5,5 atom at the same position in the pathway from atom $\mathbf{b}$ (Fig. 32c). Therefore, the numbering sequence shown in Fig. 32b, is correct. The enantiomer shown here has the $\left({ }^{\mathrm{f}, \mathrm{s}} \mathrm{C}\right)$ configuration [4]. 


\subsubsection{Systematic numbering for $\left(C_{44}-C_{1}\right)[5,6]$ fullerene (Fig. 33) (Atlas Ref. No. 44:10; CAS} Reg. No. 573999-42-5)

This fullerene contains twelve six-membered rings shown as A to L in Fig. 33a (smaller-font letters denote rings away from the viewer; larger-font, bold letters denote rings closer to the viewer). Among these, rings $\mathrm{C}$, I, and $\mathrm{K}$ are preferred according to rule $\mathbf{F u - 3 . 4 . 1}$ to begin the numbering, since they contain the higher-ranking atoms at the first point of difference. From these rings, there are in principle thirty-six pathways to be examined. To reduce the number of pathways to be actually examined, the six pathways featuring the three 6,6,6 atoms at the first three positions are examined first, since, if contiguous, they will be preferred to all other possible pathways according to rule $\mathbf{F u - 3 . 4 . 2}$. Only the two pathways beginning in ring I (clockwise from atom a, and counterclockwise from atom $\mathbf{c}$ in Fig. 33b, which shows a different view of $\left(\mathrm{C}_{44^{-}} C_{1}\right)[5,6]$ fullerene) lead to contiguous spiral numberings. These are shown in Figs. 33c and 33d. These numbering sequences are compared using Fu-3.4.2 to discover that there is a 6,6,5 atom at position 23 in the sequence of Fig. $33 \mathrm{c}$ and a 6,5,5 atom at the same position in the sequence of Fig. 33d. The former is, therefore, preferred. The configuration of the shown enantiomer is $\left({ }^{\mathrm{f}, \mathrm{s}} A\right)[4]$.

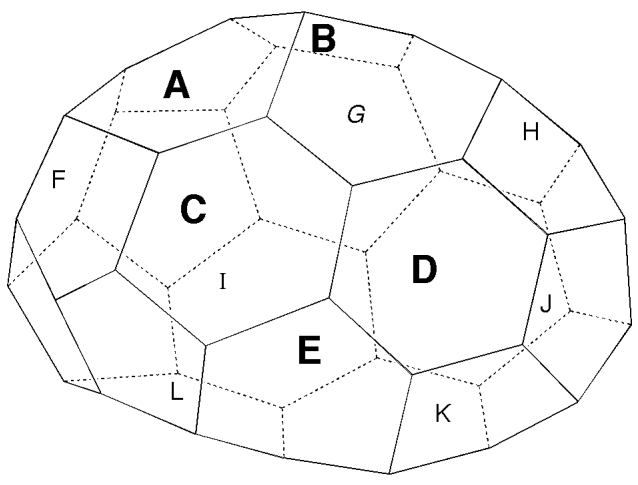

Fig. 33a

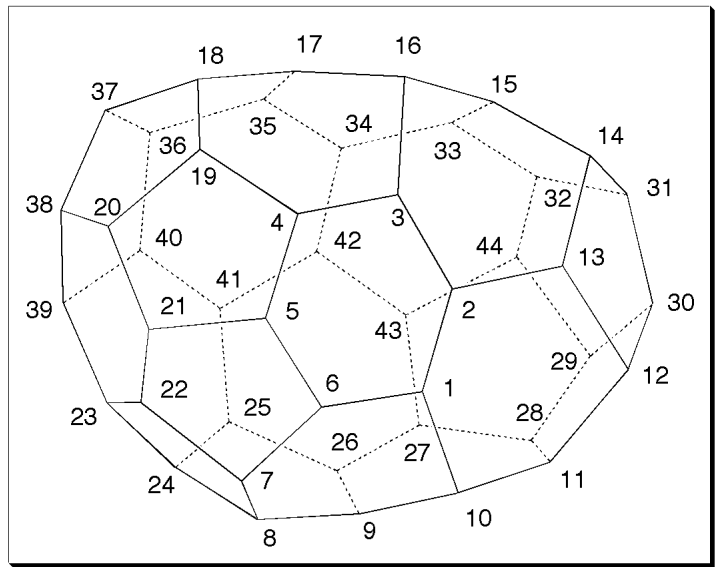

Fig. 33c

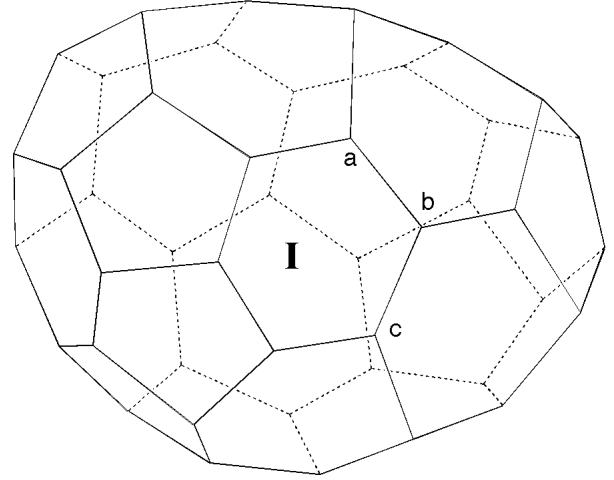

Fig. 33b

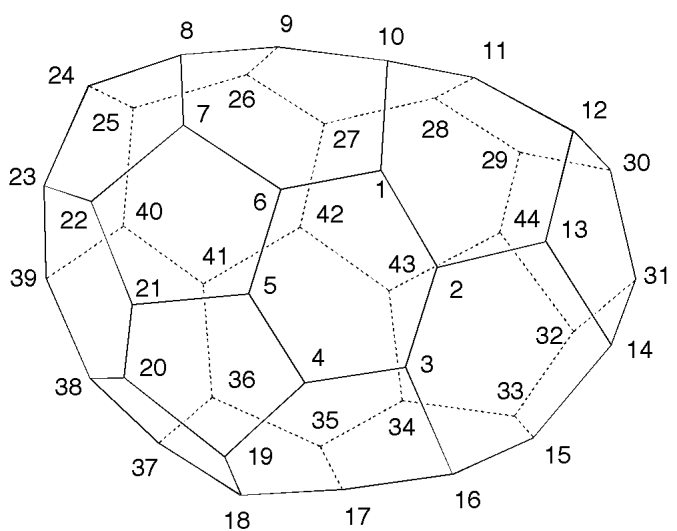

Fig. 33d

Fig. 33 Systematic numbering for $\left({ }^{\mathrm{f}, \mathrm{s}} A\right)\left(\mathrm{C}_{44^{-}} C_{1}\right)[5,6]$ fullerene. 
3.4.4 Systematic numbering for $\left(C_{94}-C_{1}\right)[5,6]$ fullerene (Fig. 34) (Atlas Ref. No. 94:12; CAS Reg. No. 401792-31-2)

This fullerene contains only $6,6,6$ or $6,6,5$ atoms and thirty-six six-membered rings. Seventeen rings contain a sequence of four 6,6,6 and two 6,6,5 atoms, and the numbering should begin from these rings according to Fu-3.4.1. Of all the possible numberings, the seventeen clockwise and the seventeen counterclockwise pathways featuring the four 6,6,6 atoms in a row at the beginning of the numbering are considered first, since, if contiguous, they will be preferred according to rule Fu-3.4.2. Eleven of these pathways lead to contiguous numberings: clockwise from rings A, B, C, E, and F; counterclockwise from rings D, E, G, H, I, and J (Fig. 34a; smaller-font letters denote rings away from the viewer; larger-font, bold letters denote rings closer to the viewer). Comparison of the atom sequences by application of Fu-3.4.2 eliminates five numberings because of the ranking of the atoms at position 8, two of the six remaining numberings at position 9 , and two of the four remaining numberings at position 11 . This leaves only the two numberings reported in Figs. 34b (clockwise from ring C) and 34c (counterclockwise from ring J). Selection between these can be made by inspection of the atoms up to position 15, which is a 6,6,6 atom in the numbering of Fig. $34 \mathrm{~b}$ and a 6,6,5 atom in the numbering of Fig. 34c. The former is, therefore, preferred based on rule $\mathbf{F u - 3 . 4 . 2}$. The configuration of the shown enantiomer is $\left({ }^{\mathrm{f}, \mathrm{s}} C\right)[4]$.

Finally, it must be noted that this document does not deal with $C_{\mathrm{s}}, C_{\mathrm{i}}$, and $C_{1}$ fullerenes without contiguous numbering, of which no examples were encountered during the elaboration of the present document. Since these fullerenes have a large number of possible numbering pathways, it seems rather unlikely that a contiguous numbering cannot be found for most of these structures. In any case, application of rules Fu-3.2.1.a, Fu-3.2.3, and Fu-3.2.4 should allow the user to identify a preferred discontiguous numbering. 

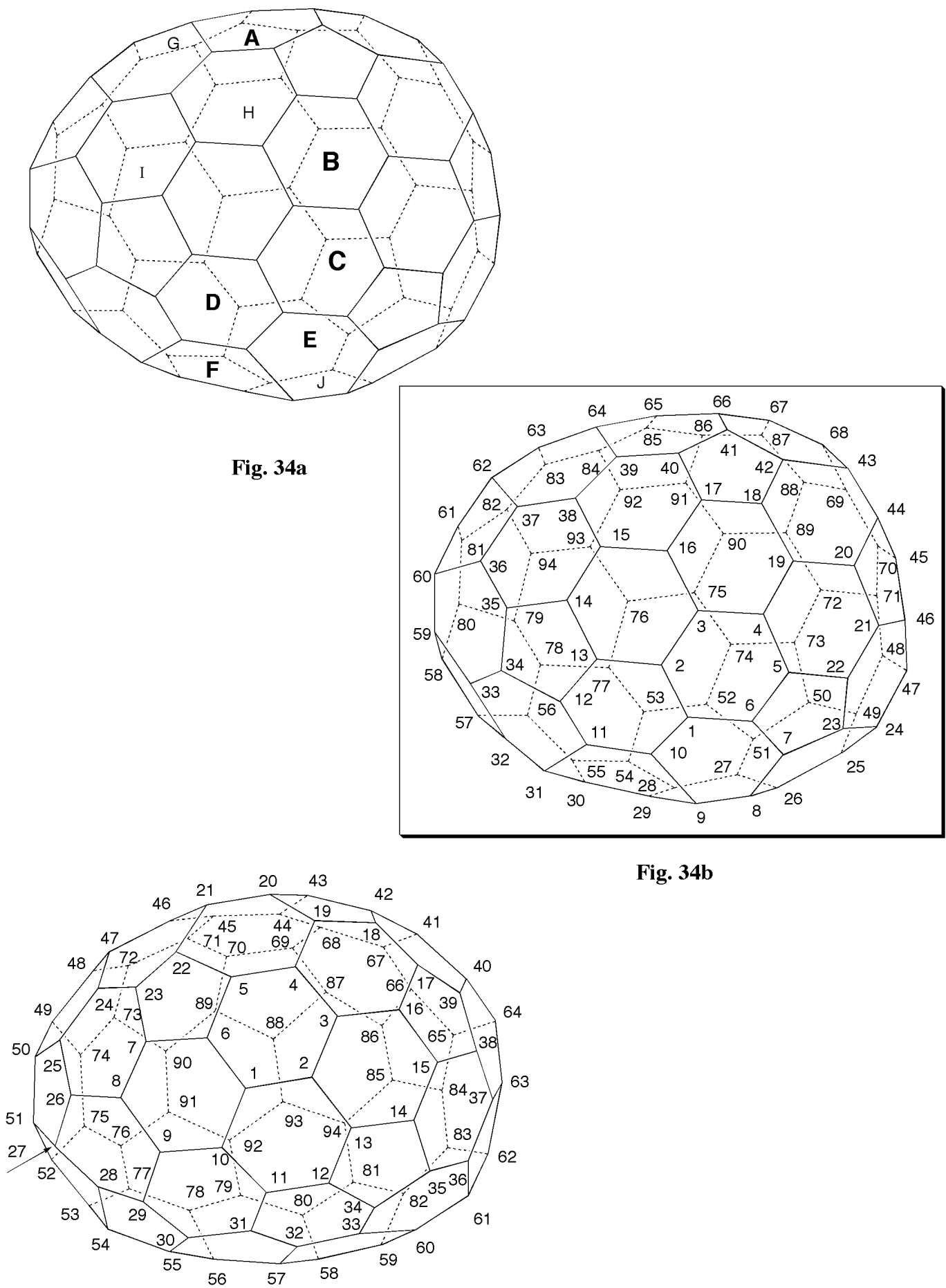

Fig. 34b

Fig. 34c

Fig. 34 Systematic numbering for $\left({ }^{\mathrm{f}, \mathrm{s}} C\right)\left(\mathrm{C}_{94}{ }^{-} C_{1}\right)[5,6]$ fullerene. 


\section{REFERENCES}

1. International Union of Pure and Applied Chemistry. "Nomenclature and terminology of fullerenes: A preliminary report", prepared by E. W. Godly and R. Taylor, Pure Appl. Chem. 69, 1411-1434 (1997).

2. R. Taylor. J. Chem. Soc., Perkin Trans. 2, 813-824 (1993).

3. A. L. Goodson, C. L. Gladys, D. E. Worst. J. Chem. Inf. Comp. Sci. 35, 969-978 (1995).

4. International Union of Pure and Applied Chemistry, Division of Organic Chemistry, Commission on Nomenclature of Organic Chemistry. "Nomenclature for the $\mathrm{C}_{60}-I_{\mathrm{h}}$ and $\mathrm{C}_{70}-D_{5 \mathrm{~h}(6)}$ fullerenes (IUPAC Recommendations 2002)", Pure Appl. Chem. 74, 629-695 (2002).

5. P. W. Fowler and D. E. Manolopoulos. An Atlas of Fullerenes, Clarendon Press, Oxford (1995). 


\title{
APPENDIX 1: RULES
}

\section{Fullerenes having at least one symmetry axis $\left(C_{n}, n>1\right)$ and a contiguous helical pathway}

Fullerenes of this type without a contiguous helical pathway determined on the basis of rules Fu-3.1.1 to $\mathbf{F u - 3 . 1 . 4}$ are dealt with in rules $\mathbf{F u - 3 . 2 . 1}$ to $\mathbf{F u - 3 . 2 . 4}$.

Rule Fu-3.1.1: Proper rotation axes $\left(C_{n}\right)$ are examined in sequence from the highest-order to the lowest-order axis, until at least one contiguous helical pathway is found that begins in a ring through which a proper rotation axis passes, at one end of a bond bisected by a proper rotation axis, or at an atom through which a proper rotation axis passes. Numbering begins at the end of such a contiguous helical pathway, and the corresponding axis is called the "reference axis".

Rule Fu-3.1.2: If there is a choice for the selection of a reference axis or for the end of the reference axis to begin the numbering, a ring is preferred to a bond which, in turn, is preferred to an atom.

Rule Fu-3.1.2.1: When there is a choice between rings for the beginning of numbering, a larger ring is preferred to a smaller one. When there is still a choice, the preferred ring contains the highestranking atom at the first point of difference. The highest-ranking atom is defined as the common atom of the set of three largest rings. For example, in a [5,6]fullerene, an atom at the intersection of three sixmembered rings (in short, a 6,6,6 atom) ranks higher than an atom at the intersection of two six-membered rings and a five-membered ring (a 6,6,5 atom).

Rule Fu-3.1.2.2: When there is a choice between bonds bisected by an axis for the beginning of numbering, the preferred bond contains the higher number of higher-ranking atoms.

Rule Fu-3.1.2.3: When there is a choice between atoms lying on an axis for the beginning of numbering, the numbering must start at the higher-ranking atom.

Rule Fu-3.1.3: When there is a choice among helical numbering pathways, the preferred pathway terminates as close as possible, in terms of the number of bonds, to the reference axis.

Rule Fu-3.1.4: When there is still a choice among helical pathways for numbering, they are evaluated by sequential comparison of the ranking of their atoms. The preferred pathway contains the highest-ranking atom at the first point of difference.

\section{Fullerenes having at least one symmetry axis $\left(C_{n}, n>1\right)$, but no contiguous helical numbering pathway determined on the basis of rules Fu-3.1.1 to Fu-3.1.4}

\author{
Rule Fu-3.2.1 (cf. rule Fu-3.1.1):
}

a. The $\mathrm{n}^{\text {th }}$ discontiguity must occur as late as possible in the numbering.

b. Among different pathways having the $\mathrm{n}^{\text {th }}$ discontiguity at the same highest locant, the preferred numbering pathway is built around the proper rotation axis of higher order.

Rule Fu-3.2.2 (cf. rule Fu-3.1.2): If there is a choice among reference axes of the same order or between the end of a reference axis to begin the numbering, rule $\mathbf{F u - 3 . 1 . 2}$ is applied.

Rule Fu-3.2.3 (cf. rule Fu-3.1.4; for practical reasons, no rules analogous to rule $\mathbf{F u - 3 . 1 . 3}$ are applied to segments of discontiguous numberings): When there is still a choice, the $\mathrm{n}^{\text {th }}$ segments are compared according to rule $\mathbf{F u - 3 . 1 . 4 . ~}$

Once a preferred segment is identified based on rules Fu-3.2.1 to Fu-3.2.3, the numbering of the fullerene is completed by construction of the subsequent segment(s). The starting point of a new segment is defined according to rule Fu-3.2.4a (see below), and numbering within a segment is continued according to rule $\mathbf{F u - 3 . 2 . 4 b}$.

Rule Fu-3.2.4: After a discontiguity,

a. the next atom to be numbered must be directly connected to one of the previously numbered atoms; among the latter, the atom with the highest locant is preferred. 
b. numbering continues by the shortest path, in terms of number of bonds, to reach an unnumbered atom that is directly connected to a numbered atom with the lowest locant already assigned. (In this way, the sense of the numbering helix should be maintained, and its pitch kept as small as possible.)

\section{Fullerenes belonging to the $C_{\mathrm{s}}$ point group and having a contiguous helical pathway}

Rule Fu-3.3.1: Since there is no proper rotation axis, the plane of symmetry is used as the reference element. A search is made for a contiguous numbering helix that starts in a ring bisected by the plane of symmetry or along a bond lying in the plane.

Rule Fu-3.3.2: If there is a choice for the beginning of the numbering, rings bisected by the plane are preferred over bonds lying in the plane.

Rule Fu-3.3.2.1: If there is a choice among rings, a larger ring is preferred to a smaller one and, among rings of the same size, the preferred ring contains the highest-ranking atom at the first point of difference.

Rule Fu-3.3.2.2: If there is a choice among bonds lying in the plane, the preferred bond contains the higher number of higher-ranking atoms.

Rule Fu-3.3.3: If there is still a choice among contiguous numberings, the preferred pathway should:

a. begin, and

b. terminate as close as possible to the reference plane in terms of number of bonds.

Rule Fu-3.3.4: If there is still a choice among contiguous numberings, they are evaluated by sequential comparison of their atom rankings. The preferred pathway contains the highest-ranking atom at the first point of difference.

\section{Fullerenes belonging to the $C_{\mathrm{i}}$ or $C_{1}$ point groups and having a contiguous helical numbering pathway}

Rule Fu-3.4.1: A larger ring is preferred to a smaller one to begin the numbering; among rings of the same size, the preferred ring contains the highest-ranking atom at the first point of difference.

Rule Fu-3.4.2: If there is still a choice among contiguous numberings, they are compared according to their atom-ranking sequence. The preferred pathway contains the highest-ranking atom at the first point of difference. 


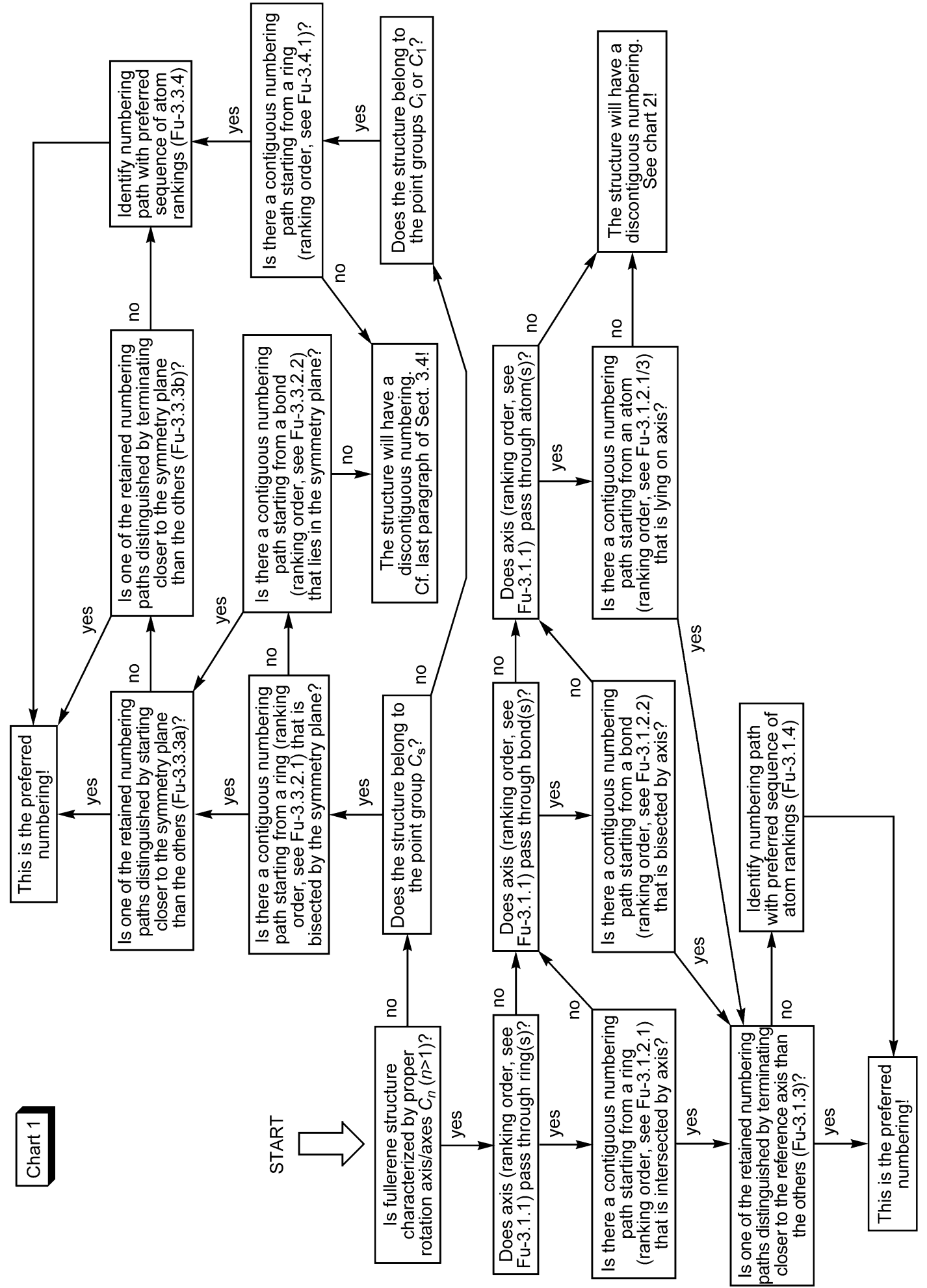

(C) 2005 IUPAC, Pure and Applied Chemistry 77, 843-923 


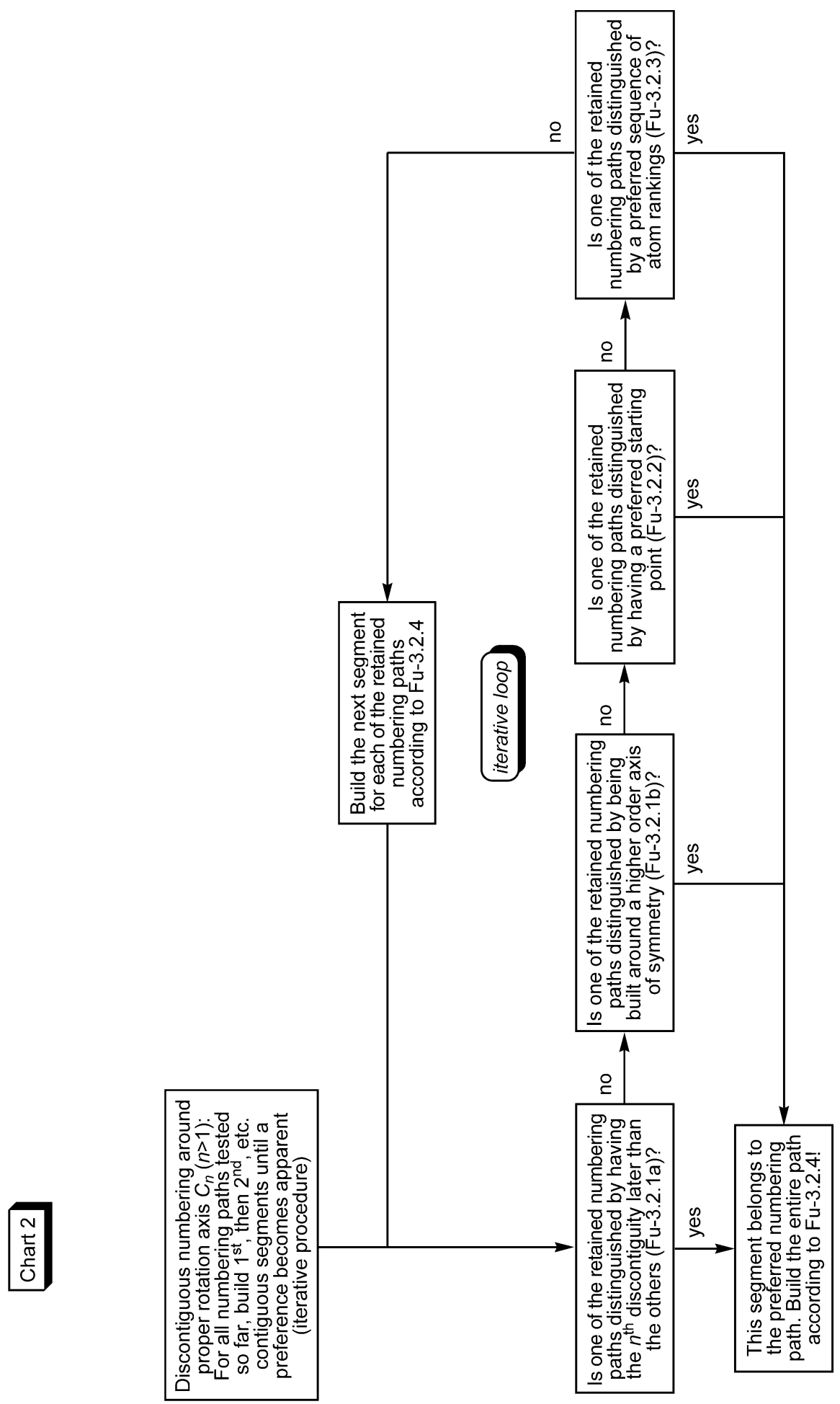

(C) 2005 IUPAC, Pure and Applied Chemistry 77, 843-923 


\section{APPENDIX 3}

In this appendix, the numberings assigned to some fullerenes on the basis of the rules collected in Appendix 1 are reported. Whenever possible, Atlas and CAS Registry Numbers are given. These are the only identifiers that distinguish among fullerenes with the same number of carbon atoms, the same ring size(s), and the same symmetry.

\begin{tabular}{|c|c|c|c|}
\hline Structure & Compound & Atlas no. & CAS reg. no. \\
\hline 1 & $\left({ }^{f, s} C\right)\left(\mathrm{C}_{20}-D_{3}\right)[4,6]$ fullerene & & $182949-12-8$ \\
\hline 2 & $\left({ }^{f, s} A\right)\left(C_{32}-D_{3}\right)[5,6]$ fullerene & $32: 6$ & $115383-20-5$ \\
\hline 3 & $\left(\mathrm{C}_{32}-D_{3 \mathrm{~h}}\right)[5,6]$ fullerene & $32: 5$ & $188937-98-6$ \\
\hline 4 & $\left(\mathrm{C}_{36}-D_{6 \mathrm{~h}}\right)[5,6]$ fullerene & $36: 15$ & $144161-03-5$ \\
\hline 5 & $\left(\mathrm{C}_{38^{-}}-D_{3 \mathrm{~h}}\right)[5,6]$ fullerene & $38: 2$ & $146269-87-6$ \\
\hline 6 & $\left({ }^{f, s} A\right)\left(C_{40}-D_{2}\right)[4,6]$ fullerene & $40: 40$ & $163479-22-9$ \\
\hline 7 & $\left(\mathrm{C}_{40}-T_{\mathrm{d}}\right)[5,6]$ fullerene & & $146269-86-5$ \\
\hline 8 & $\left({ }^{f, s} A\right)\left(\mathrm{C}_{44}-D_{3}\right)[4,6]$ fullerene & & $153415-84-0$ \\
\hline 9 & $\left({ }^{f, s} C\right)\left(\mathrm{C}_{50}-C_{1}\right)[5,6]$ fullerene & $50: 85$ & \\
\hline 10 & $\left(\mathrm{C}_{50}-D_{5 \mathrm{~h}}\right)[5,6]$ fullerene & & $115383-21-6$ \\
\hline 11 & $\left(\mathrm{C}_{60^{-}} C_{2 \mathrm{v}}\right)[4,5,6]$ fullerene & & $150398-38-2$ \\
\hline 12 & $\left(\mathrm{C}_{62}-C_{2 \mathrm{v}}\right)[4,5,6]$ fullerene & & $176249-21-1$ \\
\hline 13 & $\left(\mathrm{C}_{66}-C_{2 \mathrm{v}}\right)[5,6]$ fullerene & & $176249-26-6$ \\
\hline 14 & $\left({ }^{\mathrm{f}, \mathrm{S}} A\right)\left(\mathrm{C}_{68}-D_{3}\right)[5,6]$ fullerene & & $612845-79-1$ \\
\hline 15 & $\left(\mathrm{C}_{70}-C_{\mathrm{s}}\right)[5,6]$ fullerene & & $186821-65-8$ \\
\hline 16 & $\left(\mathrm{C}_{74}-D_{3 \mathrm{~h}}\right)[5,6]$ fullerene & 74:1 & $147398-93-4$ \\
\hline 17 & $\left(\mathrm{C}_{78}-D_{3 \mathrm{~h}}\right)[5,6]$ fullerene & 78:5 & $145954-03-6$ \\
\hline 18 & $\left(\mathrm{C}_{78}-D_{3 \mathrm{~h}}\right)[5,6]$ fullerene & $78: 4$ & $145392-90-1$ \\
\hline 19 & $\left({ }^{\mathrm{f}, \mathrm{S}} \mathrm{C}\right)\left(\mathrm{C}_{\left.80^{-} D_{2}\right)[5,6] \text { fullerene }}\right.$ & $80: 2$ & $147230-75-9$ \\
\hline 20 & $\left(\mathrm{C}_{80}-D_{5 \mathrm{~d}}\right)[5,6]$ fullerene & $80: 1$ & $145990-38-1$ \\
\hline 21 & $\left(\mathrm{C}_{80}-D_{5 \mathrm{~h}}\right)[5,6]$ fullerene & $80: 6$ & $133947-15-6$ \\
\hline 22 & $\left({ }^{f, s} A\right)\left(C_{82}-C_{2}\right)[5,6]$ fullerene & $82: 3$ & $145954-04-7$ \\
\hline 23 & $\left(\mathrm{C}_{\left.82^{-} C_{\mathrm{s}}\right)[5,6] \text { fullerene }}\right.$ & $82: 6$ & $147230-78-2$ \\
\hline 24 & $\left(\mathrm{C}_{82}-C_{2 \mathrm{y}}\right)[5,6]$ fullerene & $82: 9$ & $141049-97-0$ \\
\hline 25 & $\left(\mathrm{C}_{82}-C_{3 \mathrm{v}}\right)[5,6]$ fullerene & $82: 7$ & $144274-33-9$ \\
\hline 26 & $\left({ }^{f, s} A\right)\left(C_{84}-D_{2}\right)[5,6]$ fullerene & $84: 22$ & $145809-19-4$ \\
\hline 27 & $\left(\mathrm{C}_{84}-D_{2 \mathrm{~d}}\right)[5,6]$ fullerene & $84: 4$ & $145809-03-6$ \\
\hline 28 & $\left(\mathrm{C}_{84}-D_{3 \mathrm{~d}}\right)[5,6]$ fullerene & $84: 19$ & $145809-17-2$ \\
\hline 29 & $\left(\mathrm{C}_{84}^{\mathrm{ot}}-T_{\mathrm{d}}\right)[5,6]$ fullerene & $84: 20$ & $134847-08-8$ \\
\hline 30 & $\left({ }^{\mathrm{f}, \mathrm{S}} A\right)\left(\mathrm{C}_{\left.86^{-} C_{2}\right)[5,6] \text { fullerene }}\right.$ & $86: 17$ & $182198-51-2$ \\
\hline 31 & $\left(\mathrm{C}_{86}-C_{\mathrm{s}}\right)[5,6]$ fullerene & $86: 16$ & $182198-64-7$ \\
\hline 32 & $\left({ }^{\mathrm{f}, \mathrm{s}} A\right)\left(\mathrm{C}_{88^{-}} C_{2}\right)[5,6]$ fullerene & 88:07 & \\
\hline 33 & $\left({ }^{\mathrm{f}, \mathrm{s}} C\right)\left(\mathrm{C}_{88^{-}} C_{2}\right)[5,6]$ fullerene & $88: 33$ & $183057-05-8$ \\
\hline 34 & $\left(\mathrm{C}_{88^{-}} C_{\mathrm{s}}\right)[5,6]$ fullerene & 88:17 & $183057-33-2$ \\
\hline 35 & $\left({ }^{\mathrm{f}, \mathrm{S}} \mathrm{O}\right)\left(\mathrm{C}_{92}-T\right)[5,6]$ fullerene & $92: 86$ & $158473-42-8$ \\
\hline
\end{tabular}



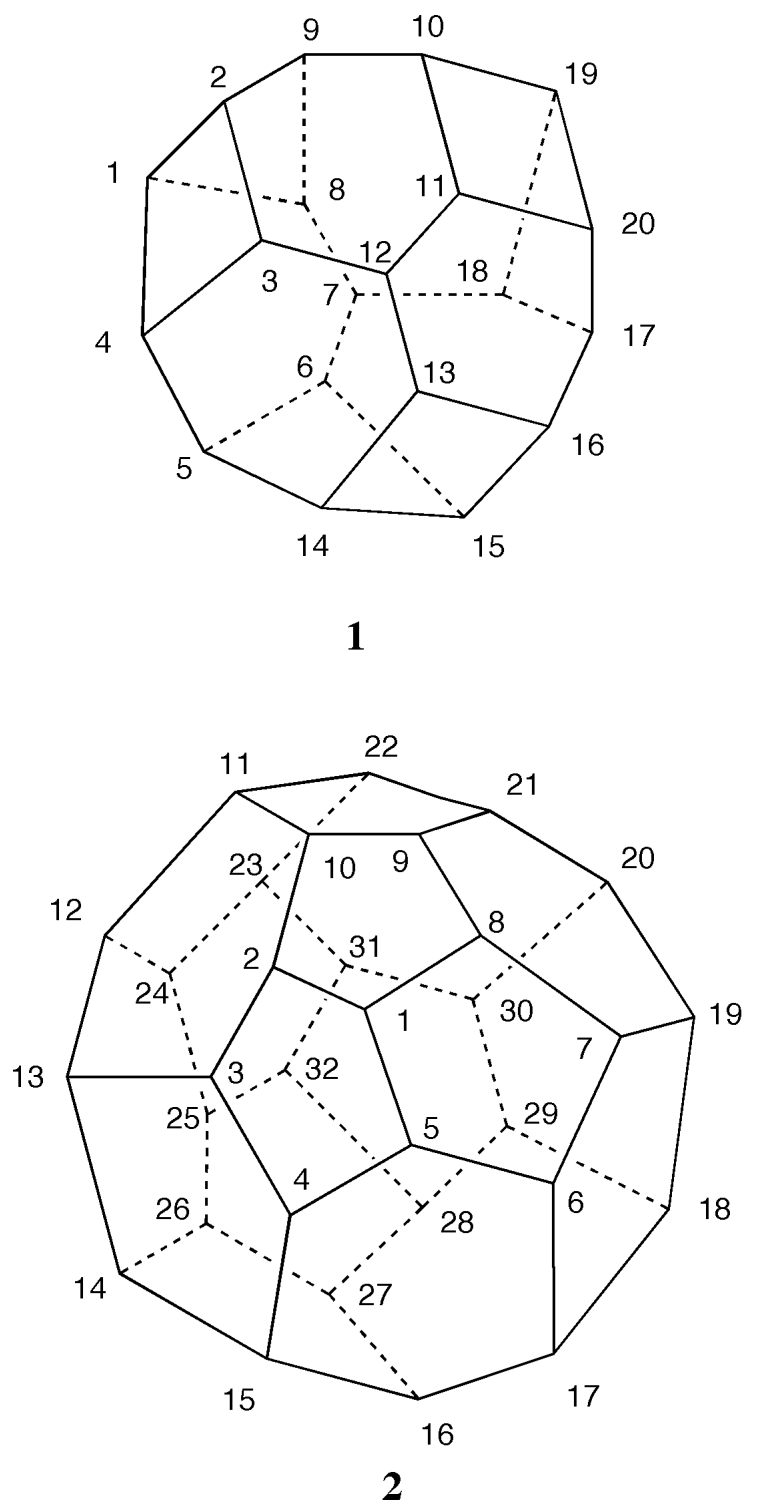

(C) 2005 IUPAC, Pure and Applied Chemistry 77, 843-923 


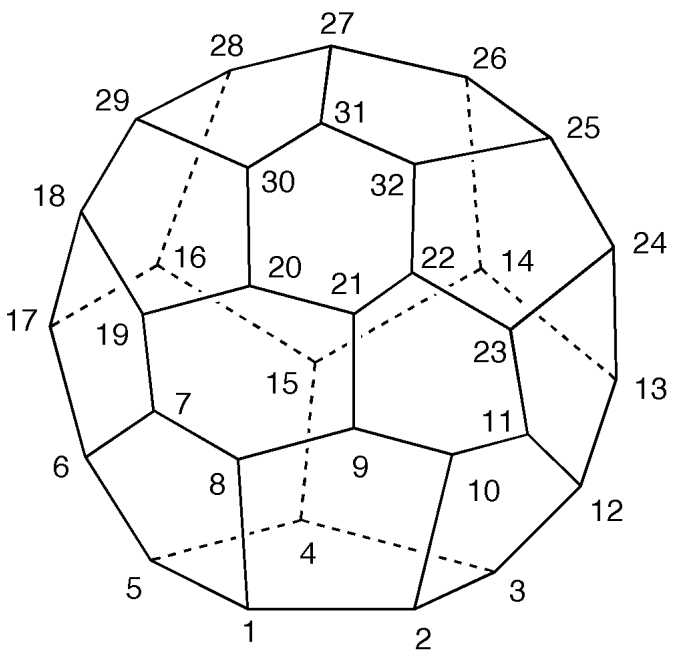

3

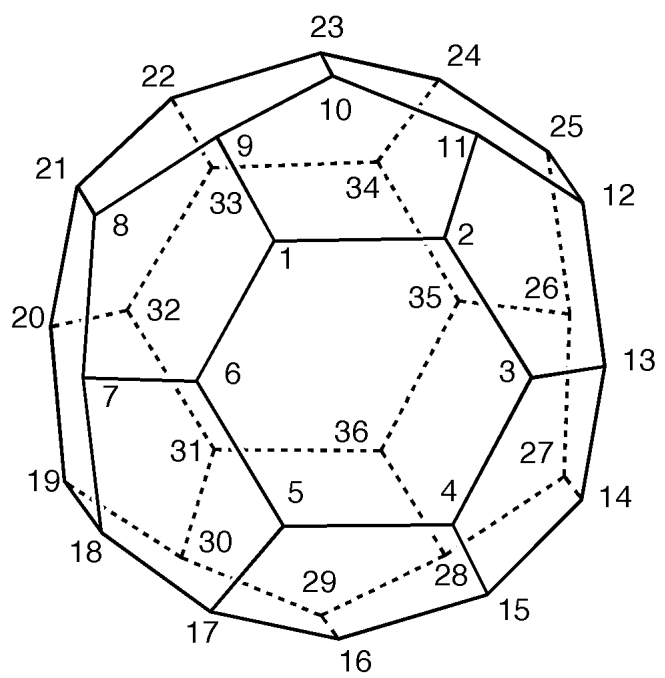

4 

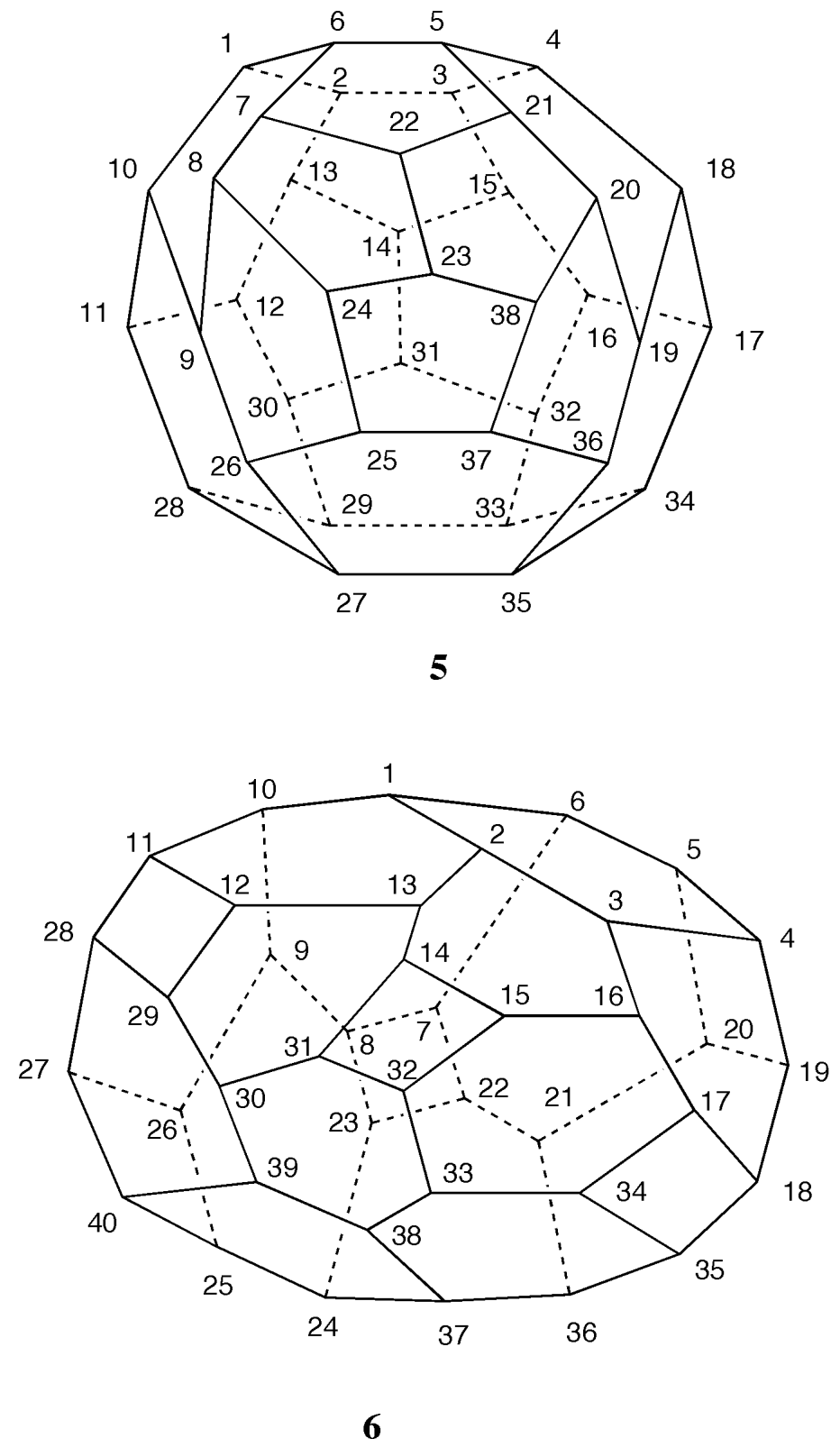

(C) 2005 IUPAC, Pure and Applied Chemistry 77, 843-923 

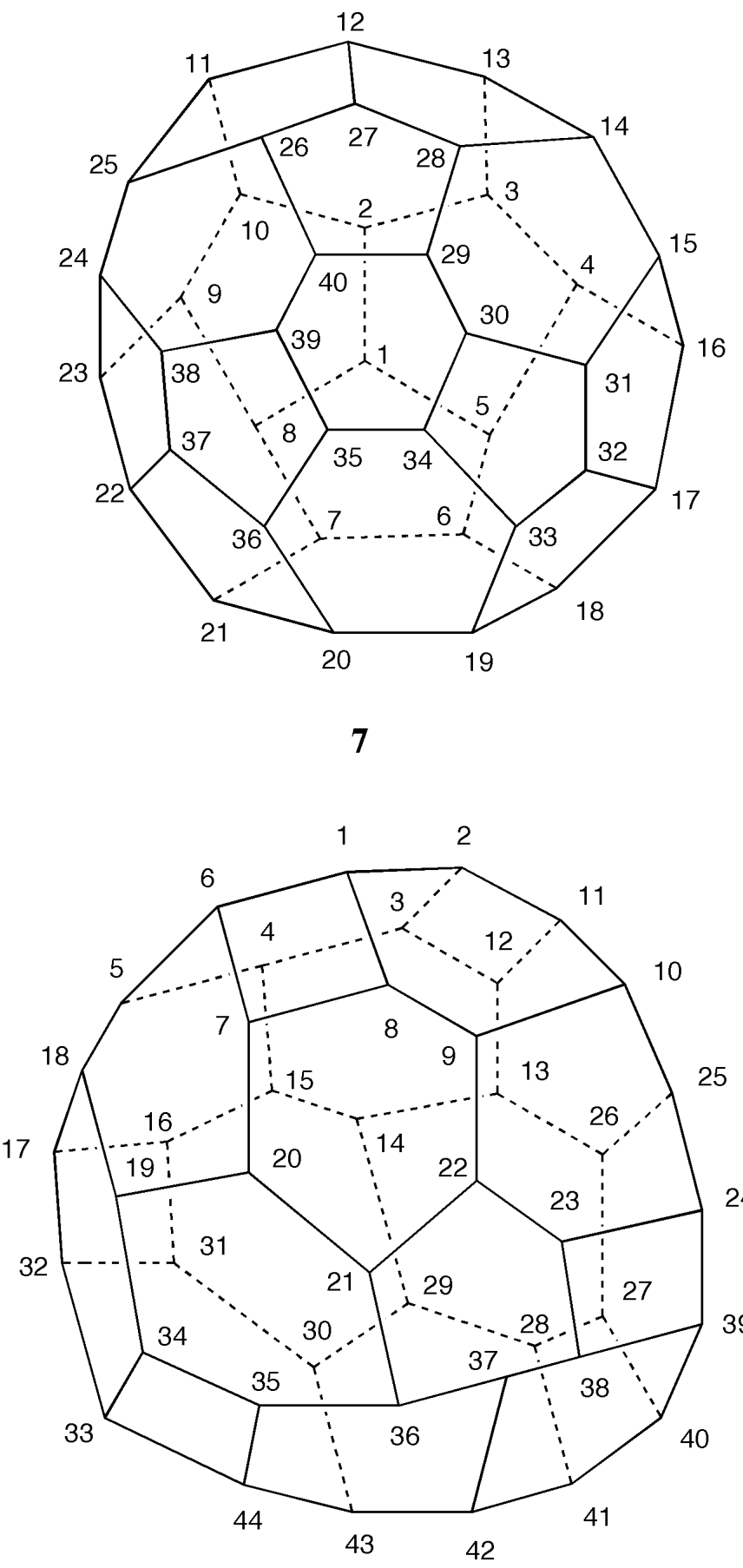

8 


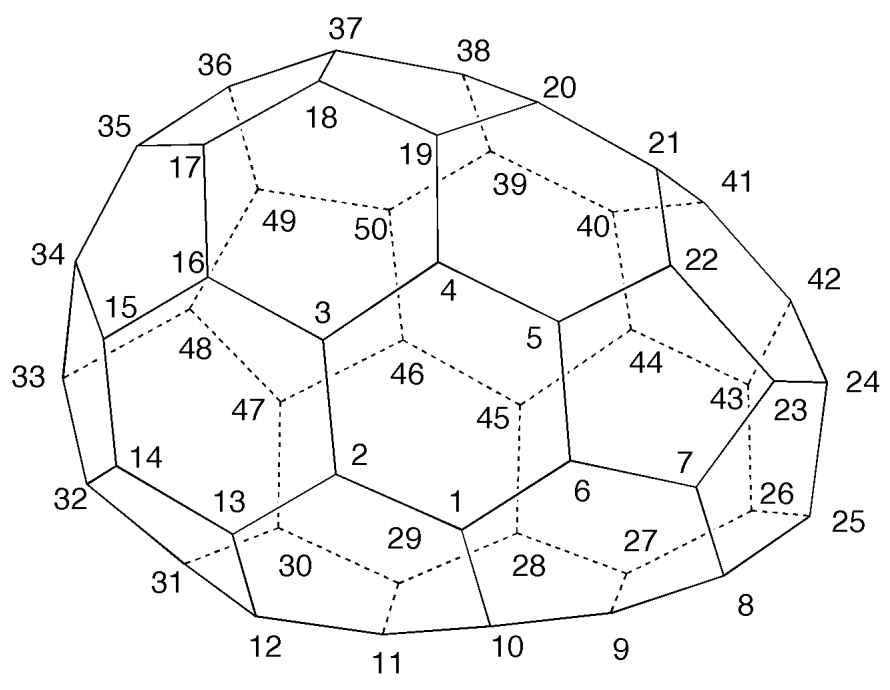

9

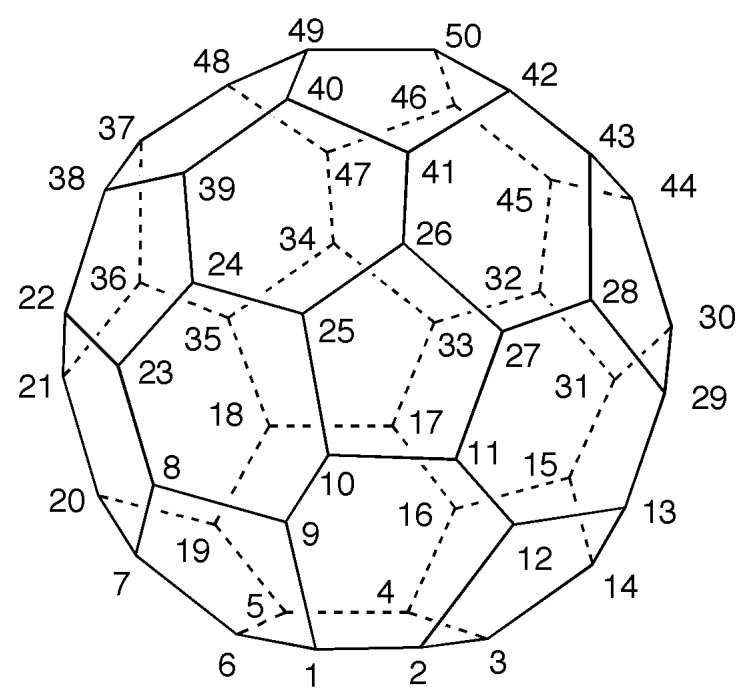

10

(C) 2005 IUPAC, Pure and Applied Chemistry 77, 843-923 


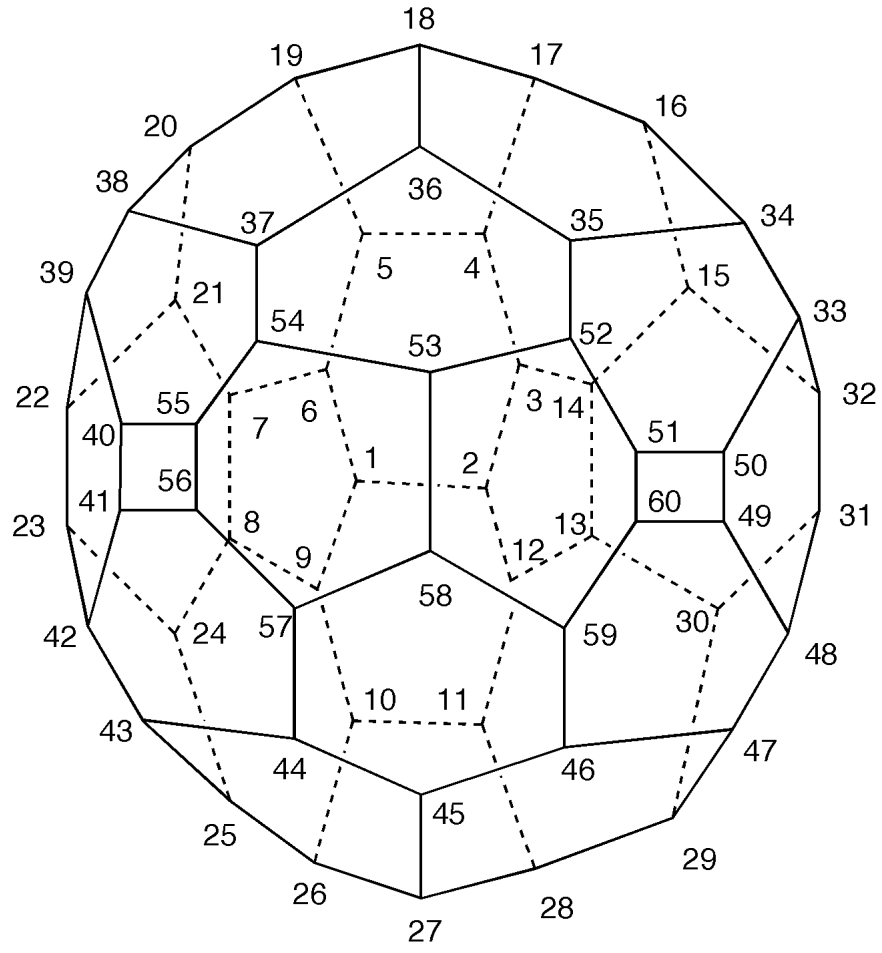

11

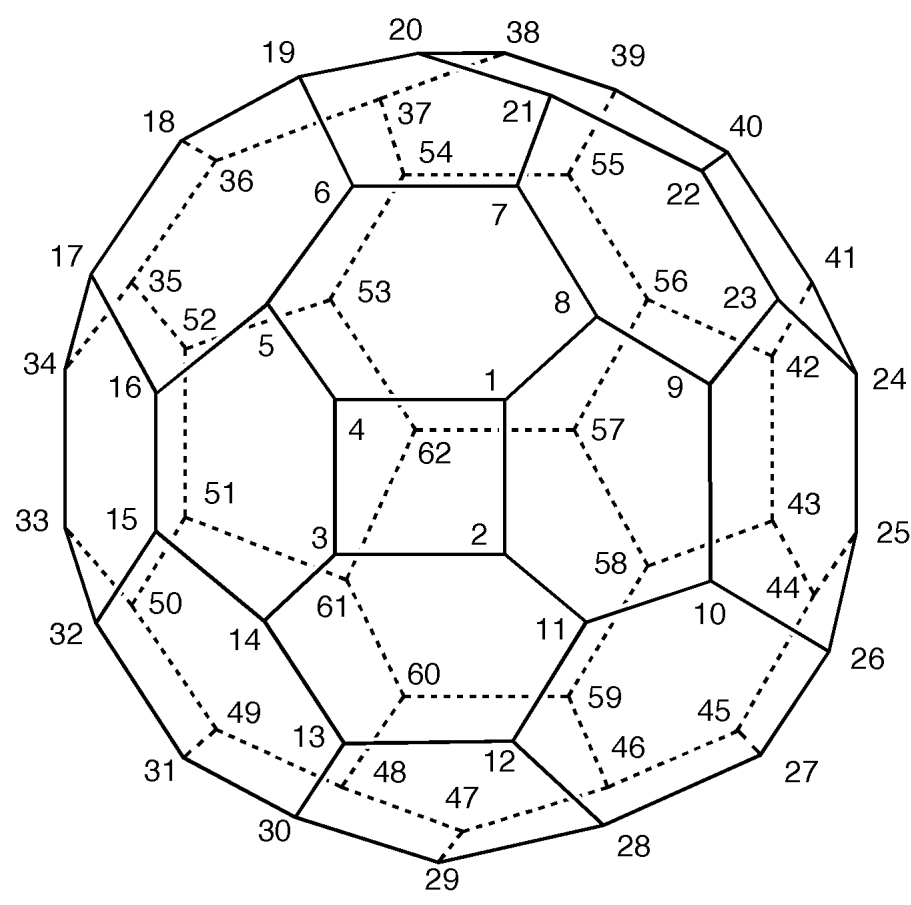

12

(C) 2005 IUPAC, Pure and Applied Chemistry 77, 843-923 


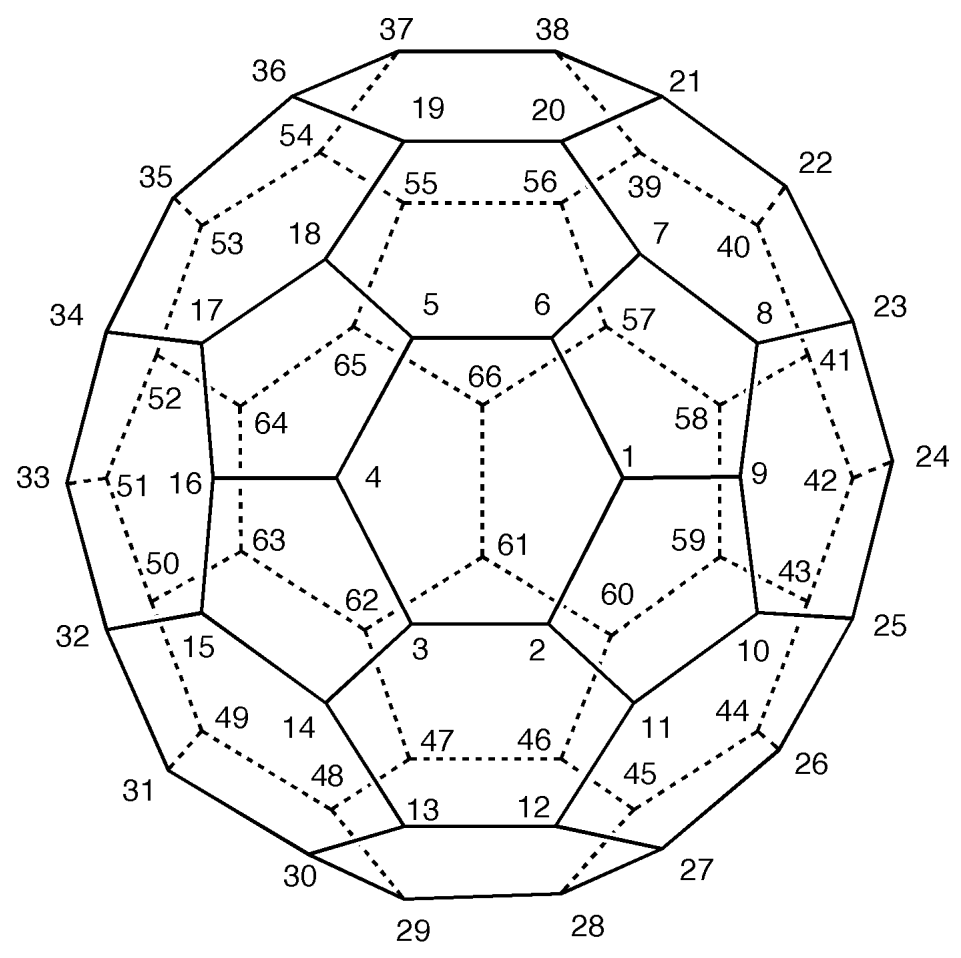

13 


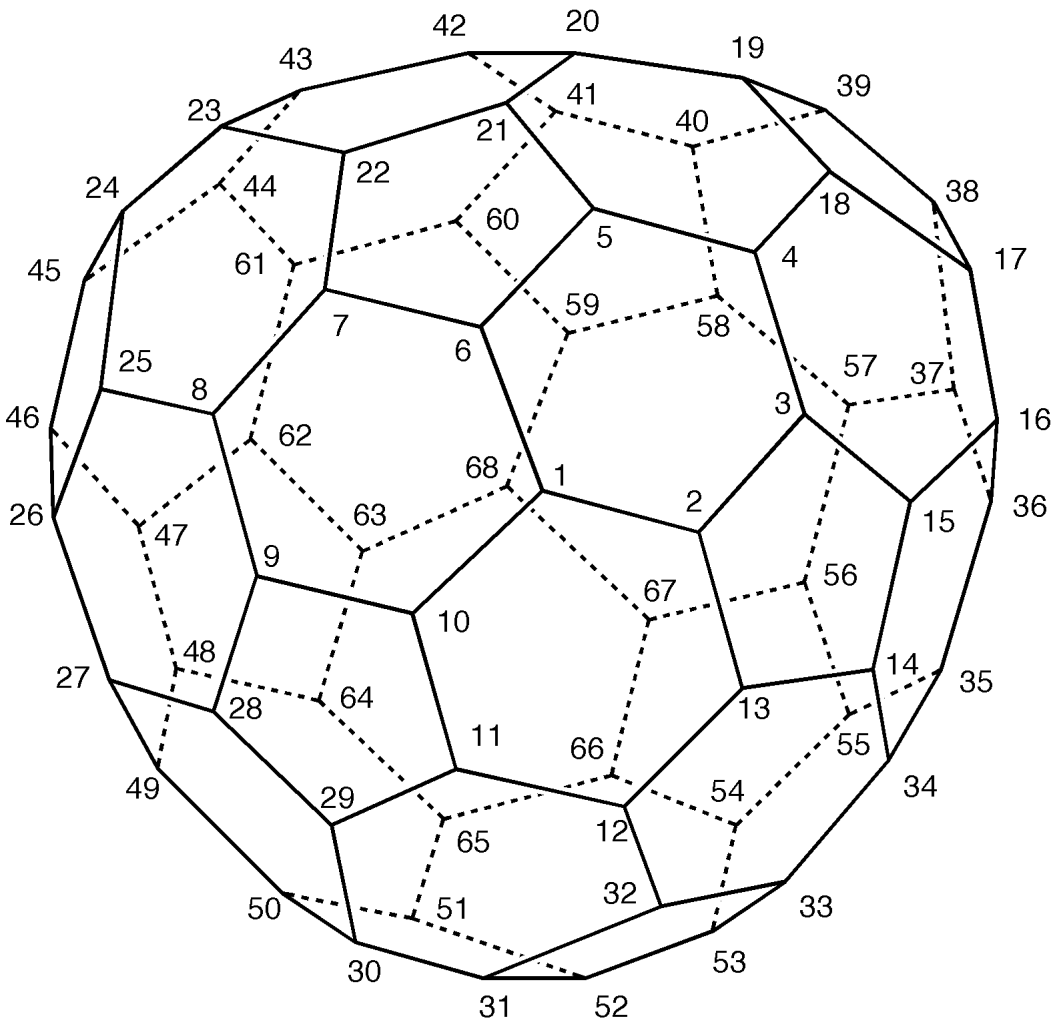

14 


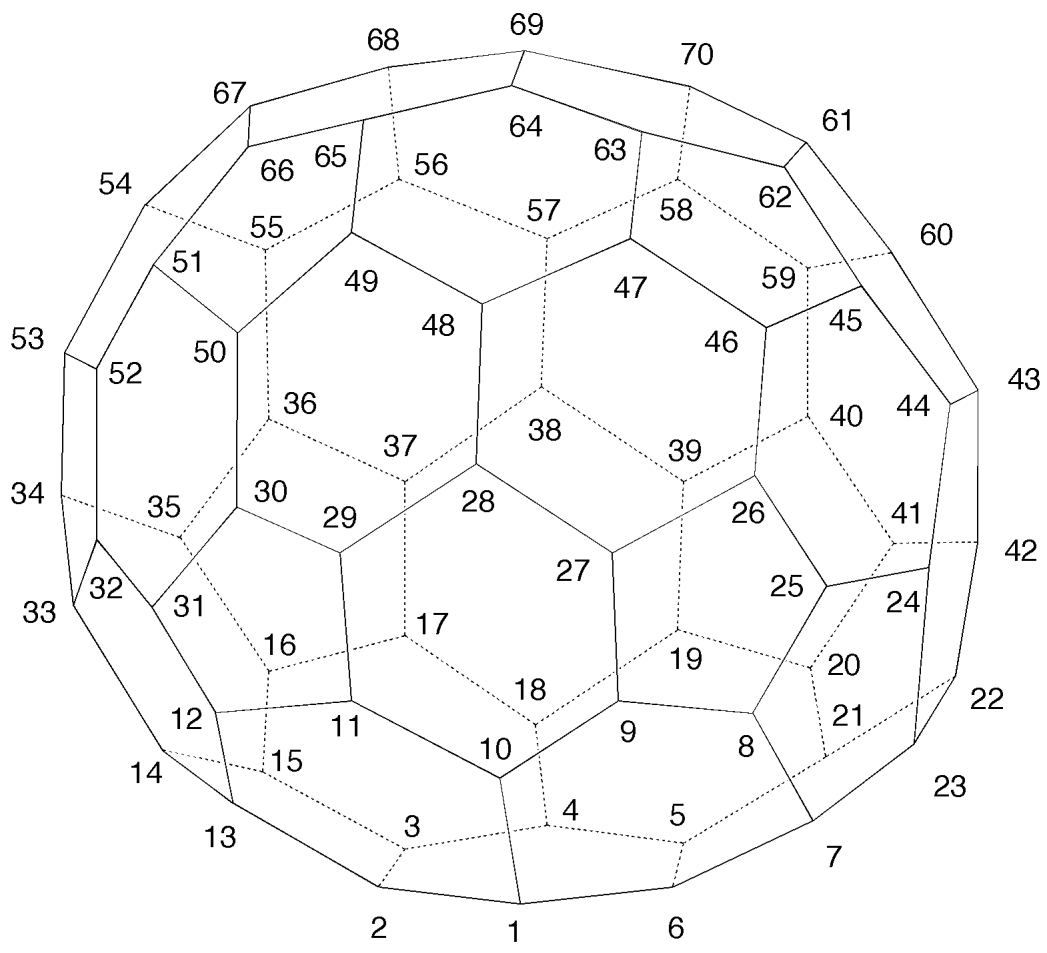

15 


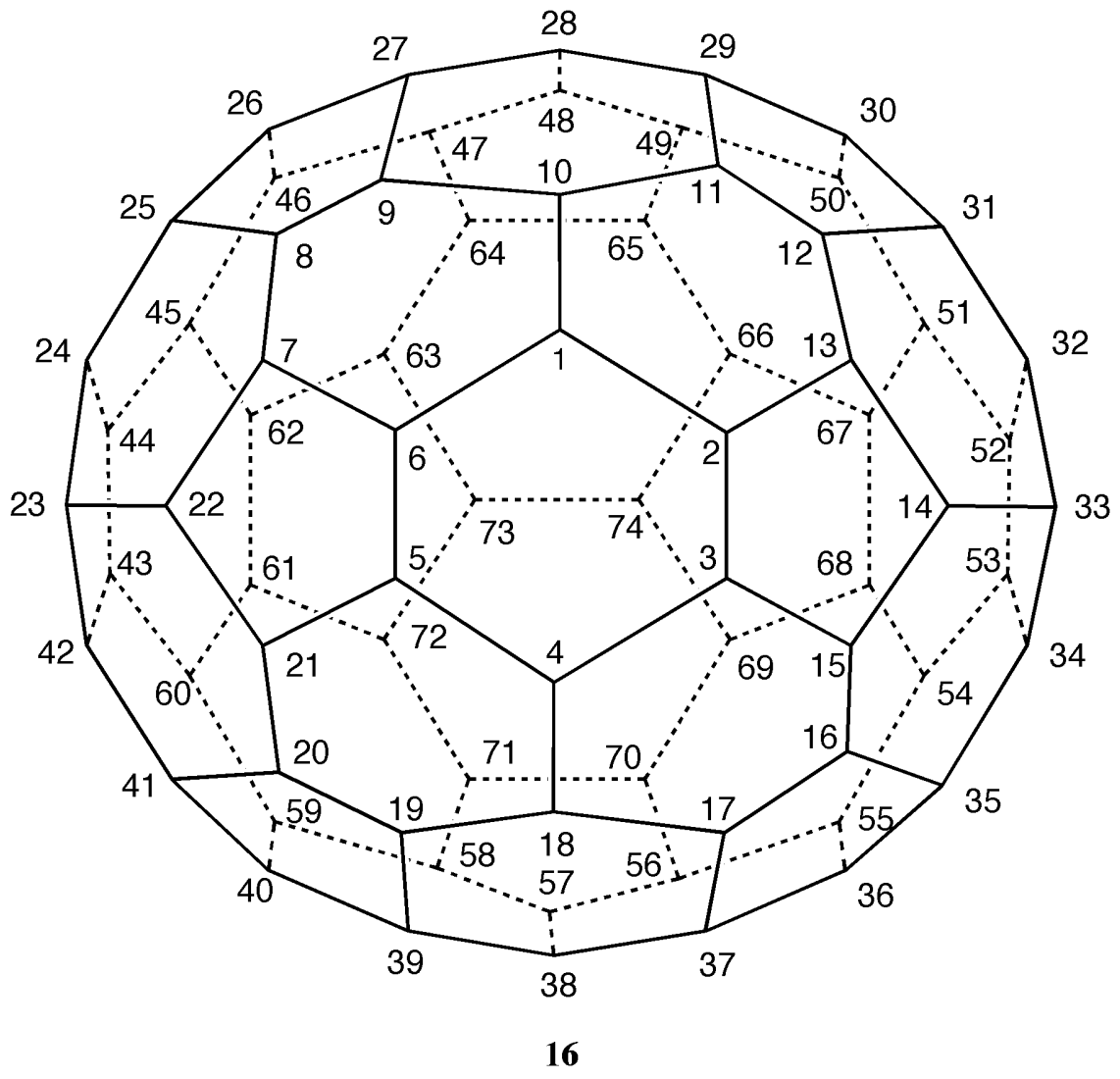




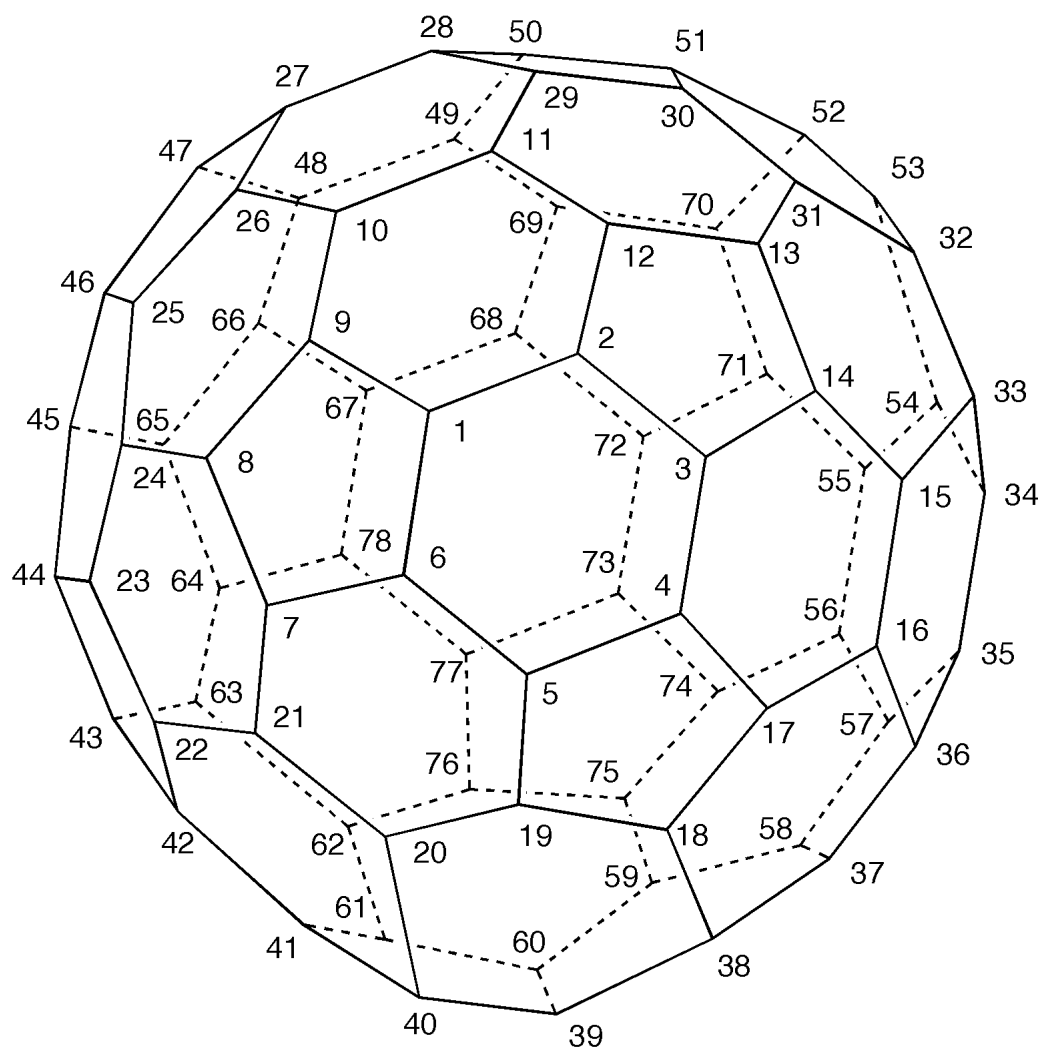

17 


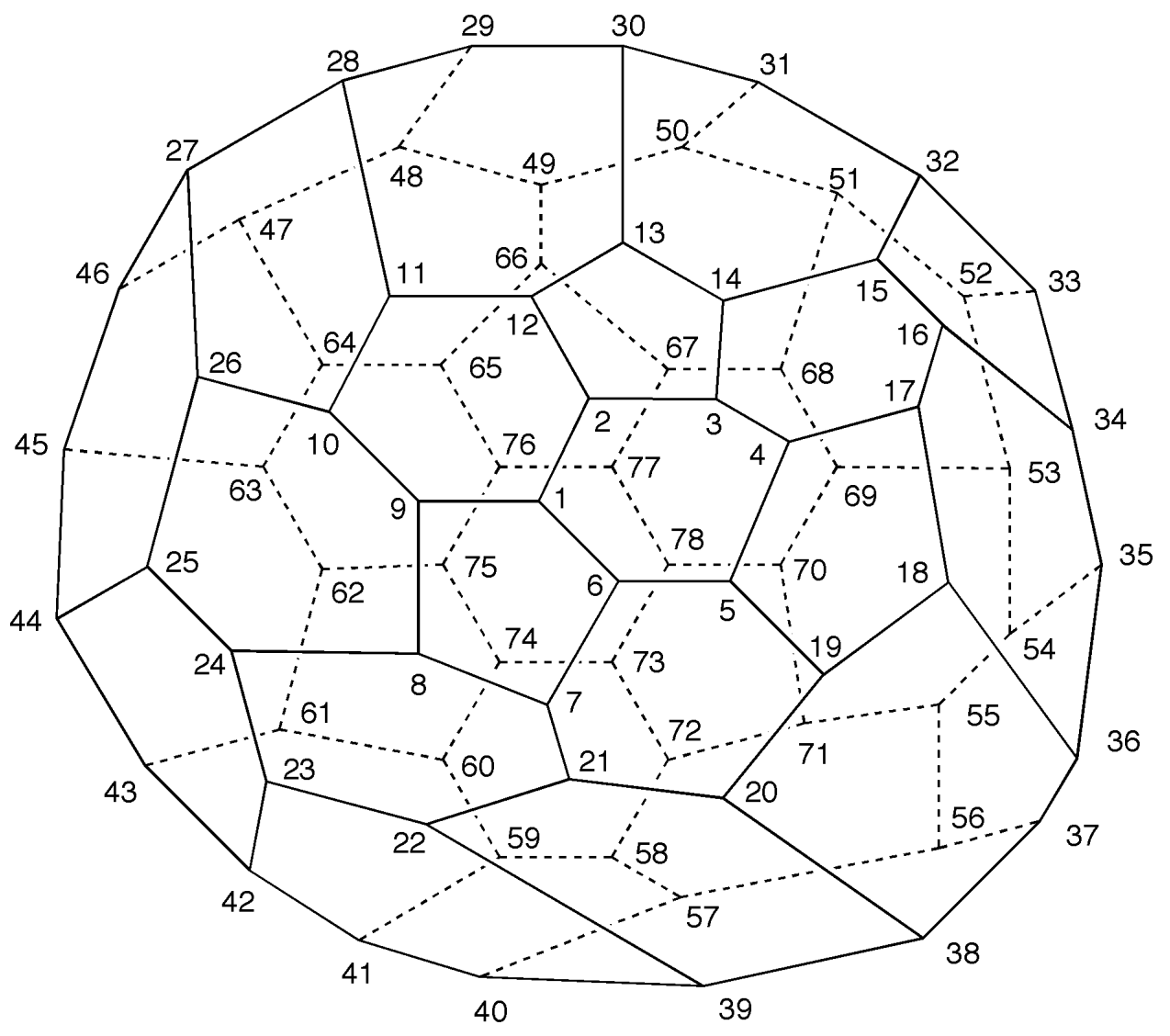

18 


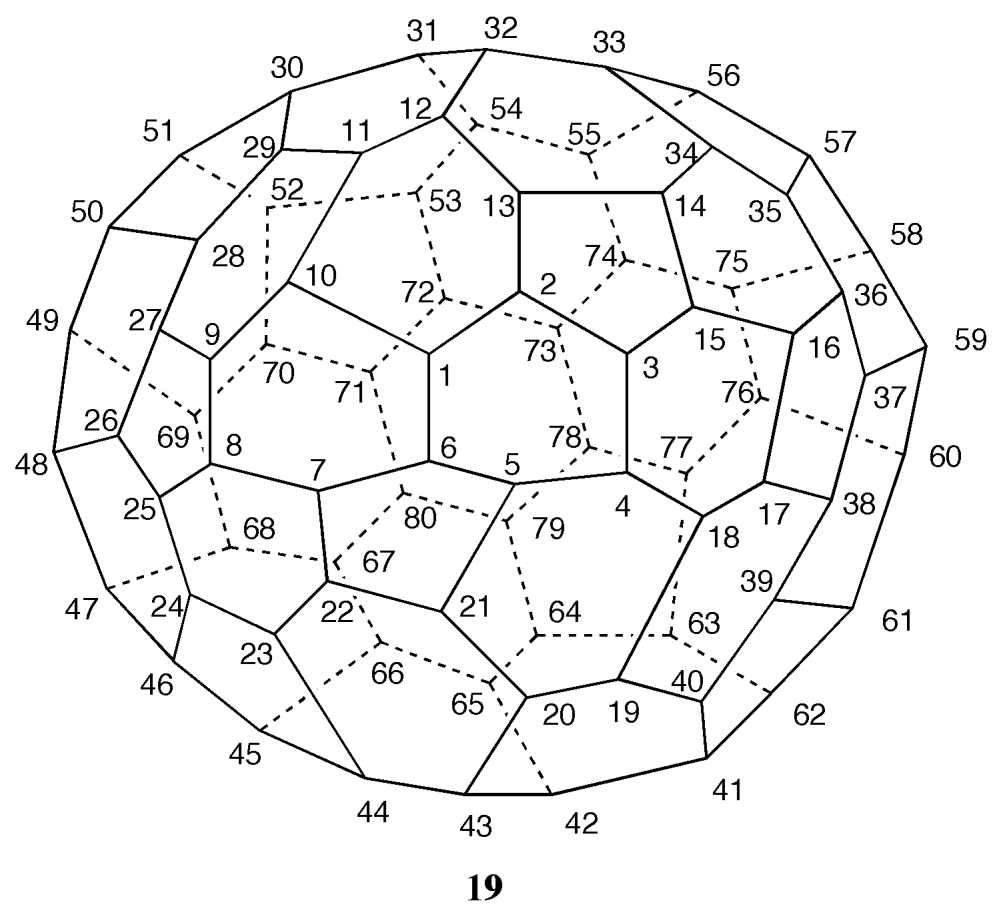




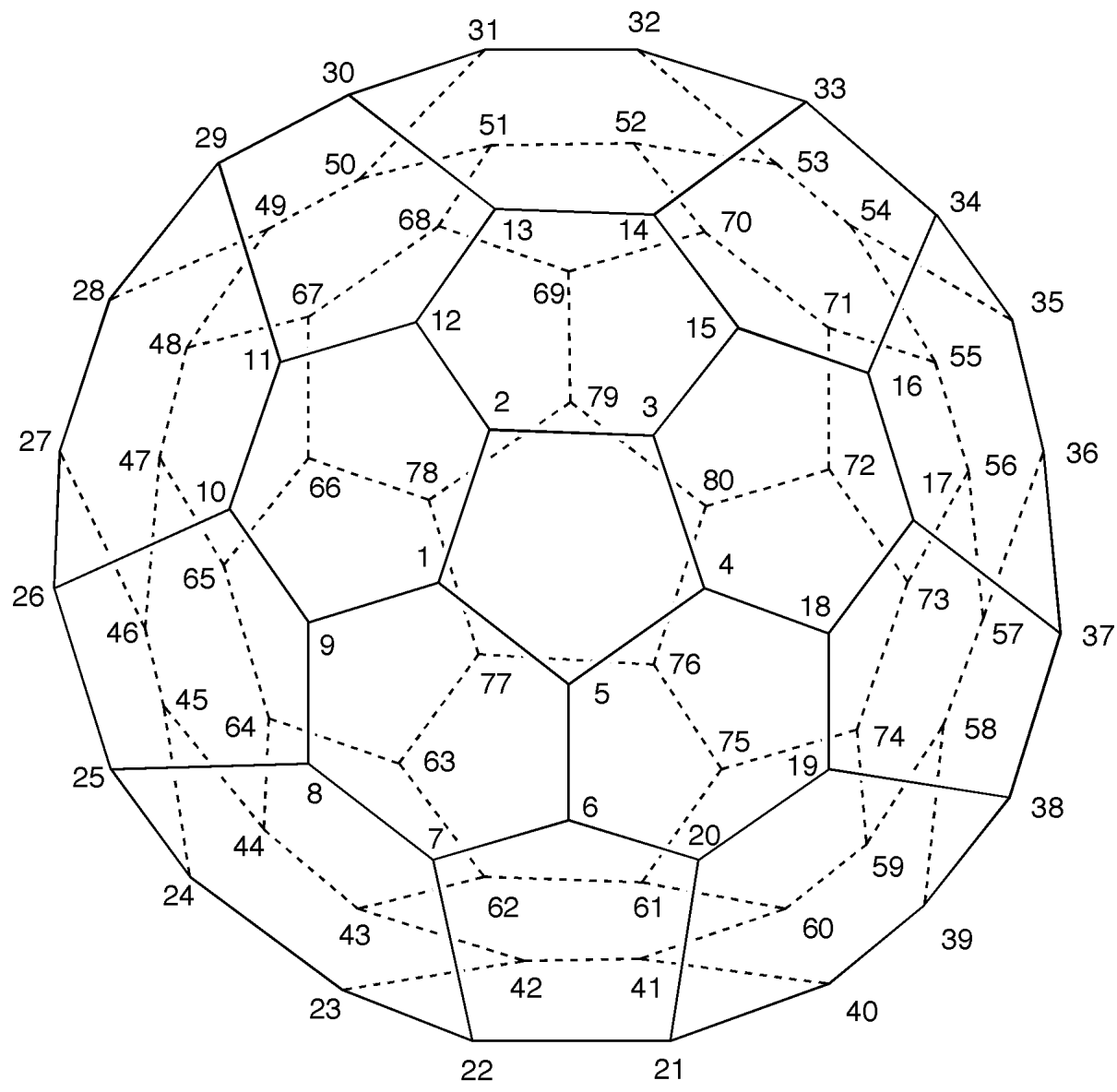




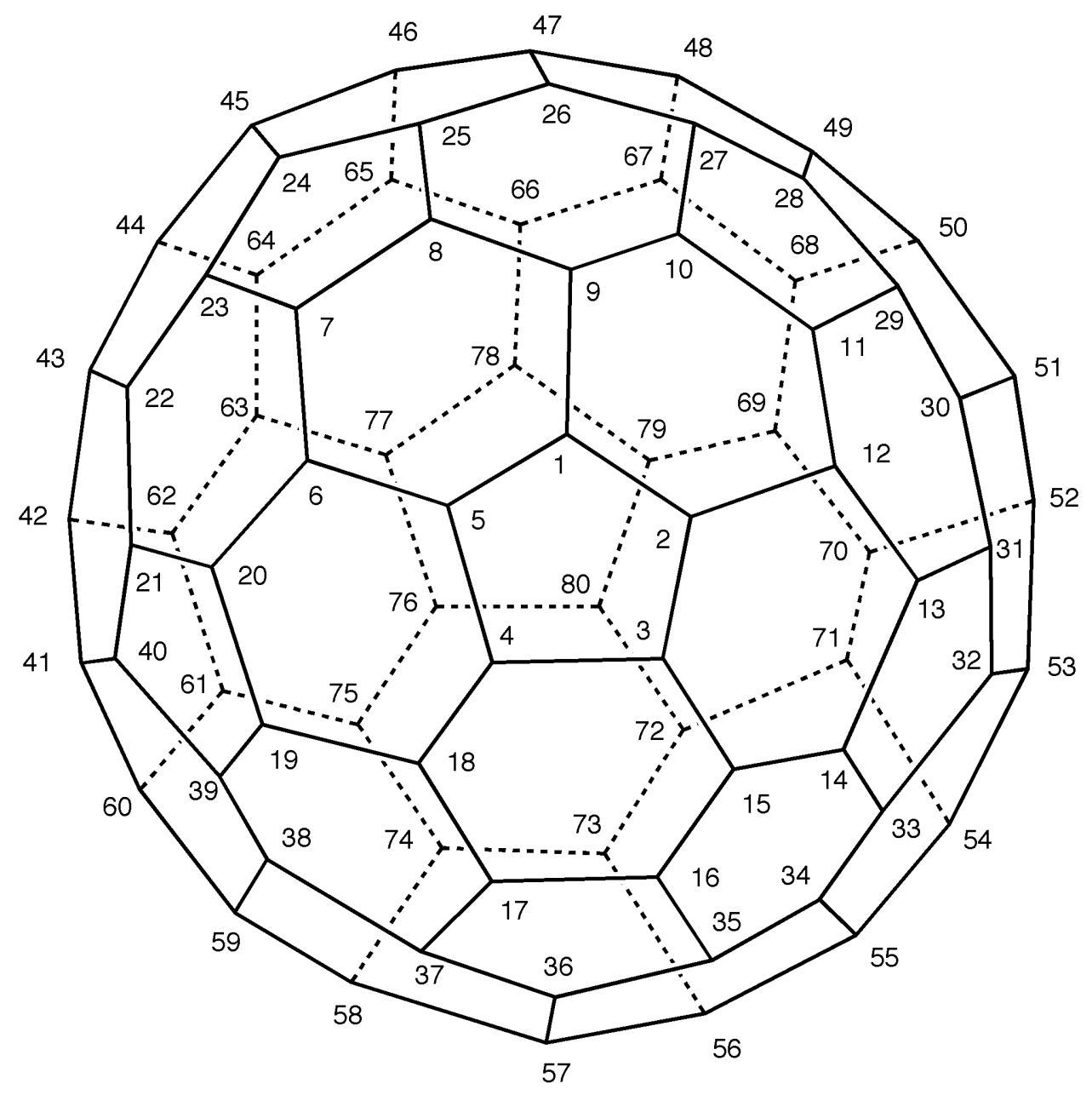




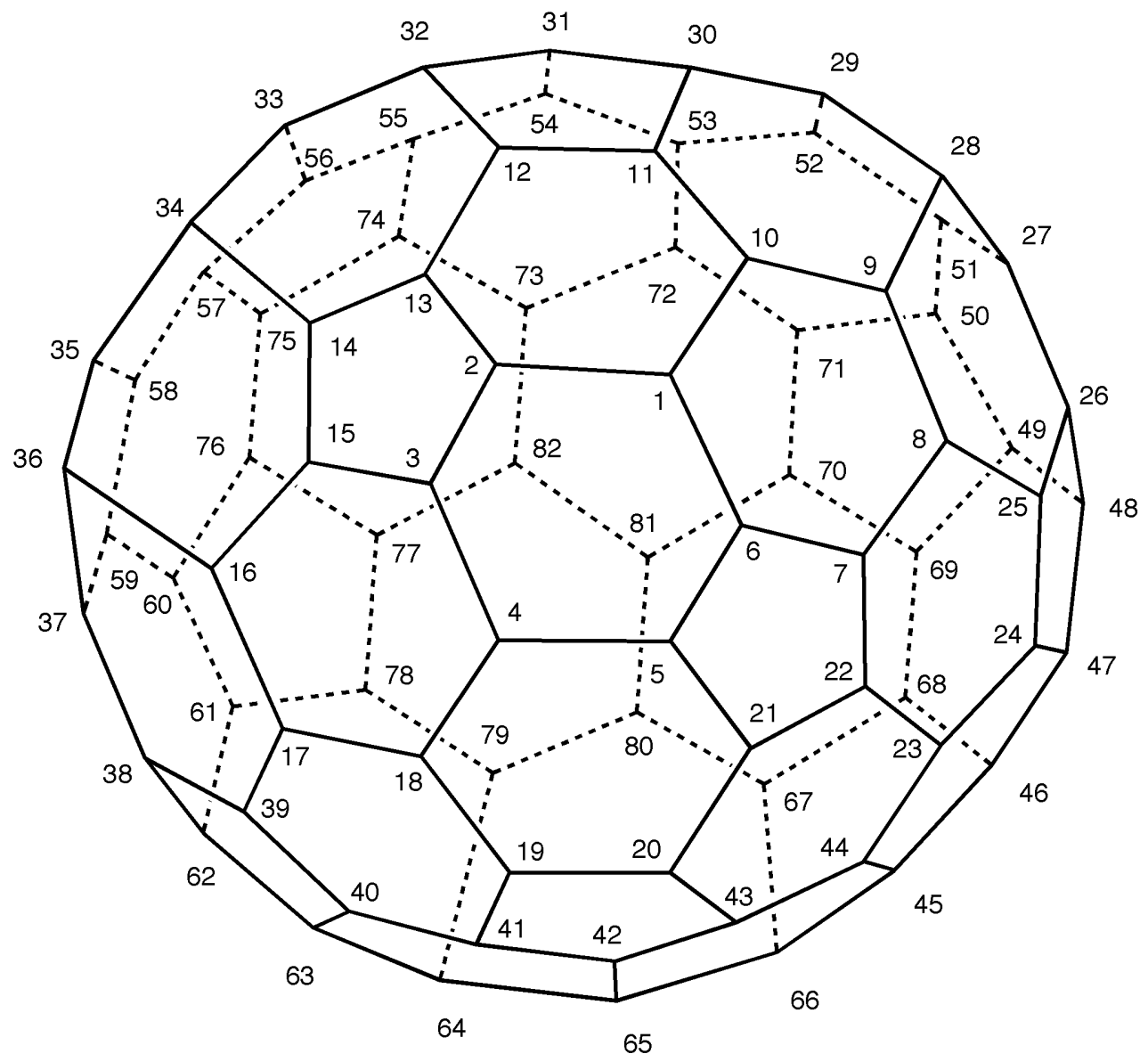




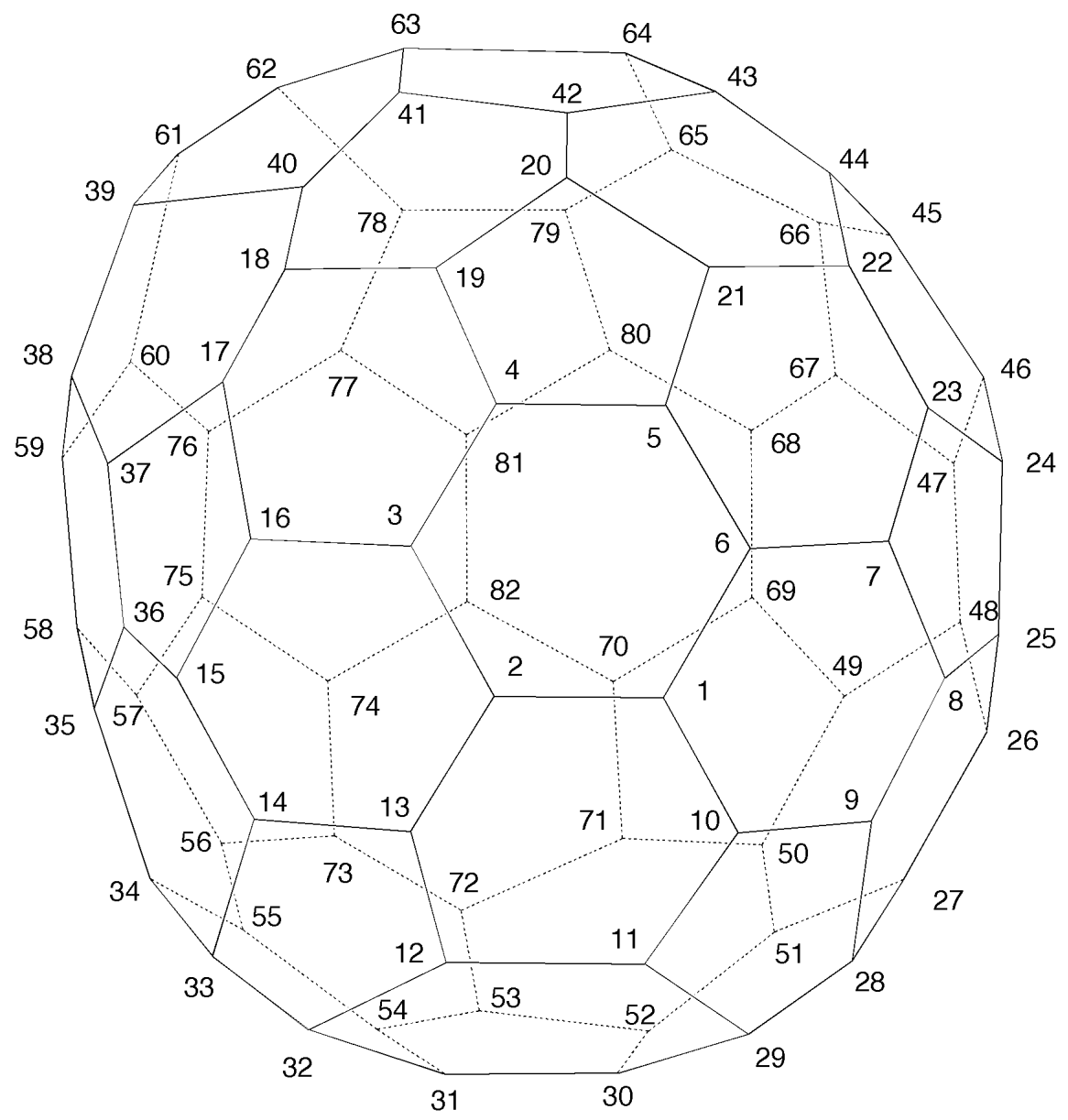

23

(C) 2005 IUPAC, Pure and Applied Chemistry 77, 843-923 


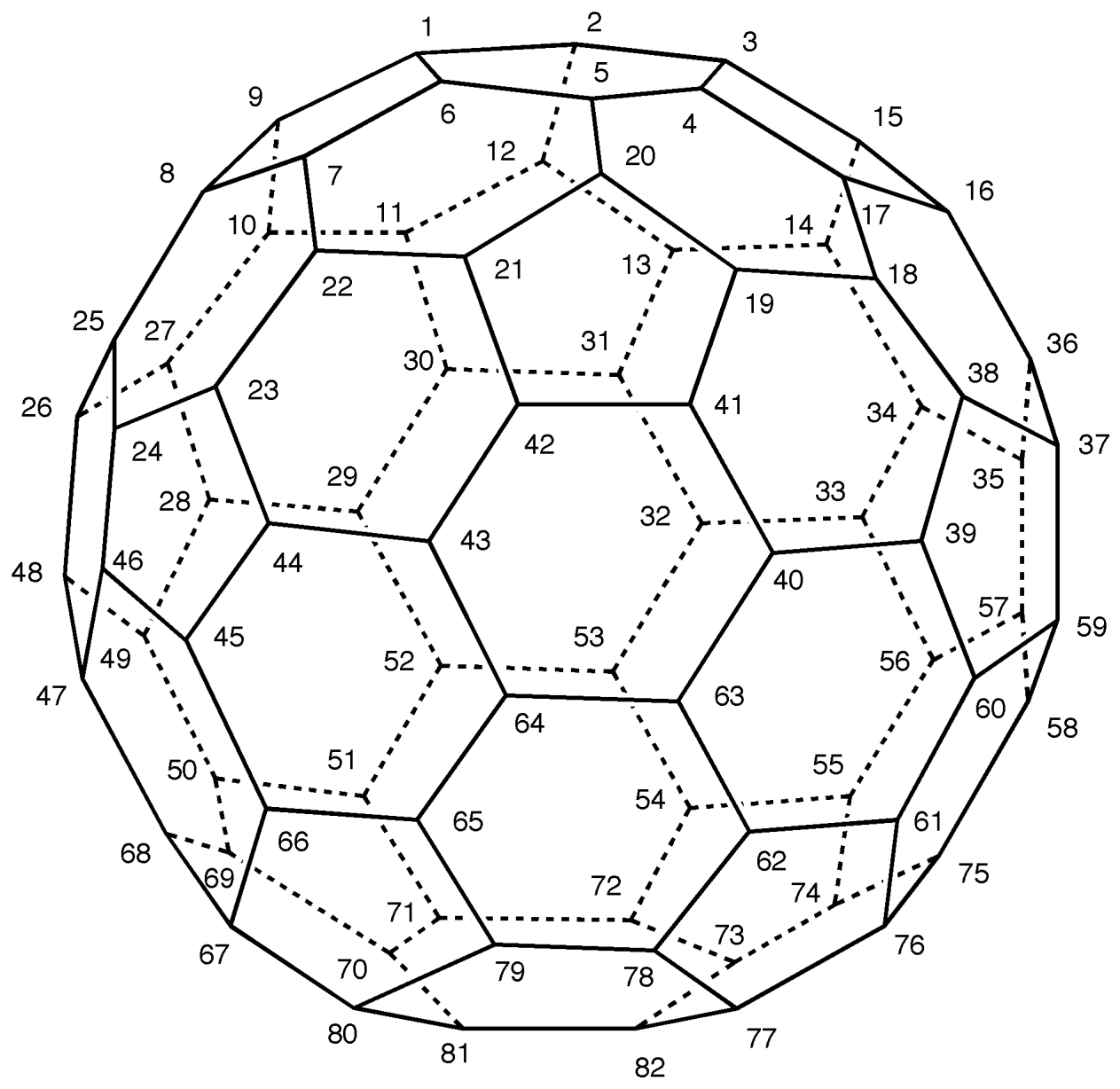




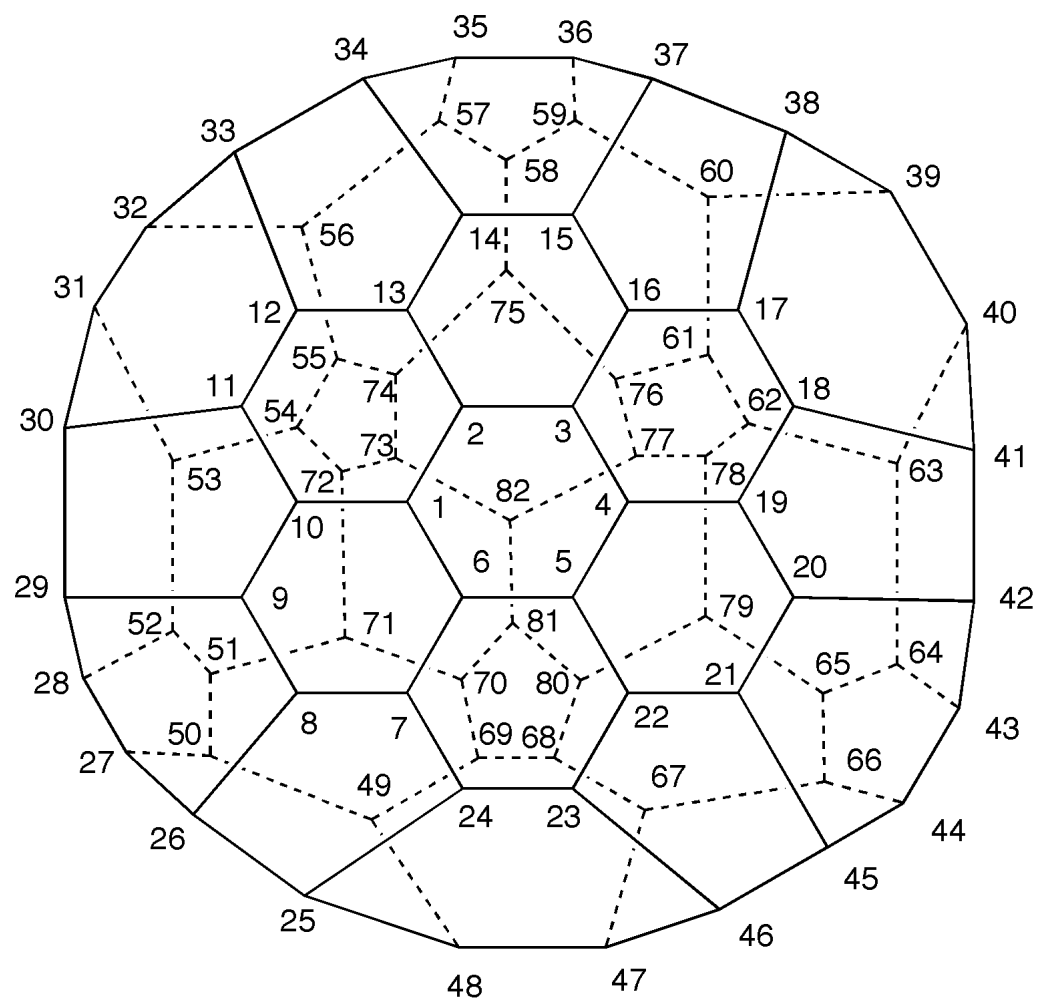

25 


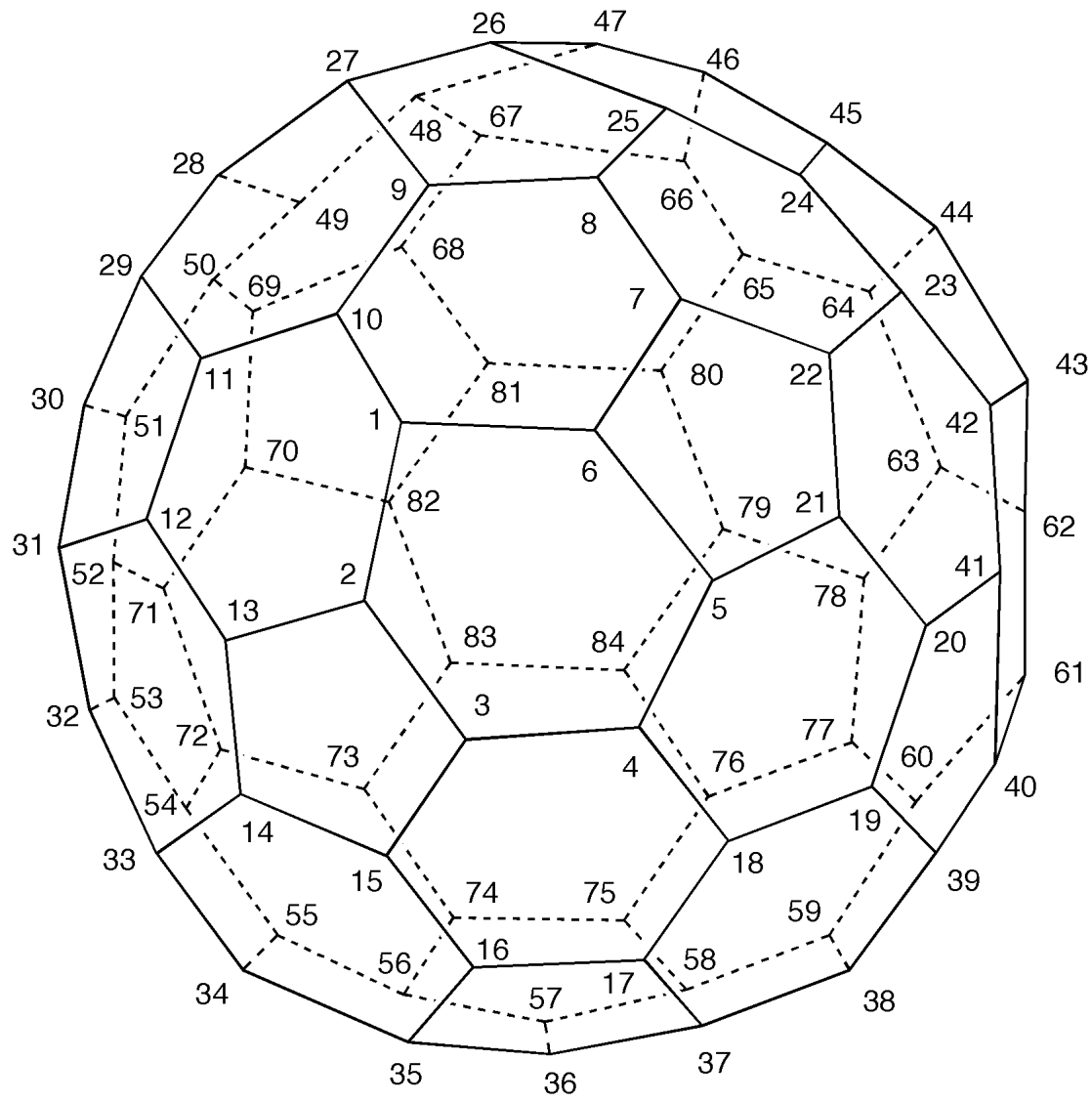

26 

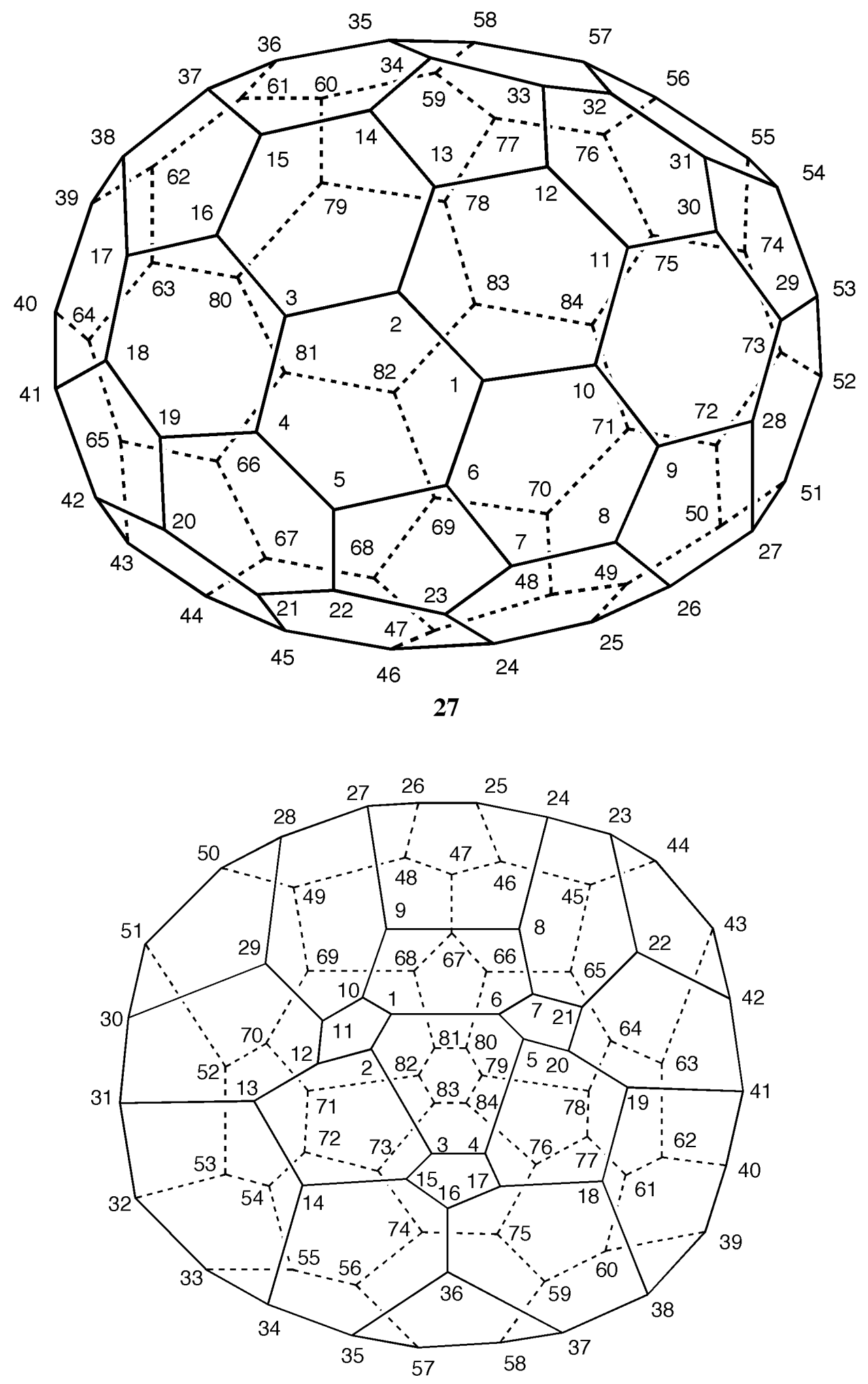

28

(C) 2005 IUPAC, Pure and Applied Chemistry 77, 843-923 


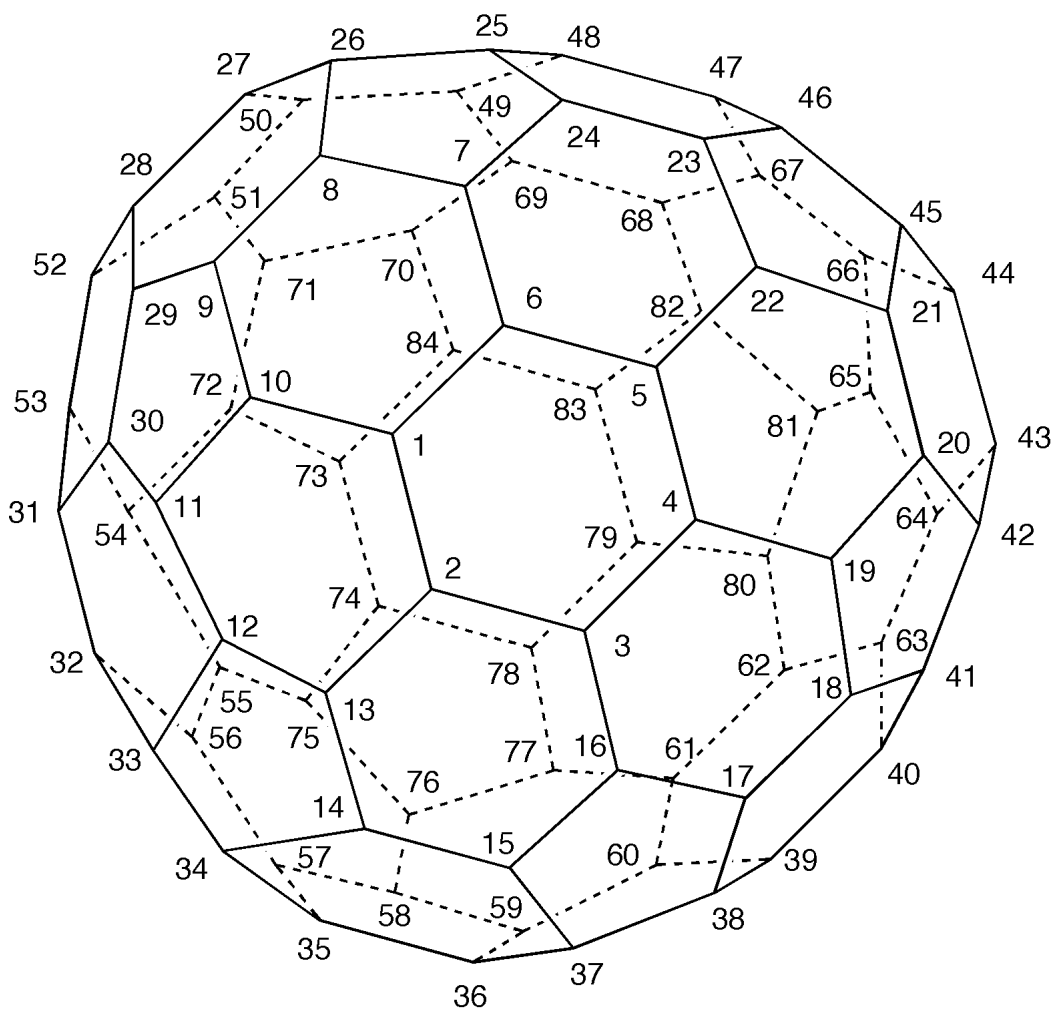

29 


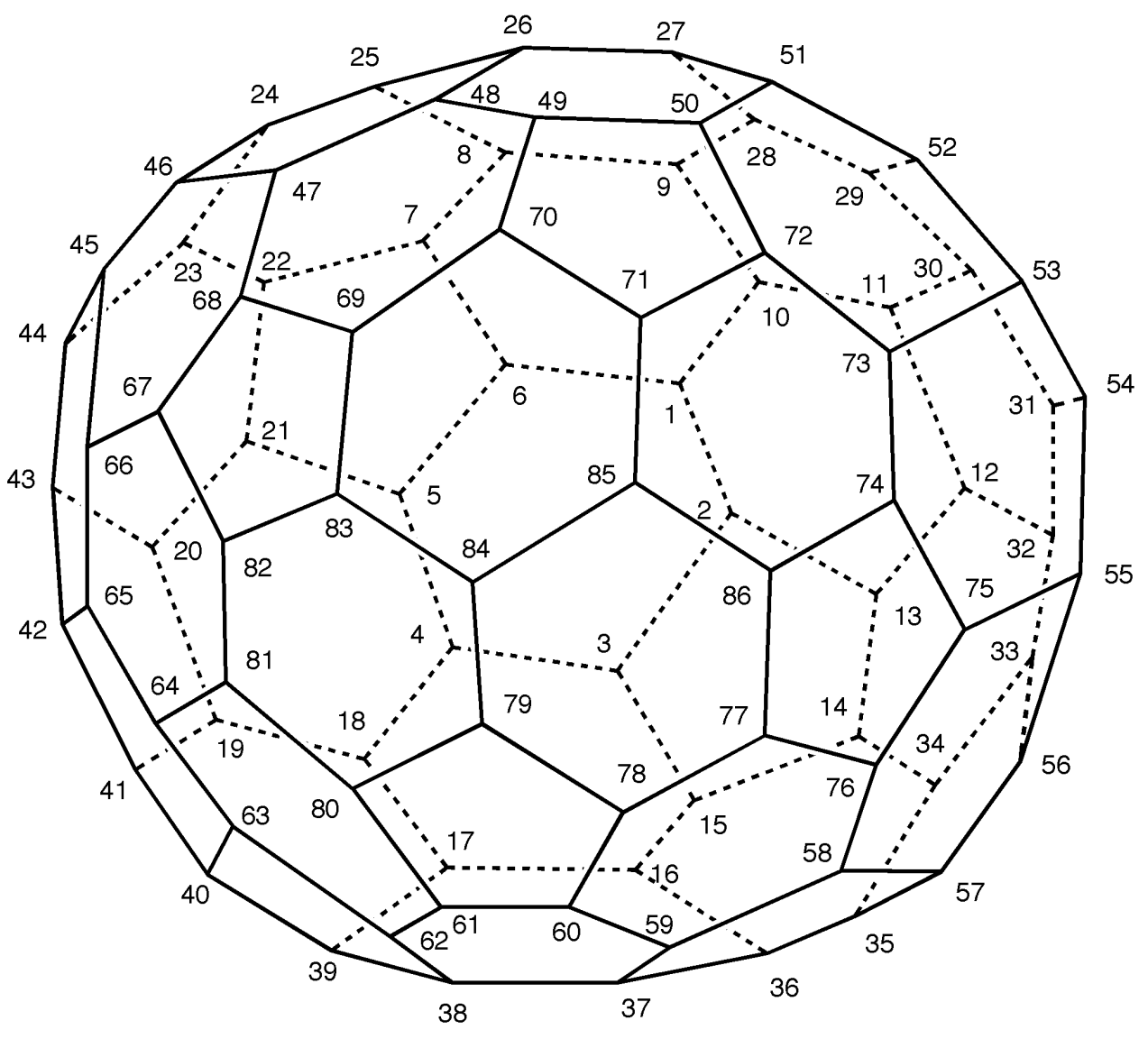




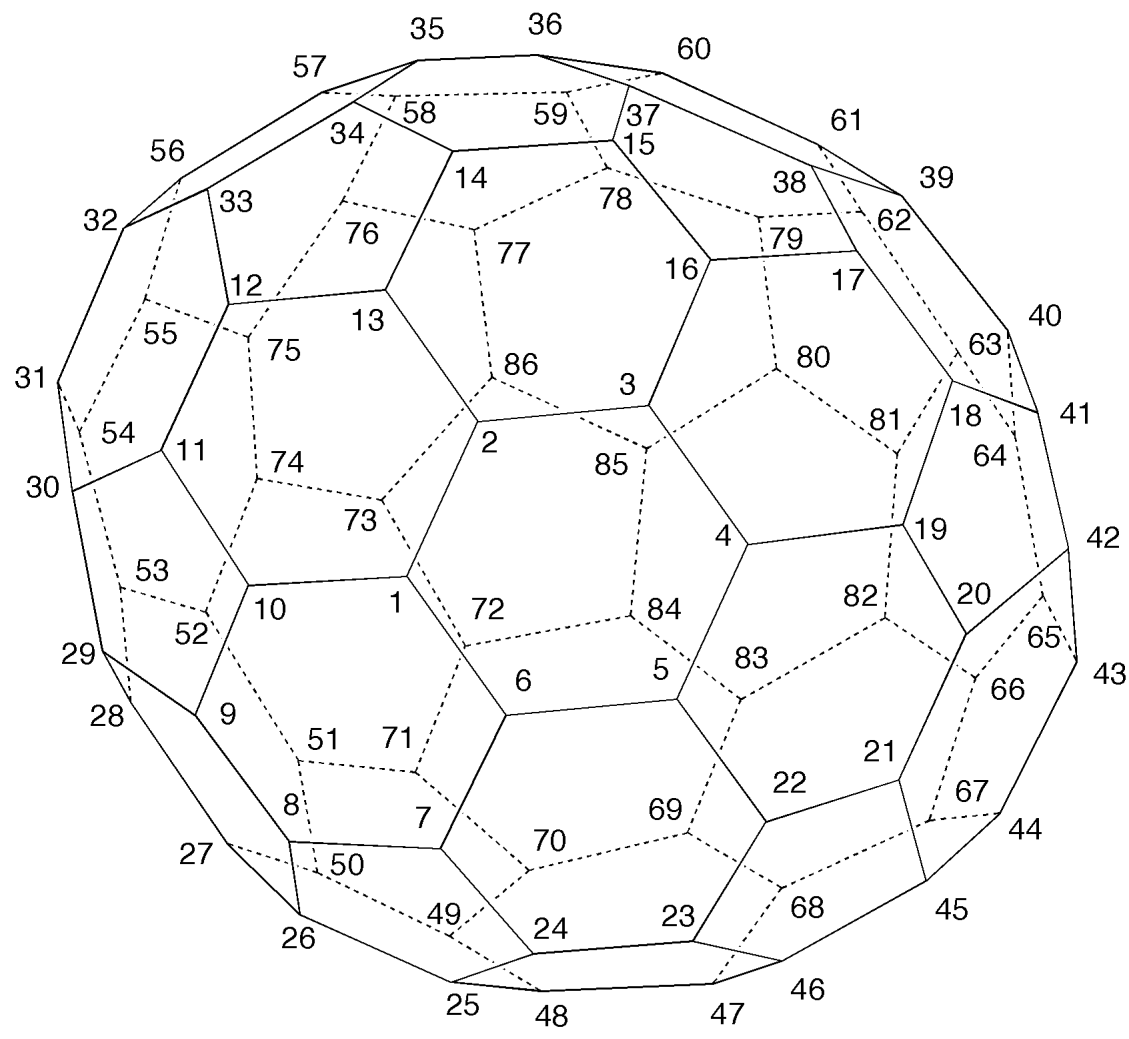

31 


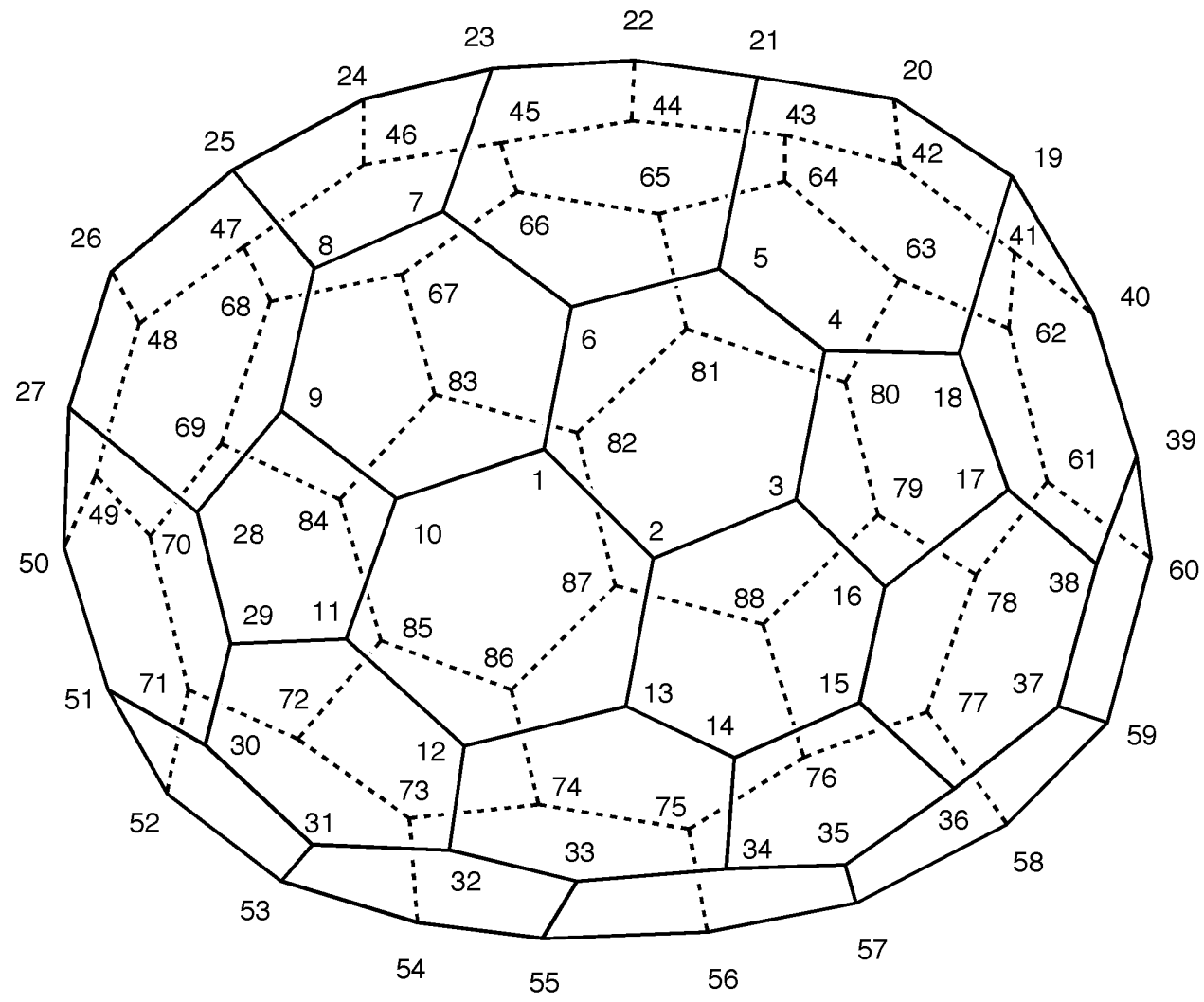




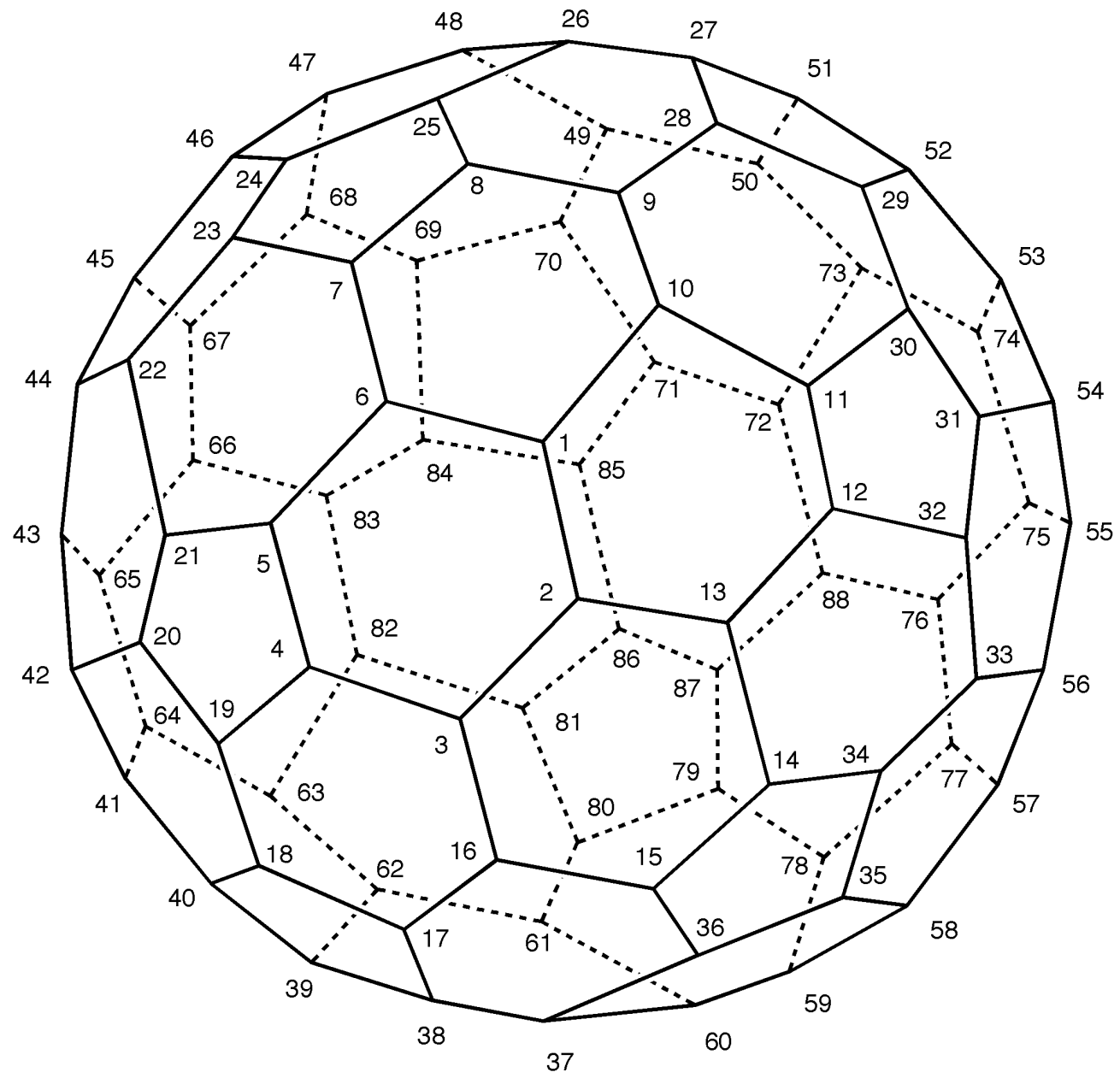




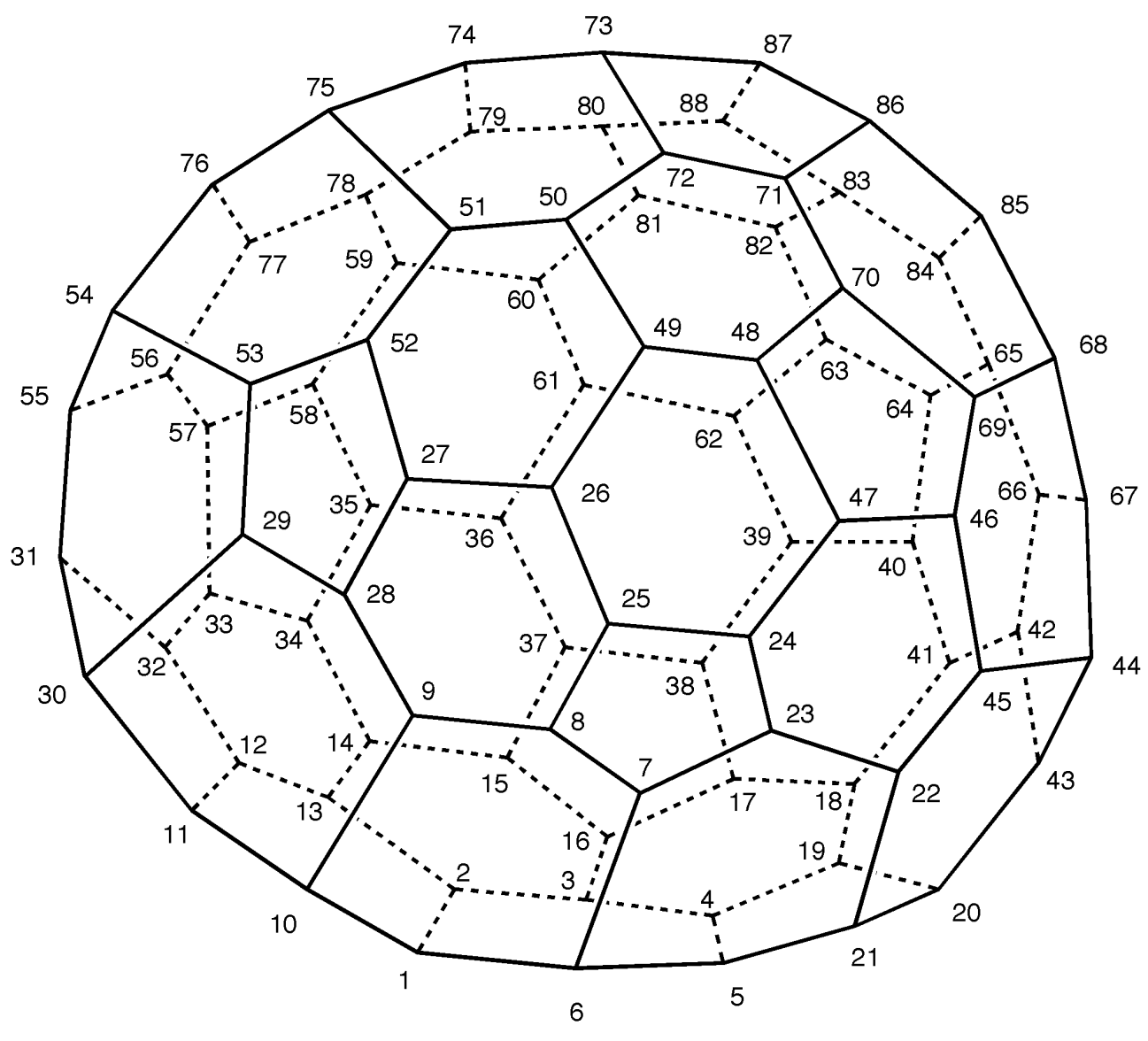




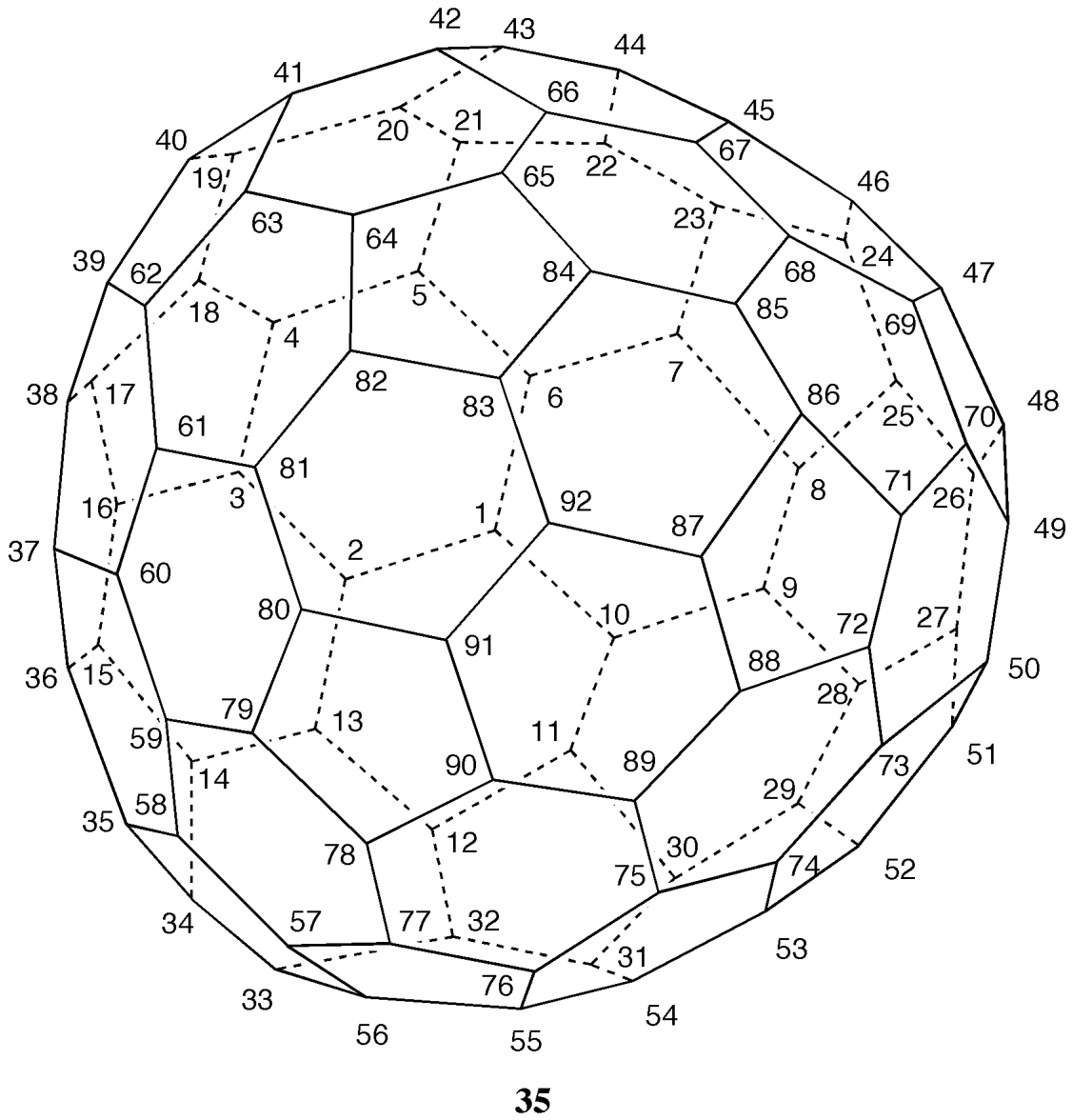

